University of Louisville

ThinkIR: The University of Louisville's Institutional Repository

$12-2007$

\title{
Soil phosphate detection and archaeology : in-stride phosphate detection and the elimination of arsenate interference to the malachite green method.
}

Laura A. DeNeve

University of Louisville

Follow this and additional works at: https://ir.library.louisville.edu/etd

\section{Recommended Citation}

DeNeve, Laura A., "Soil phosphate detection and archaeology : in-stride phosphate detection and the elimination of arsenate interference to the malachite green method." (2007). Electronic Theses and Dissertations. Paper 331.

https://doi.org/10.18297/etd/331

This Master's Thesis is brought to you for free and open access by ThinkIR: The University of Louisville's Institutional Repository. It has been accepted for inclusion in Electronic Theses and Dissertations by an authorized administrator of ThinkIR: The University of Louisville's Institutional Repository. This title appears here courtesy of the author, who has retained all other copyrights. For more information, please contact thinkir@louisville.edu. 


\title{
SOIL PHOSPHATE DETECTION AND ARCHAEOLOGY: IN-STRIDE PHOSPHATE DETECTION AND THE ELIMINATION OF ARSENATE INTERFERENCE TO THE MALACHITE GREEN METHOD
}

\author{
By \\ Laura A. DeNeve \\ B.A., Bellarmine University, 2004
}

\begin{abstract}
A Thesis
Submitted to the Faculty of the

Graduate School of the University of Louisville

in Partial Fulfillment of the Requirements

for the Degree of
\end{abstract}

Master of Science in Chemistry

Department of Chemistry

University of Louisville

Louisville, Kentucky

December 2007 


\title{
SOIL PHOSPHATE DETECTION AND ARCHAEOLOGY: IN-STRIDE PHOSPHATE DETECTION AND THE ELIMINATION OF ARSENATE INTERFERENCE TO THE MALACHITE GREEN METHOD
}

\author{
By
}

Laura A. DeNeve

B.A., Bellarmine University, 2004

A Thesis Approved on

December 2007

By the following Thesis Committee:

Thesis Director 
This thesis is dedicated to

my family,

who have supported me throughout my educational journey. 


\section{ACKNOWLEDGEMENTS}

I would like to thank my advisor, Dr. Heather Rypkema, for her endless support, guidance, and patience. I would also like to acknowledge my other committee members, Professor Yappert and Professor Rich. I would also like to thank my friends and family for the support and encouragement. I also owe a great deal of thanks to my fellow group members and friends in the department. 


\begin{abstract}
SOIL PHOSPHATE DETECTION AND ARCHAEOLOGY:

IN-STRIDE PHOSPHATE DETECTION AND THE ELIMINATION OF ARSENATE INTERFERENCE TO THE MALACHITE GREEN METHOD
\end{abstract}

\author{
Laura A. DeNeve
}

December, 2007

Archaeologists use soil analysis to detect chemicals, like phosphate, to indicate areas of anthropogenic activity. Phosphate detection is a multi-step process, which makes standard techniques time consuming.

Kinetic studies decreased the analysis time for the malachite green (MG) method of phosphate detection. The 3-minute method allows extraction and analysis to be complete in 15 minutes. Continued studies resulted in two-color spectral monitoring, which provided values instantaneously.

Arsenate $(\operatorname{As}(\mathrm{V}))$ interfers with the $M G$ method and results in overestimation of phosphate. As(V) must be reduced to non-interfering arsenite. Two As $(\mathrm{V})$ reducing agents - L-Cysteine and thiosulfate - were investigated. The thiosulfate method was suitable for field implementation with the 3-minute malachite green method. L-Cysteine is compatible with both MG time scales, but pre-reduction could not be improved beyond 20 minutes.

The 3-minute malachite green method was utilized at an archaeological site in Virginia The survey led to delineation of the site boundaries. 


\section{TABLE OF CONTENTS}

ACKNOWLEDGEMENTS ................................................................. iv

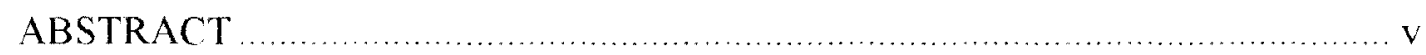

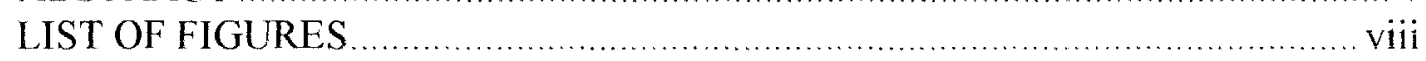

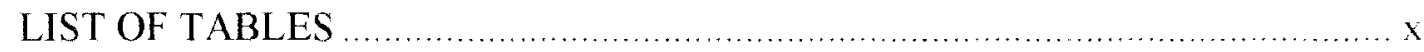

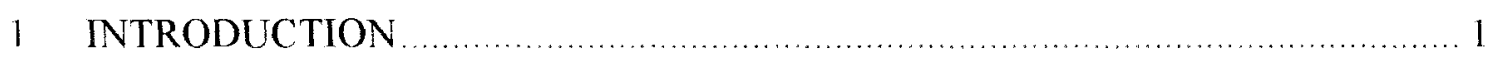

1.1 Phosphate and Archaeology ............................................................ 1

1.2 Phosphate Soil Chemistry ................................................................ 7

1.3 Phosphate Analysis .................................................................... 10

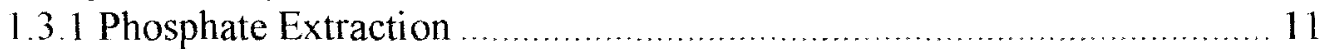

1.3.2 Phosphate Detection ............................................................. 14

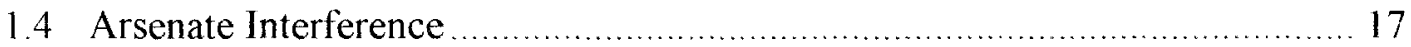

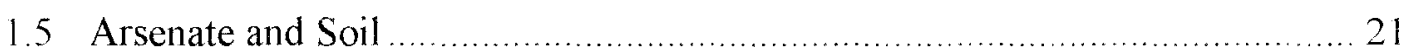

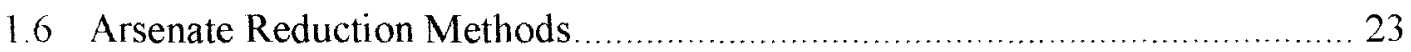

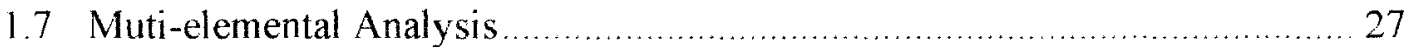

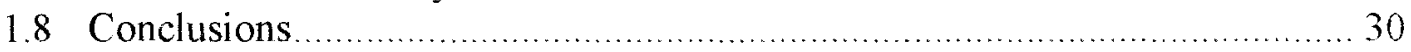

2 SOIL PREPARATION AND EXTRACTION …....................................... 37

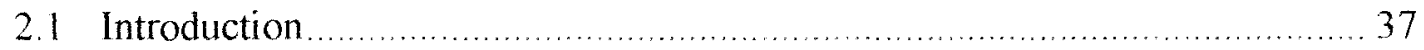

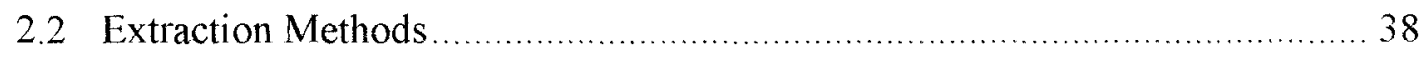

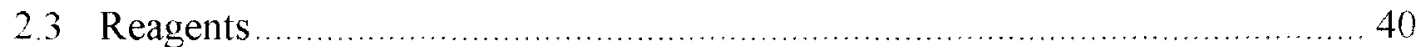

2.4 Laboratory Preparation and Extraction ............................................... 41

2.4.1 Comparison of Extraction Methods ....................................... 42

2.4.2 Mehlich 3 Reproducibility ................................................... 47

2.5 In-stride Preparation and Extraction .................................................... 48

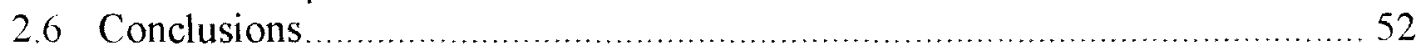

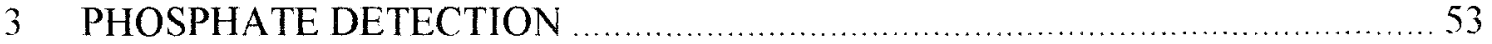

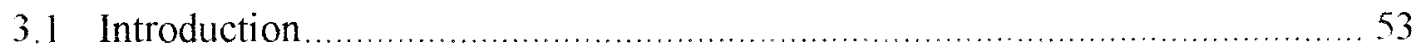

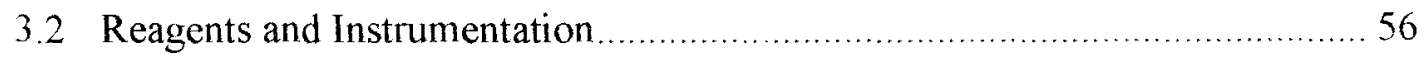

3.3 Three Minute Phosphate Analysis ....................................................... 57

3.4 Instantaneous Dual Absorbance Analysis ............................................. 63

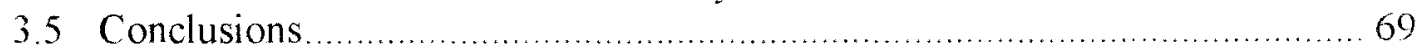

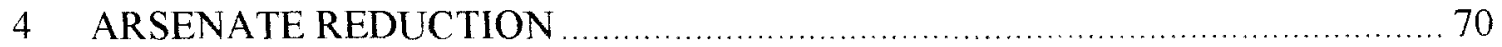

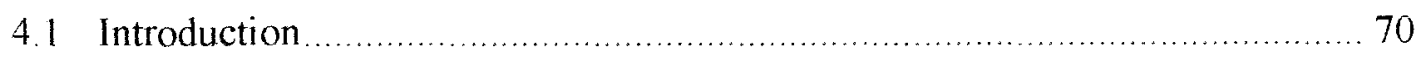

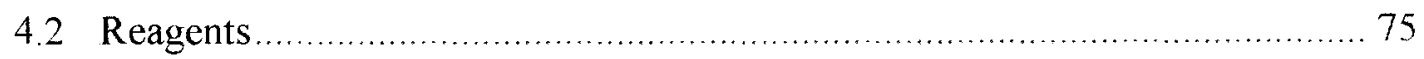

4.3 Kinetic Effects of Arsenate on Phosphate Analysis ............................... 75

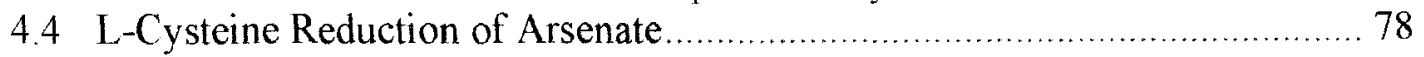

4.5 Thiosulfate Reduction of Arsenate ............................................... 82 
4.6 Comparison of Reducing Agent Efficacy ……....................................... 88

4.7 Reducing Agent Compatibility with Dual Absorbance Phosphate Analysis.... 90

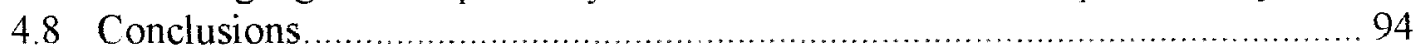

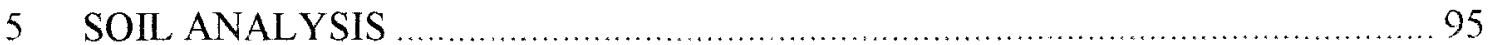

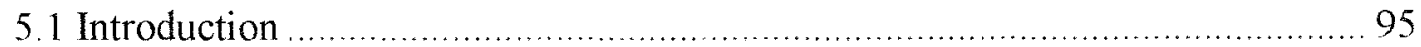

5.2 Instrumentation and Reagents …........................................................... 97

5.3 In-stride Phosphate Analysis .................................................................. 99

5.4 Additional Laboratory Soil Analysis.......................................................... 103

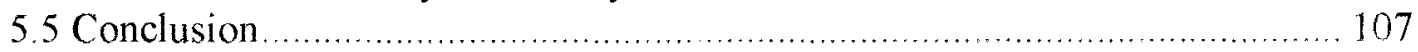

6 FUTURE DIRECTIONS AND CONCLUSIONS _......................................... 109

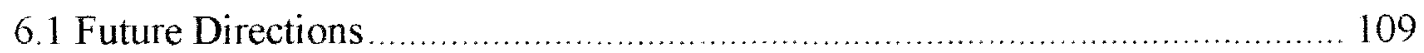

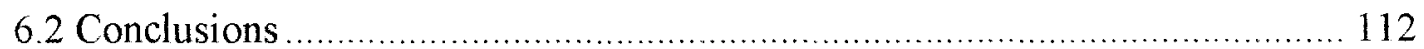

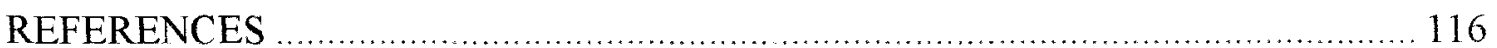

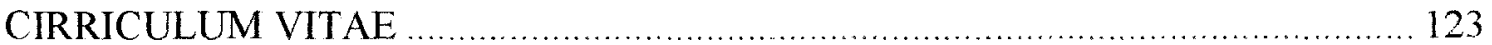




\section{LIST OF FIGURES}

Figure 1-1: Distribution of phosphorus at a domestic structure from a archaeological site in Catalyhoyuk Turkey as reported by Middleton (2004). ................................. 3

Figure 1-2: Structure of phosphate, shown in three forms......................................... 7

Figure 1-3: Structure of arsenate, shown in three forms .................................... 18 Figure 1-4 A comparison of the absorbtion spectra of phosphomolybdate and Arsenomolybdate complexes....................................................................... 19

Figure 1-5 As(V) increases the absorbance and causes inaccuracies in phosphate

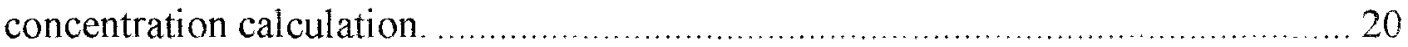

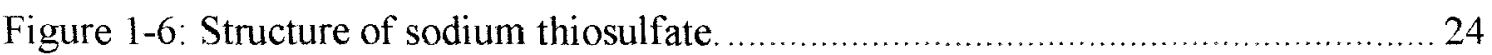

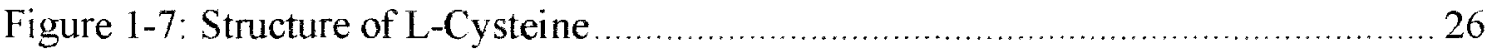

Figure 2-1. Syringe filtration of soil and extractant mixture ................................ 42

Figure 2-2. The absorbance of the malachite green and molybdophosphate complex is

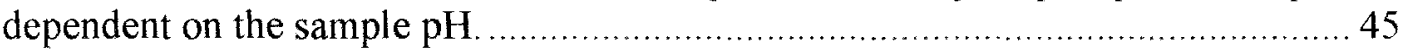

Figure 2-3. The filtrates from the different extraction methods show a wide variation in

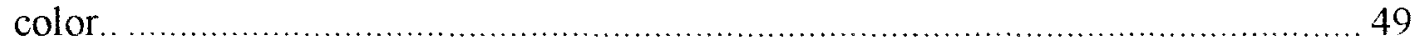

Figure 2-4. Phosphate concentrations calculated from wet and dry extractions show

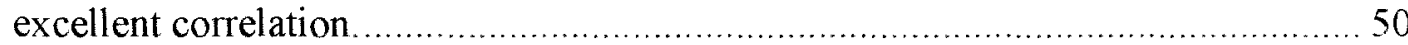

Figure 3-1: The structures of malachite green ......................................................5 54

Figure 3-2: The absorbance spectra of the green and yellow forms of malachite green. . 55

Figure 3-3: The absorbance spectrum of the premixed developing reagent ..................58

Figure 3-4: Kinetic analysis of the two methods of developing reagent addition ...........59

Figure 3-5: A comparison of 3 minute and 1 hour development ..................................62

Figure 3-6: The spectra of two different phosphate concentrations show absorbance at

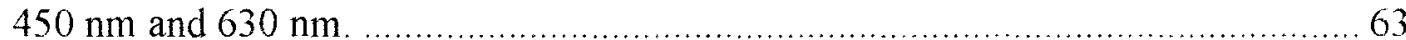

Figure 3-7: The difference in the absorbance at $473 \mathrm{~nm}$ and $630 \mathrm{~nm} \ldots \ldots \ldots \ldots \ldots \ldots \ldots \ldots \ldots . . . . . .67$

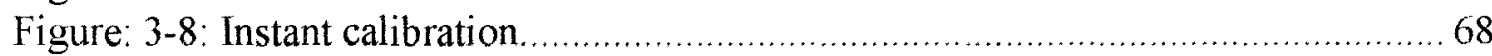

Figure 4-1: Spectra of phosphomolybdate-malachite green and arsenomolybdatemalachite green complexes .................................................................... 71

Figure 4-2; $\operatorname{As}(\mathrm{V})$ increases the absorbance and causes inaccuracies in phosphate

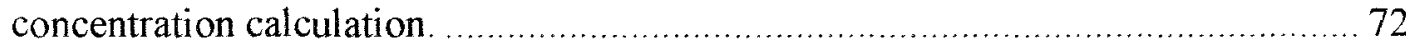

Figure 4-3: A comparison of the kinetics at $630 \mathrm{~nm}$ of $\mathrm{As}(\mathrm{V})$, phosphate, and mixed

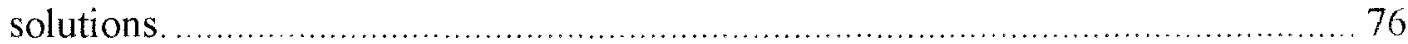

Figure 4-4: A comparison of the kinetics at $473 \mathrm{~nm}$ of $\mathrm{As}(\mathrm{V})$, phosphate, and mixed

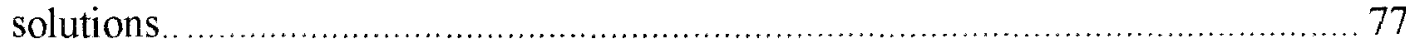

Figure 4-5: Concentration dependence of $L$-Cysteine reduction ....................... 80

Figure 4-6: Time dependence of L-Cysteine reduction. ........................................8 80

Figure 4-7: Kinetic analysis at $630 \mathrm{~nm}$ of L-Cysteine reduction................................ 81

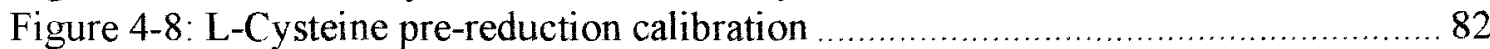

Figure 4-9: Time dependence of thiosulfate reduction .......................................... 84

Figure 4-10: Calibration made by separate addition of thiosulfate reducing reagents . . . 85 
Figure 4-11: Kinetic Analysis of Thiosulfate Pre-reduction at $630 \mathrm{~nm}$ 86

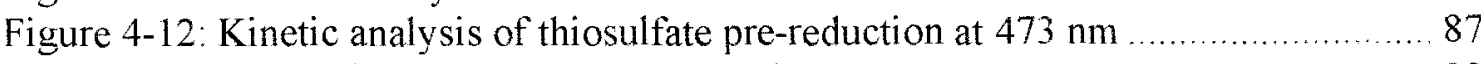

Figure 4-13: Thiosulfate pre-reduction calibration ............................................... 88

Figure 4-14: Instantaneous absorbance measurements of thiosulfate reduced solutions. 91

Figure 4-15: Instantaneous absorbance measurements of L-Cysteine solutions ............. 92

Figure 5-1: The Blue Ridge Center for Environmental Stewardship located in Loudoun Co. Virginia 96

Figure 5-2 Compact and portable UV-VIS spectrophotometer used for absorbance measurements on-site. 98

Figure 5-3: Portable toolbox used to transport all necessary items for phosphate analysis during the in-situ survey.

Figure 5-4: Spot map produced using phosphate concentrations determined from in-stride analysis.

Figure 5-5: Metal detector hits producing metal artifacts were in areas of heightened phosphate.

Figure 5-6: Phosphate concentrations determined after thiosulfate.......................... 105

Figure 5-7: Phosphate concentrations determined after L-Cysteine reduction ............... 106

Figure 6-1: Correlation between iron and phosphate concentration. 


\section{LIST OF TABLES}

Table 2-1. A Comparison of the phosphate concentration resulting from different extraction methods

Table 2-2. Average phosphate concentration resulting from three Mehlich 3 laboratory

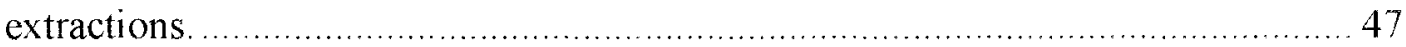

Table 2-3: Average Phosphate concentrations determined from three Mehlich3

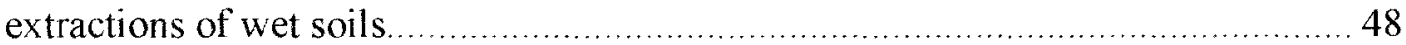

Table 4-1: Summary of arsenate reduction methods ............................................. 73

Table 4-2: A comparison of the reduction efficiency of thiosulfate and L-Cysteine ......90

Table 4-3: L-Cysteine efficacy with the instantaneous, dual absorbance method.......... 93

Table 5-1: Establishment of "high" concentrations of phosphate ............................. 100

Table 5-2: Comparison of phosphate levels determined using the commercially available $\mathrm{HACH}$ colorimeter and the malachite green method. 104

Table 5-3: Phosphate concentrations determined using the instantaneous, dual absorbance

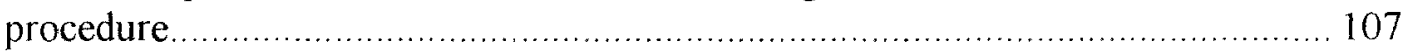

Table 6-1: Iron concentration in 10 soil samples from Loudon County, Virginia ....... 110 
INTRODUCTION

\subsection{Phosphate and Archaeology}

The field of archaeology has benefited from chemistry in many ways. For example, radiocarbon dating has made a profound impact on archaeology by increasing the accuracy of chronological analysis. Additional analytical techniques that have crossed into the field of archaeology include laser induced breakdown spectroscopy, Raman spectroscopy, and inductively coupled plasma absorption emission spectroscopy These techniques have been used to characterize historical objects, which provide valuable information pertaining to the materials and technology of the period.

The above techniques are all used after the excavation of the artifact. While some historic sites are easily located, it is often difficult to determine the layout of settlements, intrastate usage and settlement hierarchy. For settlements abandoned within more recent history, this task is aided by historic documents such as deeds and wills, but for many societies these documents are no longer available. Other solutions utilize remote sensing methods to aid in the determination of boundary lines. For example, aerial photography and high spatial resolution satellite imagery are used to map potential ancient settlements. Infrared and thermal imaging are used to detect buried remains. However, these four techniques cannot be utilized in areas with dense vegetation. In heavily forested areas, ground survey methods are employed. Ground penetrating radar, magnetic prospection, 
and shovel tests are conducted by surveying areas on foot. As sites are often many hectares, the tests are conducted at $30-50 \mathrm{~m}$ intervals. In the event the test is positive and locates an artifact additional tests are conducted in the surrounding area. These tests only provide data on the area sampled and it is highly probable that historical objects are missed.

Ground surveys have been improved by soil analysis. Many anthropogenic activities deposit elements in soil. The majority of the elements left behind are rapidly depleted from the soil or the elements are mobile and do not remain in the original location. Phosphorus binds rapidly with $\mathrm{Fe}, \mathrm{Al}$, or $\mathrm{Ca}$ in soil and becomes relatively immobile. Prolonged occupation of a settlement causes phosphorus to accumulate, and as a result areas of human occupation show large concentrations of phosphorus when compared to native phosphorus. For this reason, large archaeological sites boundaries can be delineated from the surrounding irrelevant areas.

Sarris at el. (2004) used soil phosphorus detection as well as traditional techniques, such as shovel testing and magnetic detection, during survey of Veszto-Bikeri in Southeastern Hungary. As a result, the layout and organization of a dispersed agricultural settlement was reconstructed. The phosphorus analysis indicated the site was composed of 10 or 12 trenched compounds and structures. Additionally Sarris et al. were able to determine the location of kilns, ovens, hearths, and a midden.

Phosphorus analysis is also extremely useful at sites where the boundaries and layout of the settlement have already been determined. Middelton (2004) studied the phosphorus distribution within an archaeological site in Catalhoyuk, Turkey. As seen in Figure 1-1, there was a large variation in the amount of phosphorus within the remains of 
a building. The high concentration of phosphorus indicates the area was once used as an interior hearth. The phosphorus accumulated as a result of repeated burnings of organic material.

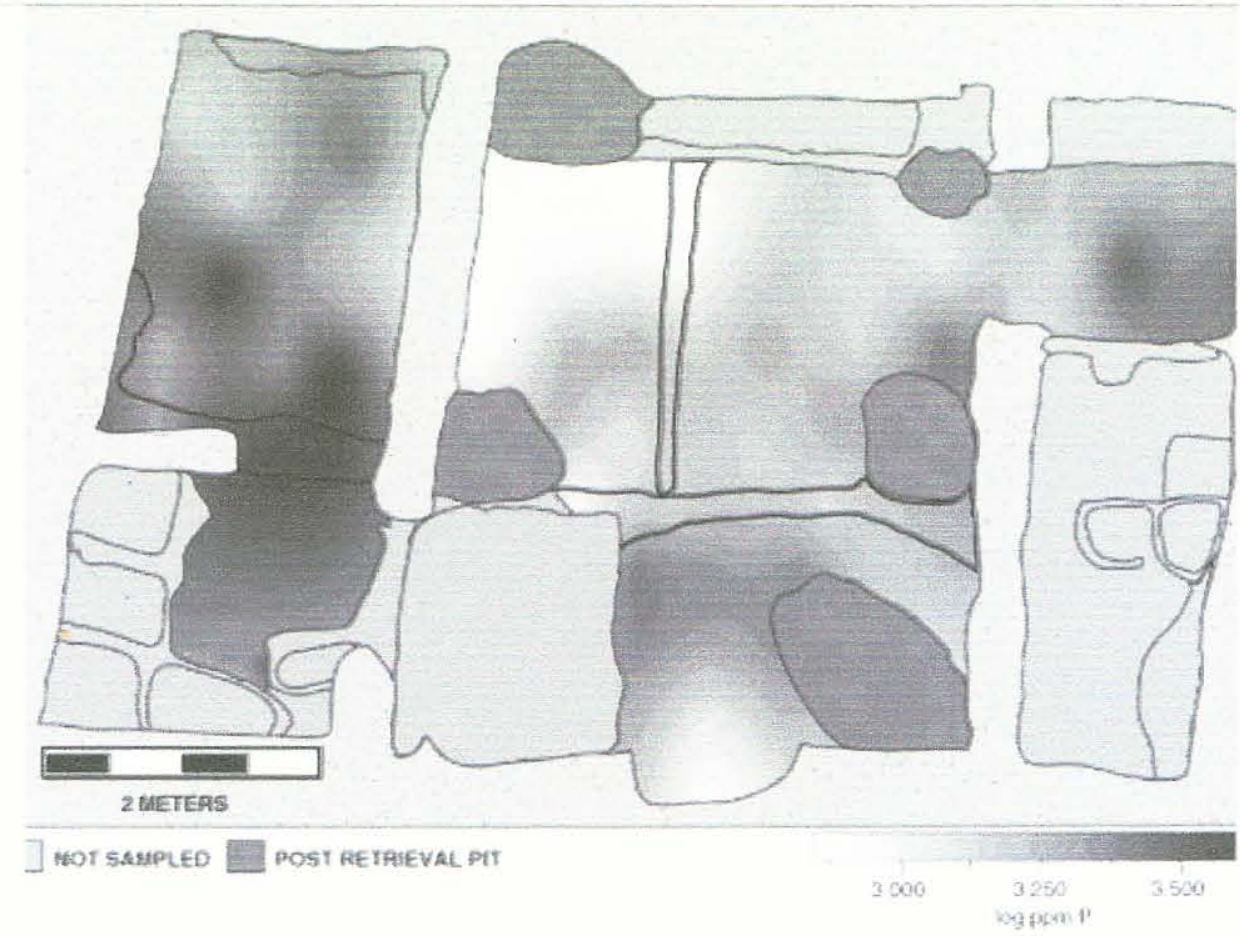

Figure 1-1: Distribution of phosphorus at a domestic structure from an archaeological site in Catalyhoyuk Turkey as reported by Middleton (2004).

Phosphorus analysis can be used on both pre-agricultural and agricultural societies. There are several anthropogenic activities all societies have in common-such as meal preparation, waste disposal and phosphorus enrichment around burial sites. Other activities are specific to a particular culture or settlement type-such as religious ceremonies and agricultural activities. Each activity increases the phosphorus in the area the activity occurred creating a "hot spot" of phosphorus.

Hearths and kitchen middens are hot spots created by meal activities. The burning of organic tissue from both plants and animals, as well as the ash from the fire, 
enriches the phosphorus concentration in the area surrounding the hearth. Sjöberg (1976) explains a careful study of the hearth area is useful for two reasons. First, information gained allows archaeologists to infer the use of the rooms containing the hearth. Low amounts of phosphate suggested it was used solely for warming and higher levels of phosphorus indicated the hearth was used for food preparation. Second, analysis of the levels of phosphorus in the hearth may also give evidence to the diet of the people since animal food products result in greater deposition of phosphorus than plant products.

It is important to determine the location of the kitchen midden since it is the area where many domestic items, such as pots and utensils, were also discarded. Waste from meals was also deposited in the kitchen middens. Therefore as a result of the large amount of decaying meat, fish and plant refuse, the area surrounding the midden has elevated level of phosphorus

Burial sites are rich in phosphorus because phosphorus is a main component in DNA and RNA. 1966. Bóna's excavated a $6^{\text {th }}$ - century grave. Control soil samples and soils immediately surrounding the grave were analyzed to determine the concentration of phosphate. When the levels of phosphate were compared, it was easy to see the soil surrounding the grave had significantly higher levels of phosphorus. In fact, the high levels of phosphorus created an outline of the skeleton (Duma, 1972).

Agricultural societies have large areas with high levels of phosphate. Barns and stables accumulate phosphorus from the animal waste. Similarly, well traveled herding paths and animal grazing areas have elevated phosphorus. Fertilizer used in crop fields enriched soil phosphorus because it was created from guano, animal products and human waste. Animal pens and crop fields can be separated from housing structures because 
agriculture sites cover more area and have higher levels of phosphorus. Craddock et al. (1985) excavated a settlement in Fengate, Petersborough, known as Cat's Water, containing many huts that were randomly distributed. It was important to distinguish the huts that were once used for human occupation from those used for animals, which are less likely to contain historical objects. This task was accomplished through soil analysis and Craddock and coworkers were able to separate the animal pens from the housing sites.

Depending upon the society, religious rituals may also lead to the accumulation of phosphorus. Religious altars used for sacrifices have higher levels of phosphorus as a result of decaying animal tissue and bones. It should be noted that these areas only have a slight enrichment of phosphorus since rituals are less likely to be preformed daily. Sánchez et al. (1996) discovered a ram skull in an area of high phosphorus. After considering the location of the skull, the level of phosphate, and the culture of this prehistoric settlement, the group determined the ram skull was likely used in a fertility ritual. The ash accumulated from the burning of incense also enriches soil phosphorus.

After the phosphate levels have been determined and the artifacts have been excavated, several archaeologists have seen a strong correlation between the phosphorus level and the location of the artifact. Sánchez at el. (1996) excavated a site in the southeastern quarter of the Iberian Peninsula that was approximately 40 ha. Several objects of cultural significance were located in areas with elevated levels of phosphorus. The materials found included ceramic pots, bowls, platters, dishes, flint and potsherds.

Craddock et al. (1985) also found a correlation between the location of artifacts and the level of phosphorus during the excavation of an Iron Age settlement in Maxey, 
Cambridgeshire. The twelve-acre site was known to have archaeological significance; however at the time of the excavation it was a working plough field. The eastern portion of the field had higher levels of phosphorus than the western portion. The west fields did not yield any significant archaeological finds. However, the eastern portion, with the higher phosphorus level, contained pottery shards and bones. In both Craddock and Sanchez' excavations, there was a clear correlation between phosphorus and artifacts, which validates the use of phosphate soil analysis.

Phosphorus detection becomes most useful when surveying potential sites with rough terrain. Bjelajac et al. (1996) demonstrated the effectiveness of soil phosphate analysis when attempting to determine the boundaries of an archaeological site in Sunol Valley, California. A visual survey of the site was nearly impossible since the site was analyzed during a period of dense vegetation and surface artifacts could not be seen. The group sampled 33 soils that represented soils considered on-site and off-site. The phosphate levels were compared and Bejelajac et al. discerned the boundaries of the site, facilitating a more effective search for artifacts.

Soil phosphorus analysis is an important archaeological tool capable of both delineating large archaeological sites and aiding the determination of intra site usage. However, the quantitative soil analysis methods used in the examples above are lengthy and require a familiarity with the chemical principles involved. Therefore, analysis often occurs in a laboratory setting at a museum or university. The benefits of soil analysis are diminished by the time requirements of shipping soil to a new location for testing. The length of quantitative phosphorus detection methods must be shortened and made portable in order to be incorporated into large scale archaeological surveys. 


\subsection{Phosphate Soil Chemistry}

Phosphorus soil chemistry is rich and complex, but a basic knowledge of the subject is imperative to understanding why phosphorus is more significant than other elements for archaeological analysis. Additionally, phosphorus soil chemistry has a large effect on the selection of suitable extraction and analysis methods.

Phosphorus has a strong affinity for oxygen and accordingly, in soil, phosphorus is found as orthophosphate. There are three forms of orthophosphate: $\mathrm{H}_{2} \mathrm{PO}_{4}^{-}, \mathrm{HPO}_{4}{ }^{2-}$, and $\mathrm{PO}_{4}{ }^{3-}$ (Figure 1-2). Collectively, in the context of archaeological discussions, all three forms are generalized as phosphate
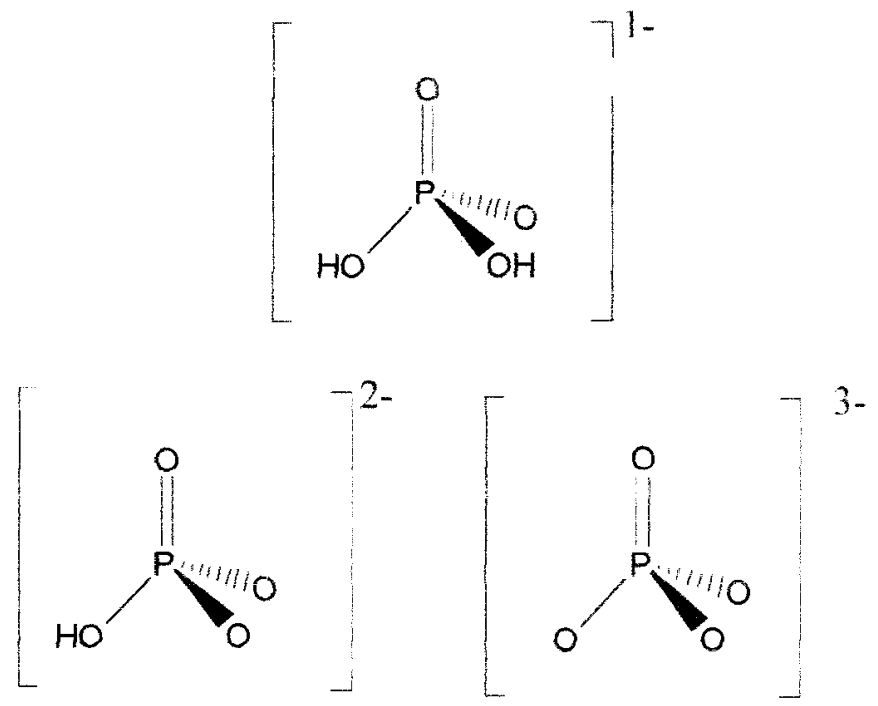

Figure 1-2: Structure of phosphate, shown in three forms

Phosphate is found as a precipitate in soil. It is either adsorbed to the surface of the soil particle or occluded within the soil particle. If the phosphate is occluded, it is either physically incorporated or chemically entrapped in the soil particle. Accordingly, occluded phosphate is more difficult to extract. 
Phosphate can be found in soil in both inorganic and organic forms. Organic forms are linked to an ester, whereas inorganic forms are bound to metallic cations. Archaeologists are typically only interested in inorganic phosphate or total phosphate (Bjelajac, 1996). Organic phosphate on its own is not archaeologically significant since it accumulate in the soil as the result of recently decomposed material. Over time, organic phosphate is converted into the inorganic form through mineralization. Microorganisms control the mineralization of organic phosphate. The moisture content of the soil affects the extent of microorganism activity and therefore, the extent of mineralization depends on the season (Sanchez et al. 1996; Schlezinger, 2000.).

Inorganic phosphate readily binds to $\mathrm{Fe}^{3+}, \mathrm{Ca}^{2+}$, or $\mathrm{Al}^{3+}$ depending on the $\mathrm{pH}$ of the soil. Highly acidic soils ( $\mathrm{pH}$ below 5 ) contain a mixture of phosphates bound to $\mathrm{Al}^{3}$ and $\mathrm{Fe}^{3+}$. Basic soils with a $\mathrm{pH}$ above 8 have phosphates bound to $\mathrm{Ca}^{2}$. Phosphate ions in soils with moderate $\mathrm{pH}$ between 5-8 are bound to all three cations (Lillios, 1992; Sinaj et al. 2002). Changes in the pH of soil affect the fixation of phosphorus. For example, if the $\mathrm{pH}$ of a basic soil decreases, the phosphate bound to $\mathrm{Ca}^{2+}$ is released and a portion of the phosphate may be lost to plants. The remaining phosphate binds to $\mathrm{Al}^{3+}$ and $\mathrm{Fe}^{3+}$ and creates new insoluble compounds (Schlezinger, 2000). The $\mathrm{pH}$ of the soil affects the choice of extracting reagent. Weakly acidic extracting reagents are capable of soluabilizing the bonds between phosphorus and $\mathrm{Ca}^{2}, \mathrm{Al}^{3+}$ and to a lesser extend $\mathrm{Fe}^{3}$. These extracting reagents are used for acidic soils. Strong acids, such as $\mathrm{HClO}_{4}$, are more aptly suited for alkaline soils. Extracting reagents with a slightly basic $\mathrm{pH}$ are also used (Lillios, 1992). 
Inorganic phosphate is classified by the strength of the bond formed with the soil particle. Phosphate is considered available, active or occluded. Available phosphate has weak bonds and is considered mobile. It is easily transformed to organic phosphate and highly susceptible to dissolution and desorption. As a result, it is readily available to be taken up by plants. Typically, the loss of $\mathrm{P}$ to plants is minimal and does not affect archaeological analysis. The available forms of phosphate are $\mathrm{H}_{2} \mathrm{PO}^{-}$and $\mathrm{HPO}_{4}^{2-}$ since these forms have lower bonding energy and are more soluble. This type of phosphate is easily extracted from the soil using mild reagents; however, it represents only a small portion of the total phosphate in soil.

Active phosphate has moderately strong bonds with the soil particles. In this case, the phosphate only undergoes transformation to organic phosphate, dissolution and desorption after prolonged exposure to microorganisms and moisture during rainy season. Active phosphate is the prevalent fraction of phosphate at an archaeological site. The extracting reagents used are stronger than those used on available phosphate because of the increased bond strength.

Occluded phosphate is strongly bound to the soil particles. Thus, it is not readily transformed, desorpt and it is not susceptible to dissolution. It is virtually immobile in soil, except in a few select soil environments. Occluded phosphate is an excellent indicator of human habitation because it does not move vertically throughout the soil profile and is not lost to plant sorption. As a result of the high degree of stability of occluded soil phosphorus, archaeological sites dating back to the late Bronze Age (15001000 B.C.E) can be studied (White, 1978). For example, Craddock et al. (1997) were able to determine Bronze Age settlement areas by analyzing the phosphate concentration 
in soil sampled at a depth of $70 \mathrm{~cm}(27.5$ inches $)$. However, it should be noted that occluded phosphate is difficult to extract from the soil. Strong acid solutions and highly concentrated reagents must be used.

Phosphate cycles throughout the environment relatively slowly on a geological timescale and the removal of appreciable amounts of phosphate requires millions of years. Thus, natural processes do not conceal phosphate. However, the distribution can be altered through natural processes. Water erosion leads the relocation of small concentrations of phosphate (Schlezinger, 2000). Available and active phosphates are more likely to be affected by water erosion than occluded phosphate. However, the majority of phosphate lost to water erosion is from the topsoil (2-5 inches). Archaeological soils are 6-12 inches deep and these deeper layers are not greatly affected.

The distribution of phosphate is also changed by current anthropogenic activities. Phosphate prospection is difficult in actively inhabited areas because modern farming, animal husbandry, terrain modification and waste production interfere with accurate analysis of the past activities. Interference from recent changes to the phosphorus in soil can be avoided by obtaining soil samples from deeper soil horizons.

\subsection{Phosphate Analysis}

The process of determining the amount of phosphate in soil is completed in three steps. First, the soil must be removed from the ground. Soil samples are not collected from the topsoil. Rather, soil is collected from deeper layers of the soil horizon in order to prevent interference from recent anthropogenic activities. The depth at which the soil is sampled depends on the time period of the settlement. Either the A soil horizon or the 
B horizon (6 inch and 12 inch deep, respectively) is sampled to obtain phosphate concentrations for settlements abandoned within the last several hundred years. Soil is taken from a deeper location of the soil profile for archaeological sites dating back to more ancient times.

After the soil is removed from the earth, it must be ground into fine particles and filtered to remove organic debris. At this point, the phosphate is extracted from the soil using chemical reagents to break the bonds between the phosphorus and the soil particulate. Over 50 phosphate extraction methods have been published in soil science literature (Holliday and Gartner, 2007). The majority of the methods are used by soil scientists in the agricultural field. However several of the methods have been used for archaeological purposes

The final step in phosphate determination is the analysis of the extract. There are multiple methods of phosphate analysis and most were designed for agricultural purposes. Commercially available analysis procedures used commonly for phosphate detection in water have also been employed for phosphate prospection of archaeological sites.

\subsubsection{Phosphate Extraction}

The many phosphate extraction methods can be separated into groups according to the type of phosphorus removed from the soil. There are three groups of soil extraction methods that are relevant to archaeology:

1. Acid digestion for total phosphorus analysis

2. Fractionation extractions, which separate the individual types of inorganic phosphate 


\section{Extraction methods for available phosphorus}

Acid digestion of soil extracts all forms of inorganic and organic phosphorus. Total phosphate is extracted using either a single strong acid, such as $\mathrm{HClO}_{4}$, or mixture of acids. The choice of acid or acids is dependent on the type of soil. For on-site extractions, $2 \mathrm{~N} \mathrm{HCl}$ has been used. However, to ensure complete digestion the samples must be heated for one hour in a steam bath. (Craddock et al. 1985). Instead of heat, other researchers used ultra sonic baths or centrifuges to ensure complete digestion (Sanchez et al. 1996).

Some archaeologists assert that methods providing information on total phosphate are poor indicators of prior human habitation (Terry et al. 2000). The different chemical forms of phosphate must be differentiated. In order to separate native phosphate from anthropogenic phosphate fractionation methods were developed. For example, Eidt and Woods (1977) proposed a fractionation method for the analysis of soil phosphate that requires seven extractions and four different colorimetry methods for the analysis of total phosphate. The first extraction requires 12 hours of shaking and the last extraction requires 4 hours of shaking. Two of the extractions require the sample be heated in a hot water bath. Although some archaeologists, such as Lillos (1992), claimed to have success using the Edit method, fractionation methods are not widely received throughout the archaeological community. Some experts argue that the method fails to account for the varying properties of different soil types (Terry et al. 2000). Others have argued that fractionation methods are "the most complex methods" used in archaeology (Sanchez et al. 1996). Therefore the method is ineffectual for large sites. 
A variety of methods are available for the extraction of available phosphorus These methods are commonly used by agricultural soil scientist and many of the methods were designed for in-field analysis. Many of the available phosphorus extraction methods are designed to accommodate a specific soil pH. Bray P1, Bray P2, and Mehlich1 methods are the most common extracting methods for acidic soils. The Bray method utilizes dilute ammonium fluoride and $\mathrm{HCl}$ and the Mehlich1 method utilizes a mixture of dilute $\mathrm{HCl}$ and $\mathrm{H}_{2} \mathrm{SO}_{4}$. The Olsen method and Ammonium bicarbonateDTPA, are used on basic soils. (Jones, 2001; Olsen and Sommers, 1982).

Two extracting reagents that have a larger soil $\mathrm{pH}$ range are the Mehlich3 method and $\mathrm{CaCl}_{2}$ method. The $\mathrm{CaCl}_{2}$ method requires 2 hours of shaking and large reagent volumes. Mehlich3 however, is well suited for fieldwork as it requires a minimal 5 minutes of shaking. In order to span a broader $\mathrm{pH}$ range, this reagent contains $\mathrm{HNO}_{3}$, which extracts $\mathrm{Ca}^{+2}$ bound phosphate, and $\mathrm{NH}_{4} \mathrm{~F}$, which extracts both $\mathrm{Fe}^{3+}$ and $\mathrm{Al}^{3+}$ bound phosphates. This method also extracts $\mathrm{Ca}^{2+}, \mathrm{Mg}^{2+}, \mathrm{Na}^{+}$, and $\mathrm{K}^{+}$.

There are several requirements that must be met in order to move phosphate analysis from the lab and into the field. Archaeological sites often cover large areas and produce many soil samples. Therefore, to be effective, analysis methods used in situ should be rapid and inexpensive. The method should require few reagents and manageable instrumentation since sites are typically in remote locations. Waste products should be limited and non-hazardous in order to prevent contamination of the environment. Finally, the extraction method should not require a great deal of technical skill, as the investigator is not always a trained chemist. 
The majority of the methods discussed above are inappropriate for in-stride analysis. The chemical digestion methods require strong and dangerous reagents, such as perchloric acid, and are only suitable for laboratory work. Edit's fractionation method is far too lengthy and requires knowledge of laboratory techniques. The only methods which meet the requirements for in-stride analysis are those designed to extract the available phosphorus.

There is a high degree of variability in the amount of phosphorus extracted using the available phosphorus extraction methods. For example, in acidic and neutral soils Mehlich3 phosphorus is 4\% more than Bray P1 phosphorus (Mehlich, 1984). Mehlich3 phosphorus in calcareous soils is notably higher than Olsen phosphorus. It has been reported to extract up to $12 \%$ more phosphorus than the Olsen method (Maiti, 2007).

The established successes of Mehlich3, combined with its versatility, make it an excellent choice for field archaeological work. This method is compatible with many methods of phosphate analysis. Therefore, Mehlich3 extracting reagent was chosen as the extraction method to be used for all soil samples subjected to phosphate analysis. The efficiency of the Mehlich3 method is limited by the preparation procedure. Chapter 2 examines possible adaptations to the physical soil preparation and the effectiveness of the Mehlich3 method for in-stride analysis.

\subsubsection{Phosphate Detection}

Multiple methods are used to determine the amount of phosphate extracted from the soil sample. The majority of the analysis is performed in a laboratory setting; however, a few methods are available for in situ analysis. One of the simplest techniques 
used in the field is known as the ring test or a spot test. Soil is placed on a filter and a few drops of acid extracting solution are applied. A color-developing reagent is added next and then the intensity of the spot is examined (Jones, 2001). The method requires the researcher to relate the color characteristics of the ring to the amount of phosphate in the soil. This method has several drawbacks. Difficulties arise due to differing color perceptions, which leads to inaccuracies. It has been suggested that ring tests are ineffectual in areas of high phosphate concentrations (Terry et al. 2000). Ring tests are also limited to qualitative data and the results change based on sample amount, which results in diminished reproducibility. These tests cannot produce gradated maps (which allow areas of high phosphate to be rapidly identified) to be used during interpretation. However, it should be noted that this method is inexpensive, rapid and easily preformed.

Commercially available kits for in-stride phosphate detection are also used (Terry et al. 2000). These kits, such as the $\mathrm{HACH}$ DR/800 portable colorimeter, use colorimetric methods of detection and provide quantitative data. Most kits are intended for water analysis; however, the HACH colorimeter has been employed for soil analysis. The phosphate is extracted from the soil using a method for the extraction of available phosphorus. Then a packet of a powdered developing reagent is added to the extract, which in the case of $\mathrm{HACH}$ colorimeter is similar to those used in the ascorbic acid method. After 10 minutes, the absorbance is obtained and related to the concentration of phosphorus. Some researchers dispute the accuracy of the HACH kits after finding the available phosphate concentration of uncontaminated water to be an order of magnitude higher than the total phosphorus in the sample (Stauffer, 1983). 
Spectrophotometric methods are also used to obtain quantitative results.

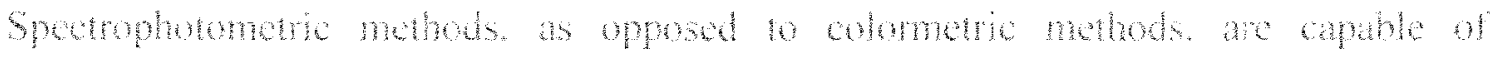
mombring multiple wavelogths simulaneously rater than determing the absolute absoption at one partoular watelength of the dye on colored compond. Spectrophotometric methods are more versatile than colormetric methods. In general, these methods rely on the formation of a complex between phosphate and molybdate. The phosphomolybdate complex then binds with a basic dye in an acidic environment. Commonly used dyes include malachite green, rhodamine $B$, and methyl green (Altmann et al. 1971; Baykov et al. 1988; D'Angelo et al. 2001; Van Veldhoven and Mannaerts, 1987). The dyes bind to the phophomolybate comples and then the twe dyes are rediued to a colones form. Sobsequenty, absomtion is due exclusively 10 phosphate bound dy. Another popular spectrophotometric method is the Murphy Riley method, also known as the ascorbic acid method. This method does not rely on basic dyes to produce color in the presence of phosphate. Rather, a phosphomolybdate complex is produced using ammonium molybdate and then reduced using ascorbic acid. Substituting vanadomolybdate for ammonium molybdate forms a more stable phosphomolybdate complex (Olsen and Sommers, 1982).

The two most widely used spectrophotometric methods are the Murphy Riley and the malachite green method. The Murphy Riley method was originally the preferred method for phosphate analysis. However in recent years the malachite green method has gained popularity, because it is more sensitive. Ohno and Zibilske (1991) state that the malachite green method is approximately 3.3 times more sensitive than the Murphy Riley method. The increase in sensitivity is the result of the higher absorption coefficient of the 
malachite green-phosphomolybdate complex when compared to the reduced phosphomolybdate complex. Furthermore, VanVeldhoven and Mannaerts (1987) state that the malachite green reagents can be stable for over a year. The robustness of this reagent is ideal for sites in remote locations where reagent preparation is difficult. Murphy Riley reagents must be made fresh daily.

After examining the advantages and disadvantages of each method, it is clear that the malachite green method of phosphate analysis possess great potential for in situ work. The method has high sensitivity and the absorbance can be determined using a portable spectrometer. Published studies state the malachite green method requires one hour for full development, making the method too lengthy for rapid analysis of multiple samples. The time dependence of the development process must be reduced in order to enhance the effectiveness of malachite green as an in-stride method. Chapter 3 discusses studies

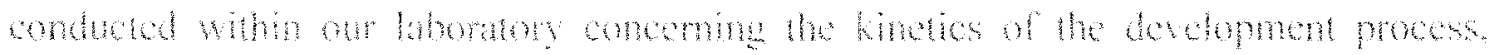

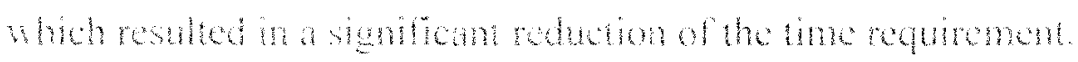

\subsection{Arsenate Interference}

Possible interferences to the malachite green method of development were examined. Iron, silica, and arsenate $(\mathrm{As}(\mathrm{V}))$ are components of soil that are capable of interfering with malachite green analysis (Linge and Oldham, 2001; Matsubara et al. 1987; Matsubara et al. 1993; Van Veldhoven and Mannaerts, 1987). Arsenate is considered a chemical analogue of phosphate. The structure of arsenate (shown in Figure 1-3) is similar to phosphate because both anions have a similar structure, symmetry and identical charge. 

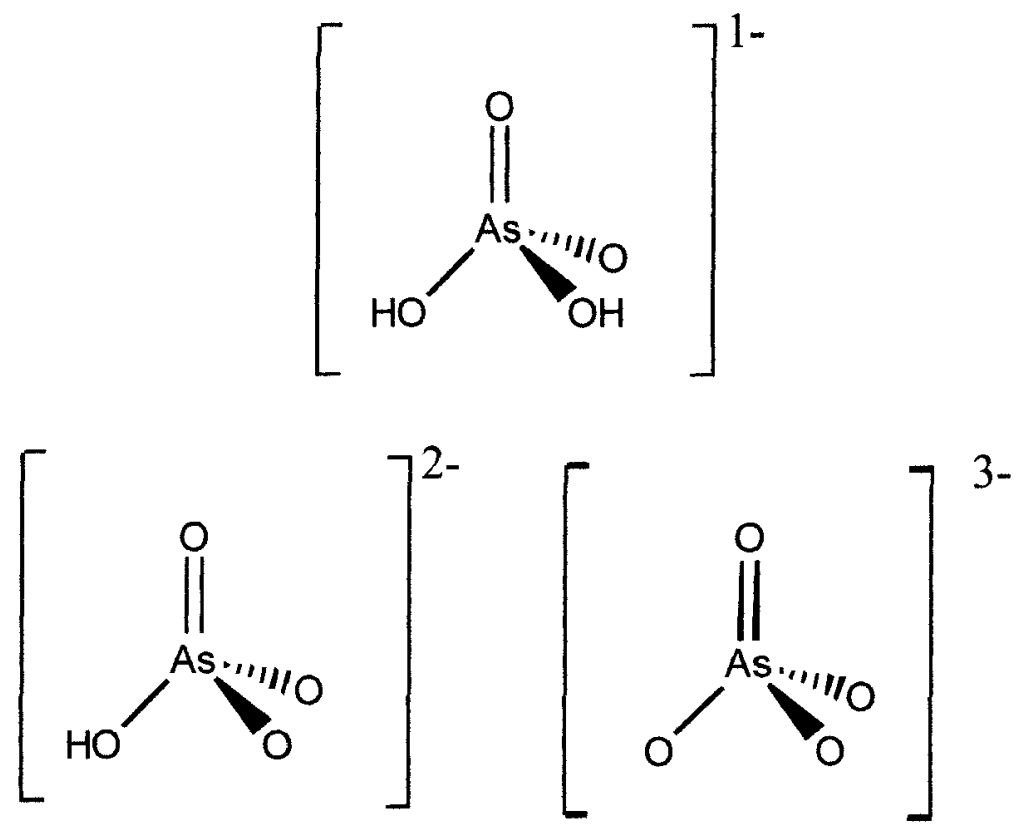

Figure 1-3: Structure of arsenate, shown in three forms.

During the malachite green phosphate detection method, $A s(V)$ complexes with molybdenum and malachite green. The interference causes an over estimation of the concentration of phosphate because both complexes are indistinguishable at the maximum absorbance of $630 \mathrm{~nm}$. (Figure 1-4) 


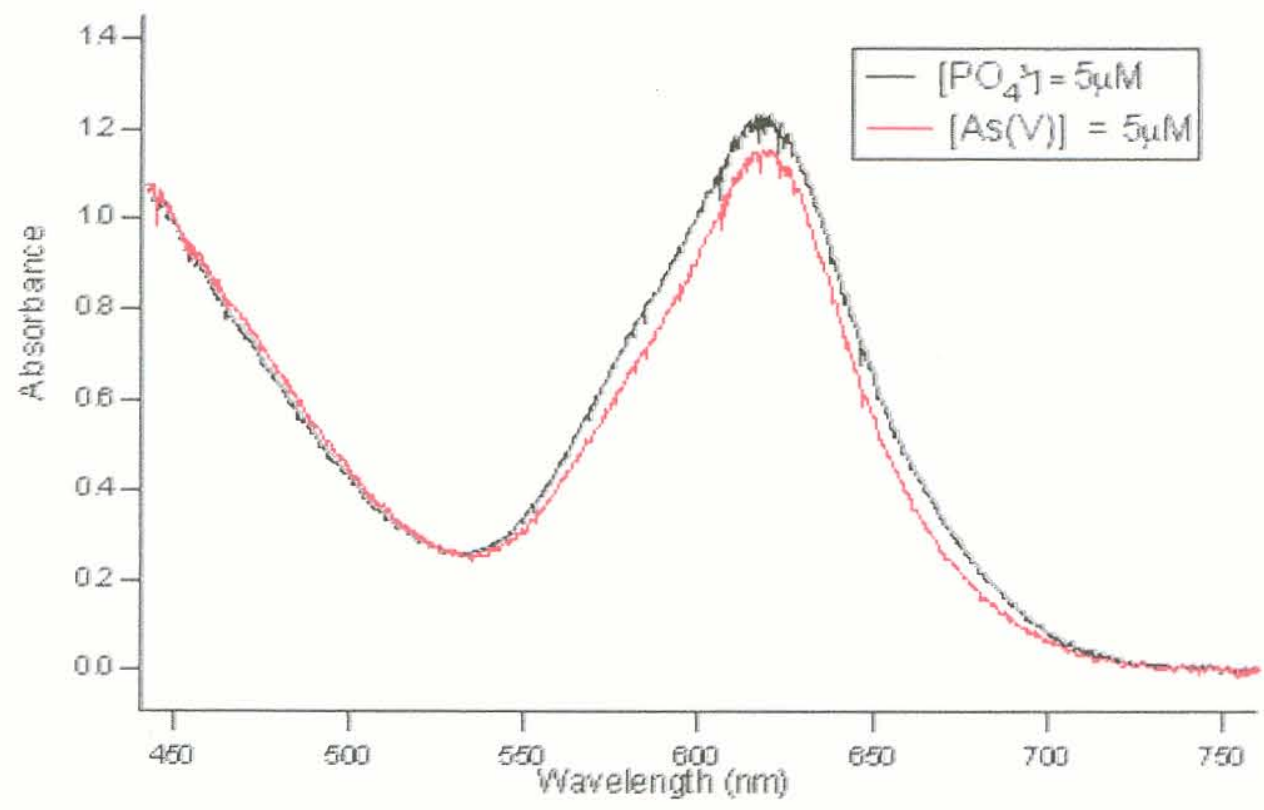

Figure 1-4 A comparison of the absorbtion spectra of phosphomolybdate and Arsenomolybdate complexes. The spectra from both complexes are indistinguishable at $630 \mathrm{~nm}$.

The presence of $\mathrm{As}(\mathrm{V})$ in a phosphate containing soil extract causes an increase in the absorbance at $630 \mathrm{~nm}$. Figure 1-5 shows the impact of $5 \mu \mathrm{M} A \mathrm{~s}(\mathrm{~V})$ on a solution of 5 $\mu \mathrm{M} \mathrm{O}_{4}{ }^{-3}$. The increase in the absorbance has a drastic affect on the final calculated concentration of phosphate because the soil extract is often diluted by a factor of 100 . For example if the sample shown below in Figure 1-5 is scaled for a dilution of 50, the final phosphate concentration of the spiked sample is $520 \mu \mathrm{M}$ as opposed to the accurate concentration of $235 \mu \mathrm{M}$. Therefore, the error is increased dramatically and may cause the misidentification of an anthropic soil. The phosphate and arsenate signals must be decoupled in order to accurately analyze the soil phosphate. 


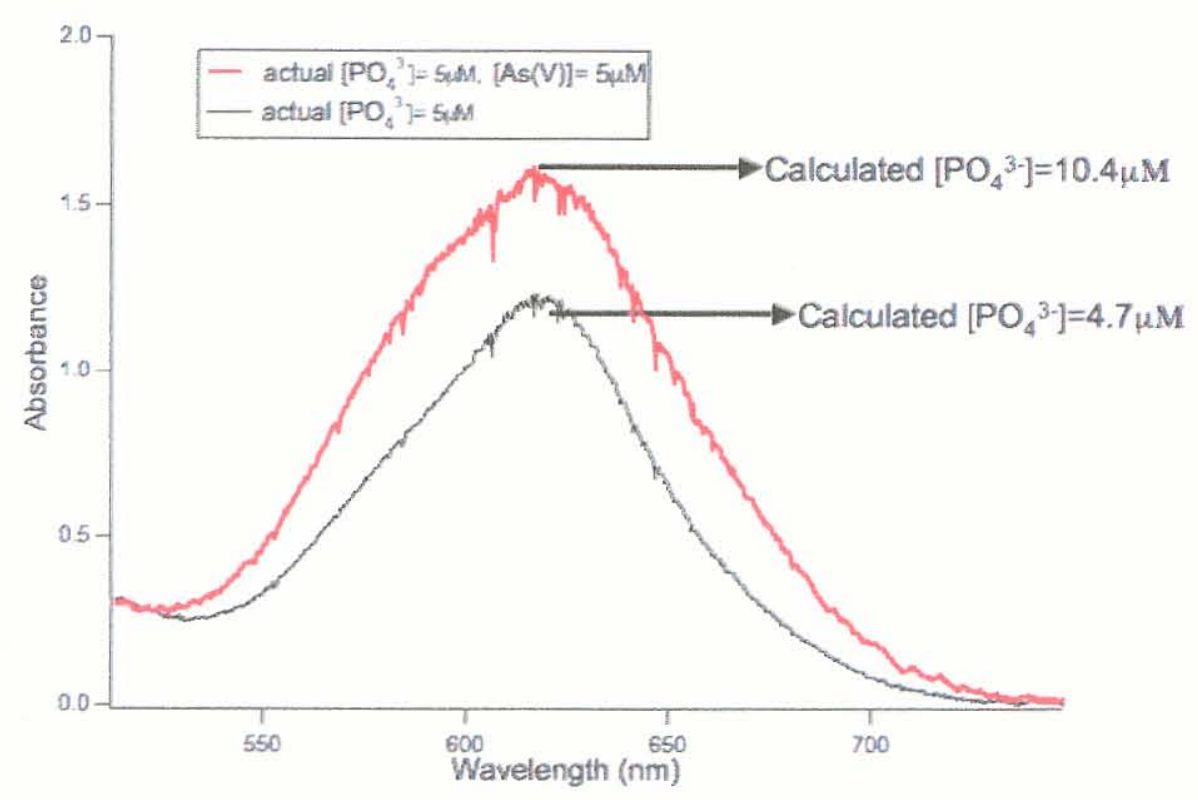

Figure 1-5 $\mathrm{As}(\mathrm{V})$ increases the absorbance and causes inaccuracies in phosphate concentration calculation. $\left[\mathrm{PO}_{4}{ }^{3-}\right]$ was calculated using the phosphate only calibration.

The kinetics of arsenate and molybdate complexation are complicated in the presence of phosphate. In solutions void of phosphate, As-Mo-MG forms slower than PMo-MG. However in the presence of phosphate, the rate of formation of the As-Mo-MG accelerates (Matsubara et al. 1987; Matsubara et al. 1990 ; Stauffer,1983). This makes it difficult to decouple the phosphate and arsenate signals temporally.

$\operatorname{As}(\mathrm{V})$ is the only chemical form of arsenic that interferes with the malachite green method. The reduced form of $\mathrm{As}(\mathrm{V})$, arsenite (As(III)), is incapable of binding malachite green and therefore does not interfere with phosphate analysis. A method for the reduction of the arsenate to arsenite is necessary for the accurate analysis of phosphate. 


\subsection{Arsenate and Soil}

Arsenate enters the environment from anthropogenic activities and from geochemical background. Arsenic, in all of its chemical forms, occurs in over 200 minerals naturally. Over time, rocks containing As minerals are weathered and As is deposited into the environment ( $\mathrm{Ng}$ et al. 2001, Pillai et al. 2000). Arsenic is also associated with sulphide ores and iron oxides found in soil (Lenoble et al. 2003). Baseline concentrations of As in soil range from $5-10 \mathrm{mg} \mathrm{kg}^{-1}$ (Frank, 2005; Matera, 2003; Smedley and Kinniburgh, 2002). These low levels of naturally occurring arsenate do not significantly affect phosphate analysis.

Additional arsenate is added to the soil through anthropogenic activities. Modernday activities such as mining, disposal of industrial waste, smelting of nonferrous metal ores, and other industrial processes increase the concentration of arsenate in the soil (Frank, 2005; Pillai et al. 2000; Shi et al. 2003). Areas in the proximity of mines report concentrations of As up to $27,000 \mathrm{mg} \mathrm{kg}^{-1}$ (Frank, 2005). The arsenate deposited by these activities is likely to be confined to the top soil. The interference can be avoided by excavating deeper layers of the soil horizon.

Historically, arsenate was used in pesticide, herbicides and fungicides (Shi et al. 2003 ). In the early 1900 s, lead arsenate was heavily used as a pesticide in apple orchards on the north eastern coast of the United States. Multiple studies have been conducted to determine the extent of arsenate contamination of New England soils. Some have claimed arsenic does not move beyond the top soil, while others have detected elevated levels of arsenic as deep as 47 inches (Newton, 2006). A recent study found most of the 
$\operatorname{As}(V)$ is contained within the first 8 inches of the soil. The average $A s(V)$ concentration throughout the first 8 inches of soil was $120 \mu \mathrm{g} \mathrm{kg}^{-1}$ soil (Newton, 2006).

In natural systems, arsenic occurs in four oxidation states: $(-3),(0),(+3)$ and $(+5)$. $\mathrm{As}(\mathrm{III})$ and $\mathrm{As}(\mathrm{V})$ are the most prevalent forms in soil (Matera, 2003). As(V) is the dominant oxide in soils with $\mathrm{pH}$ values between 4 and 7 . As(III) is found strongly sorbed to oxides in soils with $\mathrm{pH}$ values ranging from 7 to 10 (Manning et al. 2002; Matera, 2003; $\mathrm{Ng}$ et al. 2001). In areas prone to flooding, arsenic exists in organic forms such as dimethylarsenic acid (DMA) and monomethylarsenic acid (MMA).

Similar to phosphate, arsenate adsorbs to iron, aluminum and calcium oxides. Iron and aluminum oxides adsorb arsenate in acidic soils. Iron arsenate is the more common form found in the environment. In alkali soils, arsenate is sorbed to calcium oxides (Manning, 2002; Matera, 2003; Ng et al. 2001).

Arsenate is considered relatively immobile in soil. However, the extent of mobility is controlled by multiple factors. Changes in soil $\mathrm{pH}$, organic content of the soil and microbial activity alter the anion exchange capacity and thus the mobility of arsenate. For example, $\mathrm{As}(\mathrm{V})$ is strongly sorbed to iron or aluminum at low $\mathrm{pH}$ and if the $\mathrm{pH}$ of the soil increases, it becomes highly mobile. An increase in mobility results in $\operatorname{As}(\mathrm{V})$ moving deeper into the soil profile or moving to a new location.

As a result of $\operatorname{As}(\mathrm{V})$ sensitivity to $\mathrm{pH}$, it is easily extracted from the soil using mildly acetic solutions. Dilute perchloric, nitric, acetic and hydrochloric acids have successfully been used to extract arsenic species from soil. Ammonium nitrate has also been used in combination with hydrochloric acid to increase the amount of arsenic extracted (Cao, 2004; Jiang, 2005; Matera, 2003; Newton, 2006). As mentioned above in 
Section 1.3.1, perchloric acid and hydrochloric acid are common reagents used to extract phosphate from soil. Ammonium nitrate and nitric acid are both found in the Mehlich3 extracting reagent. Thus, it is possible for arsenate to be extracted from the soil as a result of the phosphate extraction method.

While all archaeological sites are not affected by As(V), it is clear that certain sites are contaminated with $\mathrm{As}(\mathrm{V})$ deposited by anthropogenic activities. In order to ensure an accurate analysis of soil phosphate, an $\mathrm{As}(\mathrm{V})$ reduction method must be employed. Multiple samples from the excavation site must be tested to determine if $A s(V)$ is present in the area. If the soils do not contain As(V), then the phosphate analysis can proceed without an $A s(V)$ reduction step. However, if the samples do contain $A s(V)$, every sample must be treated with an $\mathrm{As}(\mathrm{V})$ reducing agent before phosphate analysis. Thus, the reduction process must meet the requirements for onsite analysis methods and be compatible with the method of phosphate analysis.

\subsection{Arsenate Reduction Methods}

Since As(III) is toxic, environmental agencies are concerned with the speciation of arsenic in water and soil. Therefore, there are multiple methods for the reduction of arsenate to arsenite in soil and water solutions.

Several methods use potassium iodide as a reducing agent. If $\mathrm{KI}$ is used as the only reducing agent, reduction takes four to five hours to complete. The method also requires strong $\mathrm{HCl}$ solutions $(5-6 \mathrm{M} \mathrm{HCl})$ because the reaction is driven by the formation of $\mathrm{AsCl}_{3}$. The reduction is made more efficient by using $\mathrm{SnCl}_{2}$ used in combination with $\mathrm{KI}$. This reduction method requires significantly less time than $\mathrm{KI}$ 
alone but requires profuse shaking, strong acid solutions and an arsine generator. (Chen et al. 1992; Dasgupta et al. 2002). KI, with or without $\mathrm{SnCl}_{2}$, has never been used in combination with the malachite green method or other spectrophotometric methods.

Sodium thiosulfate and sodium thiosulfite have successfully been used as an $\mathrm{As}(\mathrm{V})$ reducing agent with spectrophotometric methods. For example, $\mathrm{Na}_{2} \mathrm{~S}_{2} \mathrm{O}_{3}$ (Figure 1-6) was used to reduce $\mathrm{As}(\mathrm{V})$ concentration under $225 \mu \mathrm{g} \mathrm{L^{-1 }}$ before water samples were analyzed with the ascorbic acid method.

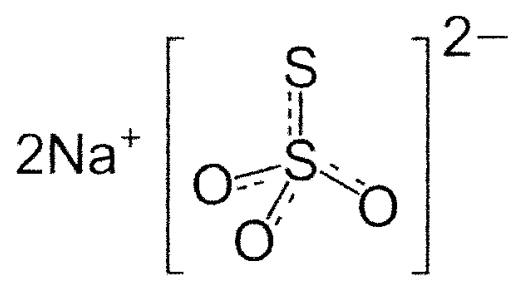

Figure 1-6: Structure of sodium thiosulfate.

$\mathrm{As}(\mathrm{V})$ reduction using sodium thiosulfate must occur in an acidic environment. A low $\mathrm{pH}$ is necessary for the formation of $\mathrm{S}_{2}$ gas, which reduces $\mathrm{As}(\mathrm{V})$ to $\mathrm{As}(\mathrm{III})$. Therefore, $\mathrm{Na}_{2} \mathrm{~S}_{2} \mathrm{O}_{3}$ is either prepared in a solution of $\mathrm{H}_{2} \mathrm{SO}_{4}$, or the $\mathrm{As}(\mathrm{V})$ sample is acidified before the $\mathrm{Na}_{2} \mathrm{~S}_{2} \mathrm{O}_{3}$ is added.

A wide range of reduction times have been reported for the thiosulfate and thiosulfite methods. Between eight and fifteen minutes are required for the reduction of As $(V)$ concentrations under $1 \mu \mathrm{M}$ using thiosulfate (Goulden and Brooksbank, 1974; 
Johnson, 1971). Carvalho et al. (1998) reported a reduction time of 3 hours was necessary to reduce concentrations of $\operatorname{As}(\mathrm{V})$ over $1 \mu \mathrm{M}$. In the case of thiosulfite, one hour is required for complete reduction of $\mathrm{As}(\mathrm{V}) 1.9 \mu \mathrm{M}$ (Linge, 2001).

There are several problems inherent in the thiosulfate reduction method. As the mechanism below shows, thiosulfate decomposes to form colloidal sulfur in an acid environment.

$$
\mathrm{S}_{2} \mathrm{O}_{3}{ }^{2-}(\mathrm{aq})+2 \mathrm{H}^{+}(\mathrm{aq}) \rightarrow \mathrm{S}(\mathrm{s})+\mathrm{SO}_{2}(\mathrm{~g})+\mathrm{H}_{2} \mathrm{O}(\mathrm{l})
$$

The formation of the colloid interferes with the development of malachite green. Malachite green binds to $S_{2}$, which decreases the amount of malachite green available to complex with phosphomolybdate. Linge et al. (2001) noted that because malachite green developing reagent is often in an acidic solution, colloidal sulfur formed immediately upon the addition of the developing reagent. To negate interference of colloidal sulfur, additional reagents, such as metabisulfate, can be added in excess (Johnson, 1971; Linge et al.2001).

Additionally, this reduction method is unsuitable for soils with high iron concentrations because Fe(III) binds with thiosulfate (Motomizu et al. 1988). The thiosulfate bound to $\mathrm{Fe}(\mathrm{III})$ is unavailable to reduce $\mathrm{As}(\mathrm{V})$. Therefore, complete reduction is not achieved in solutions containing high concentrations of iron.

A third option for $A s(V)$ reduction is the L-Cysteine method. This method has been used to pre-reduce $\mathrm{As}(\mathrm{V})$ before photometric detection of $\mathrm{As}(\mathrm{III})$ (Dasgupta et al. 2002; Shi et al. 2003). However, L-Cysteine (shown in Figure 1-7) is more commonly used in combination with analysis techniques coupled with hydride generation, such as 
hydride generation atomic absorption spectrometry (Chen et al. 1992; Shi et al. 2003; Shraim et al. 1999).

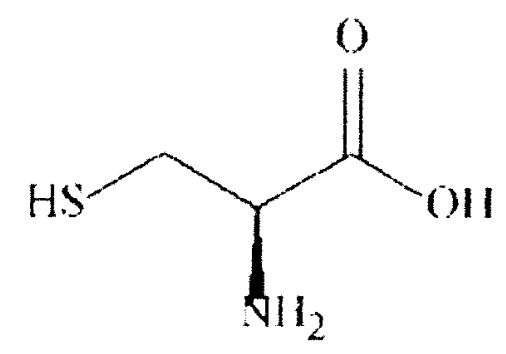

Figure 1-7: Structure of L-Cysteine

L-Cysteine reduces As(V) by forming thiolated As(III) complexes. L-Cystine and water are formed as byproducts.

$$
\mathrm{H}_{3} \mathrm{AsO}_{4}+5 \mathrm{HSR} \rightarrow \mathrm{As}(\mathrm{SR})_{3}+4 \mathrm{H}_{2} \mathrm{O}+\mathrm{RSSR}
$$

L-Cysteine is denoted as HSR and the oxidized form L-Cystine is denoted as RSSR.

Similar to thiosulfate, L-Cysteine reduction must occur in an acidic solution between a $\mathrm{pH}$ of 1 and 4 . In this $\mathrm{pH}$ range, L-Cysteine is protonated and capable of forming the thiolated As(III) complex. To obtain the proper $\mathrm{pH}$, the L-Cysteine solution is prepared in dilute $\mathrm{HCl}$ (less than $1 \mathrm{M}$ ). At room temperature, the reduction takes one hour to complete. However, heating the sample to $70-100{ }^{\circ} \mathrm{C}$ reduces the reduction time to as little as a minute (Carvalho et al. 1998; Chen et al. 1992; Shi et al. 2003; Tsalev et al. 2000). Raising the acidity of the solution can also shorten the reduction time. Chen et al. 
(1992) report that increasing the concentration of $\mathrm{HCl}$ from $0.02 \mathrm{M}$ to $1.0 \mathrm{M}$ decreased the reduction time of $35 \mathrm{nM} \mathrm{As}(\mathrm{V})$ from one hour to five minutes.

The $A s(V)$ reduction method must also meet the requirements for in-stride analysis and it must be compatible with the malachite green analysis method. The procedures described in the literature are all unsuitable for field work. $\mathrm{KI}$ and $\mathrm{Sn}_{2} \mathrm{Cl}$ methods are either too lengthy or require strong acids. Thiosulfate and L-Cysteine are the more promising methods for eliminating the interference of $\mathrm{As}(\mathrm{V})$ because both have been used with either malachite green or ascorbic acid analysis. However, thiosulfate is unsuitable because the metabisulfite required to prevent the formation of colloidal sulfur must be prepared daily. Thiosulfite does not form colloidal sulfur, but the required reduction time is not suitable for large scale surveys. L-Cysteine is only an efficient method at elevated temperatures, which is not feasible in remote locations.

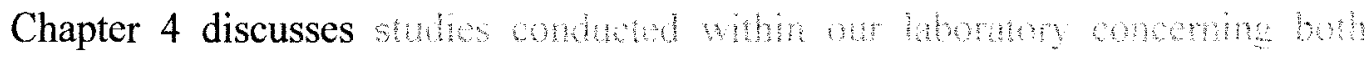

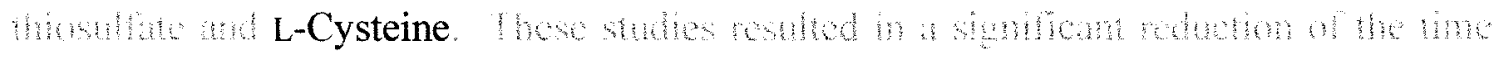
wationen of L-Cysteine reduction without increasing the solution temperature and a slight decrease in the thiosulfate reduction period

\subsection{Muti-elemental Analysis}

Phosphorus is only one of many elements deposited by human activities. For example, carbon, nitrogen, sodium and potassium are enriched in areas of human activity. However, these elements are highly susceptible to leaching, oxidation and plant uptake. Other elements, such as gold and mercury, are deposited during activities specific to a particular culture and are considered immobile and stable in the soil matrix. Analysis of 
these elements provides supplemental data that enhances the determination of intra-site usage and provides valuable information pertaining to cultural practices.

Undisturbed, abandoned soil floors are excellent candidates for multi-elemental analysis. Hutson et al. (2006) utilized multi-elemental analysis to study ancient domestic structures from a Mayan settlement in Mexico. Ancient Mayans commonly used a mercury sulfide known as cinnabar during burial rituals of the elite due to mercury sulfide's blood red color. Therefore, the determination of the areas with high $\mathrm{Hg}$ concentration was useful for locating Mayan burial sites. Huston et al. also found the concentration of $\mathrm{Hg}$ to be useful for locating burial sites. Two burials complete with offerings ( 70 jade and shell ornaments) were located in areas of high $\mathrm{Hg}$ concentration.

Cook et al. (2006) achieved similar success by analysis of the chemical components of two ancient Mayan soil floors in Guatemala. These studies focused on the analysis of trace heavy metals ( $\mathrm{Hg}$ and $\mathrm{Au}$ ) as well as rare earth elements. The $\mathrm{Au}$ concentration was determined to be unusually high and has become a point of contention in the archaeological society, as there is a consensus that gold was not used during Classic period Maya. Cook et al. were unable to draw definite conclusions about the origin of the $\mathrm{Au}$; however the $\mathrm{Au}$ enrichment is not the result of natural sources or modern human influence.

Rare earth elements (REE) are considered by many to be excellent indicators of past human activity. REE include Sc, Y, and the Lanthanide group. The exact anthropogenic source of REE enrichment is unknown, but it is suspected that the concentrations of REE are elevated by the decomposition of human teeth, hair, bone and skin (Cook et al. 2006; Enstwile et al. 2000). These elements are good indicators of 
human activity because the native concentration is low. However, enrichment of the REE is minimal (often between $1 \mathrm{ng} \mathrm{g}^{-1}$ soil and $1 \mu \mathrm{g} \mathrm{g}^{-1}$ soil) and can be difficult to detect.

The examples of multi-elemental analysis cited above were all conducted in a laboratory using either Inductively Coupled Plasma Mass Spectrometry (ICP-MS) or Inductively Coupled Plasma Atomic Emission Spectrometry (ICP-AES). The analysis process is confined to the lab due to the cumbersome instrumentation. However, the portable UV/Vis spectrometer can be exploited and used to detect many other elements and ions while onsite.

For example, $\mathrm{Fe}(\mathrm{III})$ can be detected through use of potassium thiocyanate (KSCN) (Aggrawal, 2003; Chriswell and Schilt, 1974; Ivsic and Tamhina, 2003; Kawakubo et al. 2004; Kopacek et al. 2001; Peters and French, 1941). The Fe[SCN $]_{3}$ complex is a vibrant orange and absorbs at a maximum of $450 \mathrm{~nm}$. The reaction is rapid and requires minimal amounts of chemicals and therefore it is an ideal method for fieldwork.

Iron is a major component of the earth's surface and is found in high concentrations in soil. Iron analysis alone is not conducive to locating former settlement sites. However if iron analysis is conducted in areas with high phosphate concentration or areas suspected of human habitation, valuable information about cultural practices is gained. For example, Terry et al. (2000) studied a Late Classic Mayan society in Aguateca, Guatemala by comparing the concentration of iron in specific sections of houses to the iron concentration at an off-site location. The analysis revealed several hot spots of iron, which indicates the area was used for the preparation of pigments and dyes. 
Terry et al. also discovered high concentrations of iron outside of the kitchen area. The accumulation of iron is believed to be the result of guards sharpening their machetes. It has been suggested that the butchering of animals leads to increased iron concentrations. However, the accumulation of iron from blood is not yet supported by chemical analysis (Cook et al. 2006).

Iron is just one example of the many elements that can be detected through UV/Vis analysis while onsite. Archaeologists and chemists must determine additional elements and ions that have archaeological relevance and can be detected efficiently using the portable spectrometer. Increasing the applications of the portable UV/Vis allows a more thorough analysis of the archaeological site in a timely and efficient manner.

\subsection{Conclusions}

Soil phosphate analysis has proven to be an important archaeological technique for delineating large archaeological sites and aiding the determination of intra-site usage. However, the quantitative analysis of phosphate is usually confined to a laboratory setting. In order to fully recognize the potential of phosphate analysis, the following objectives must be met:

1. Procedures for the extraction of phosphate from soil and the analysis of phosphate must be modified to increase the efficiency, portability and ease of use.

2. Arsenate is a chemical analogue of phosphate and interferes with phosphate detection methods. Due to both historic and modern anthropogenic activities elevated concentrations may be found in certain soils. Therefore an arsenate 
reduction method is necessary to ensure accurate phosphate analysis in contaminated soils.

3. Many elements, such as iron, can be detected through UV/Vis spectroscopy. Increasing the number of detectable elements allows for a more thorough analysis of the excavation site. Thus, it is important to develop in-stride methods for the detection of additional archaeologically relevant elements. 


\section{SOIL PREPARATION AND EXTRACTION}

\subsection{Introduction}

Malachite green phosphate analysis cannot be preformed on the soil directly without preliminary physical and chemical preparation. First, the water and organic debris is removed from the soil, and then phosphate is extracted from the soil using chemical reagents.

The recommended process for physical soil preparation is lengthy and not conducive for fieldwork. Ideally, soil is air dried in laboratories at room temperature. The time required for the drying process is dependent on the amount of moisture in the soil, ambient humidity, as well as the properties of soil. For example, clay soils high in organic content have lengthy drying periods when compared to sandy soils. Though heating the soil sample can expedite the drying process, heating the sample to temperatures above $40^{\circ} \mathrm{C}$ is inadvisable (Eliason, 1998). At elevated temperatures the chemical properties of the soil are altered and soil minerals - such as $\mathrm{K}, \mathrm{N}$, and organic $\mathrm{P}$ - become fixated or released. These changes lead to inaccurate results in the elemental analysis and thus oven drying is not commonly employed

The soil drying process must be altered in order for the Mehlich3 method to be integrated into a large-scale soil analysis. One possible solution is to forgo the drying process. This is potentially problematic as the introduction of water mass from the "wet" 
soil may introduce error to the calculation of phosphate and potentially yield low concentrations of calculated phosphate.

Several methods have been created for phosphorus extraction in order to specifically accommodate the diverse characteristics of soil. Accordingly, many fieldviable soil extraction reagents are limited to small $\mathrm{pH}$ ranges or unique soil propertiessuch as texture and phosphorus type. Situation specific extraction methods are generally incompatible with a broad range of archaeological sites containing diverse soil. Soils that have properties which deviate from the specifications of the selected extraction method will not be fully extracted and result in lower phosphate concentrations when analyzed. Therefore, in order to ensure accurate analysis, a versatile extraction method must be utilized.

\subsection{Extraction Methods}

The Mehlich3 method is an exception to the situation specific extraction. The extracting reagent is composed of $\mathrm{NH}_{4} \mathrm{~F}, \mathrm{NH}_{4} \mathrm{NO}_{3}$, glacial $\mathrm{CH}_{3} \mathrm{COOH}$ and $\mathrm{HNO}_{3}$. The combination of chemicals allows the extraction of available phosphorus from a variety of soil types and textures over a broad $\mathrm{pH}$ range without sacrificing the amount of extracted phosphorus. In fact, the Mehlich3 method extracts more available phosphorus than other methods with comparable methodology. For example, in acidic and neutral soils Mehlich3 phosphorus is 4\% more than Bray P1 phosphorus (Mehlich, 1984). Mehlich3 phosphorus in calcareous soils is notably higher than Olsen phosphorus. It has been reported to extract up to $12 \%$ more phosphorus than the Olsen method (Maiti and Das, 2007). As a result of the versatility and ease use of this extracting reagent, the Mehlich3 method has been widely adopted by soil scientists for use in the United States. 
Acid digestion methods are considered to be universal extracting reagents and can be used regardless of soil properties. Strong acids, such as $\mathrm{HClO}_{4}, \mathrm{H}_{2} \mathrm{SO}_{4}$ and $\mathrm{HCl}$, are used to remove phosphate from soil regardless of soil properties. These methods are widely used throughout the field of soil analysis because they are capable of extracting many soil chemical components and can be used for a variety of applications.

The percholoric acid method described by Olsen et al. (1982) uses $60 \% \mathrm{HClO}_{4}$ to extract both inorganic and organic phosphorus from soil. In order to achieve complete digestion of the soil, the acid and soil mixture is heated on a hot plate to a temperature just below the boiling point. This process must be performed in a hood to prevent exposure to toxic $\mathrm{HClO}_{4}$ fumes. The sample is heated until the insoluble material becomes white, which typically requires 40 minutes. The solution is diluted with distilled water once it has cooled. After the insoluble material settles to the bottom of the flask the solution can be analyzed to determine total soil phosphate.

Acid digestion methods that have been used in archaeological studies included the sulfuric acid and the hydrochloric acid methods. Hassan et al. (1981) used $7.5 \mathrm{~N}$ $\mathrm{H}_{2} \mathrm{SO}_{4}$ for the digestion, and the complete process requires 30 minutes. Cavanagh et al. (1988) used $\mathrm{HCl}$ to remove phosphorus from soil. The soil samples were heated in a steam bath for an hour digestion. The steam bath heating is not found in all $\mathrm{HCl}$ digestion procedures. Other published methods boil the soil and $\mathrm{HCl}$ mixture while others omit heating the sample during the digestion period.

Acid digestion methods are limited to laboratory analysis because of the hazardous nature of the concentrated acids. However the methodology of the Mehlich3 can easily be integrated into on-site phosphate analysis. This method does not require 
cumbersome equipment or extensive glassware. Also, it uses a small volume of a nonhazardous extracting reagent and the extracted soil is the only waste produced. Most importantly, the Mehlich3 method requires only five minutes for extraction. The limited time is well suited for archaeological surveys that analyze large numbers of soil samples. Despite the many advantages, the Mehlich3 method is limited by the soil preparation process.

\subsection{Reagents}

The Mehlich3 extracting reagent was prepared following the procedure outlined by A. Mehlich (1984) with one exception - EDTA was omitted from the stock solution. A stock solution of $3.75 \times 10^{-2} \mathrm{M} \mathrm{NH}_{4} \mathrm{~F}$ was prepareded in deionized water. The extraction solution was prepared by dissolving $20.0 \mathrm{~g}$ of $\mathrm{NH}_{4} \mathrm{NO}_{3}$ in approximately 800 $\mathrm{ml}$ of water. $40 \mathrm{ml}$ of stock solution, $11.5 \mathrm{~mL}$ of glacial acetic acid, and $0.840 \mathrm{ml}$ of concentrated nitric acid were added. The solution was mixed thoroughly and brought to a final volume of $1 \mathrm{~L}$.

The Olsen extracting agent was prepared following the procedure explained by Olsen and Sommers (1982). The solution contains $15 \mathrm{~mL}$ of $1 \mathrm{M} \mathrm{NH}_{4} \mathrm{~F}$ solution and 25 $\mathrm{mL}$ of $0.5 \mathrm{M} \mathrm{HCl}$. The extracting reagent is brought to a final volume of $500 \mathrm{~mL}$ using DI water.

Phosphate concentration of the extracted soil was determined using the malachite green method of phosphate analysis. Reagent 1 , a $3.0 \times 10^{-4} \mathrm{M}$ malachite green solution, was prepared from malachite green oxalate. $350 \mathrm{mg}$ of $89 \%$ Partially hydrolized polyvinyl alcohol were dissolved before the malachite green was added in order to prevent the precipitation of the dye. Reagent 2, 6.9 $\left.\times 10^{-3} \mathrm{M}\left(\mathrm{NH}_{4}\right)_{6} \mathrm{Mo}_{7} \mathrm{O}_{24} 4 \mathrm{H}_{2} \mathrm{O}\right)$ 
solution, was prepared in $3 \mathrm{M} \mathrm{H}_{2} \mathrm{SO}_{4}$. Further details on the malachite green method for phosphate determination are presented in Chapter 3 .

Extracting efficacy was determined using soil collected from the Blue Ridge Center for Environmental Stewardship (BRCES) in Loudon County, Virginia.

\subsection{Laboratory Preparation and Extraction}

In the laboratory, moisture was removed from the soil by heating the samples to temperatures between 40 and $70{ }^{\circ} \mathrm{C}$ for several hours in an oven. After the samples cooled and returned to room temperature, the soil was filtered using a metal screen to remove large organic debris and rock. The soil was crushed using a mortar and pestle and filtered with a metal screen. A sample prepared following this process can be stored indefinitely provided it remains in a dry environment (Jones, 2001). Therefore, Loudoun County soil samples that were not immediately extracted were stored in glass vials for later use.

Extracting solution was added to the dried soil samples using a 10:1 extractant volume to soil mass ratio as recommended by Jones (2001). The volume/mass ratio is a variation from the original Mehlich3 procedure, which used a 10:1 volumetric ratio. Both ratio methods are acceptable provided the ratio is used consistently throughout the samples. Following the addition of the extracting reagent, the sample was shaken for five minutes to ensure proper mixing and maximum extraction of phosphate. The samples are highly turbid after the 5 minute shaking period and must be filtered to prevent inaccuracies during phosphate analysis. The soil was removed from the extractant using syringe filtration. The syringe filtration system is shown in Figure 2-1. Syringe filtration 
was used over the traditional gravity filtration in order to decrease the time required for extraction. The pressurized filtration system allowed this step to be complete in under a minute for most soil samples, with typical filtration times around 10-15 seconds. This is a significant improvement over gravity filtration, which takes up to an hour depending on the soil properties. The filtrate was kept and used for malachite green phosphate analysis.

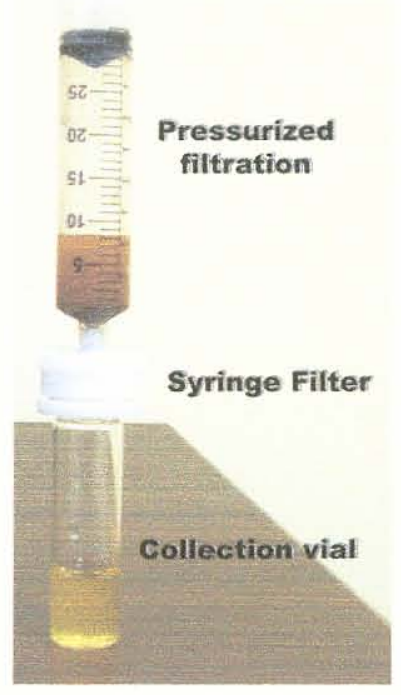

Figure 2-1: Syringe filtration of soil and extractant mixture. The solution in the collection vial is free of small soil particles and ready for phosphate analysis.

\subsubsection{Comparison of Extraction Methods}

A large quantity of soil was sampled from the BRCES land in a forested area free of human influence and was prepared following the procedure described above. The 
soil was stored in a desiccator to ensure the dried sample remained free of moisture From here forward, this sample is referred to as the "bulk sample". The phosphorus in the bulk sample was extracted using seven different methods used in archaeological surveys or soil analysis $\left(\mathrm{HClO}_{4}, \mathrm{H}_{2} \mathrm{SO}_{4}, \mathrm{HCl}\right.$ at several temperatures, Olsen and Mehlich3) to determine the accuracy of Mehlich3 extractions. The solution containing the extracted phosphate for each method was analyzed using the malachite green method The results of the phosphate analysis are shown in Table 2-1.

\begin{tabular}{|c|c|}
\hline Method & $\begin{array}{l}{\left[\mathrm{PO}_{4}\right]^{4-} \pm S . D^{\ni}} \\
(\mathrm{mg} \mathrm{P} / \mathrm{kg} \text { soil) }\end{array}$ \\
\hline $\mathrm{HClO}_{4}$ & $372 \pm 10$ \\
\hline $\mathrm{HCl} / S t e a m$ & $237 \pm 8$ \\
\hline $\mathrm{Hot} \mathrm{H}_{2} \mathrm{SO}_{4}$ & $226 \pm 5$ \\
\hline $\mathrm{Hot} \mathrm{HCl}$ & $146 \pm 3$ \\
\hline $\mathrm{HCl}$ & $93 \pm 1$ \\
\hline Mehlich 3 & $8 \pm 0$ \\
\hline Olsen & $7 \pm 0$ \\
\hline
\end{tabular}

Table 2-1. A Comparison of the phosphate concentration resulting from different extraction methods shows Mehlich 3 phosphate is significantly lower than the phosphate of acid digestions.

It is immediately obvious that the Mehlich3 extractable phosphate represents only a small fraction of the total phosphate extracted by the $\mathrm{HClO}_{4}$ method and is considerably lower than the $\mathrm{H}_{2} \mathrm{SO}_{4}$ and $\mathrm{HCl}$ extractable phosphate. Though the data seem to eliminate Mehlich 3 as an optimal extracting reagent, there are several important factors to consider. First, it is important to recognize that $\mathrm{HClO}_{4}$ method extracts both inorganic and organic phosphorus while Mehlich3 extracts only inorganic phosphorus. Thus, a portion 
of the difference in extracted phosphorus can be attributed to the organic content, which represents $50-80 \%$ of the total phosphorus in soil (Cornforth). This is particularly important because organic phosphorus data is irrelevant to archaeological work. It enters the soil from the decay of recent living organisms and does not have historical significance.

Estimation of the organic phosphorus content narrows the large gap between strong acid digestion phosphorus and Mehlich3 phosphorus, but the discrepancy still remains. It is clear that Mehlich3 does not extract the total inorganic phosphate. However, this archaeological survey is concerned with relative phosphate rather than absolute phosphate. A relative analysis compares the phosphate concentrations of multiple soil samples from the site to assign distinctions of high, medium and low phosphate. Then the relative amounts of phosphate are used to create a gradated spot map and used to distinguish intra-site boundaries and usage.

For a relative phosphate survey, Mehlich3 has several advantages over the other extraction methods. Recall the Mehlich3 soil digestion requires five minutes as opposed to the thirty-minute acid digestion analysis. This minimal time requirement allows for maximum efficiency when surveying expansive archaeological sites. Moreover, the Mehlich 3 method can be integrated seamlessly into fieldwork because it does not require additional instrumentation to heat the sample. Logistically, Mehlich3 is the more suitable choice for in-stride relative phosphate analysis

The Mehlich3 extract is more compatible with the malachite green method of analysis. The malachite green method is sensitive to changes in the $\mathrm{pH}$ of the solution. As Figure 2-2 shows, the intensity of the absorbance signal for molybdophosphate- 
malachite green is diminished at low $\mathrm{pH}$. Mehlich3 extracts have a slightly acidic $\mathrm{pH}$, but the $\mathrm{pH}$ is within the optimum range of 2-5.

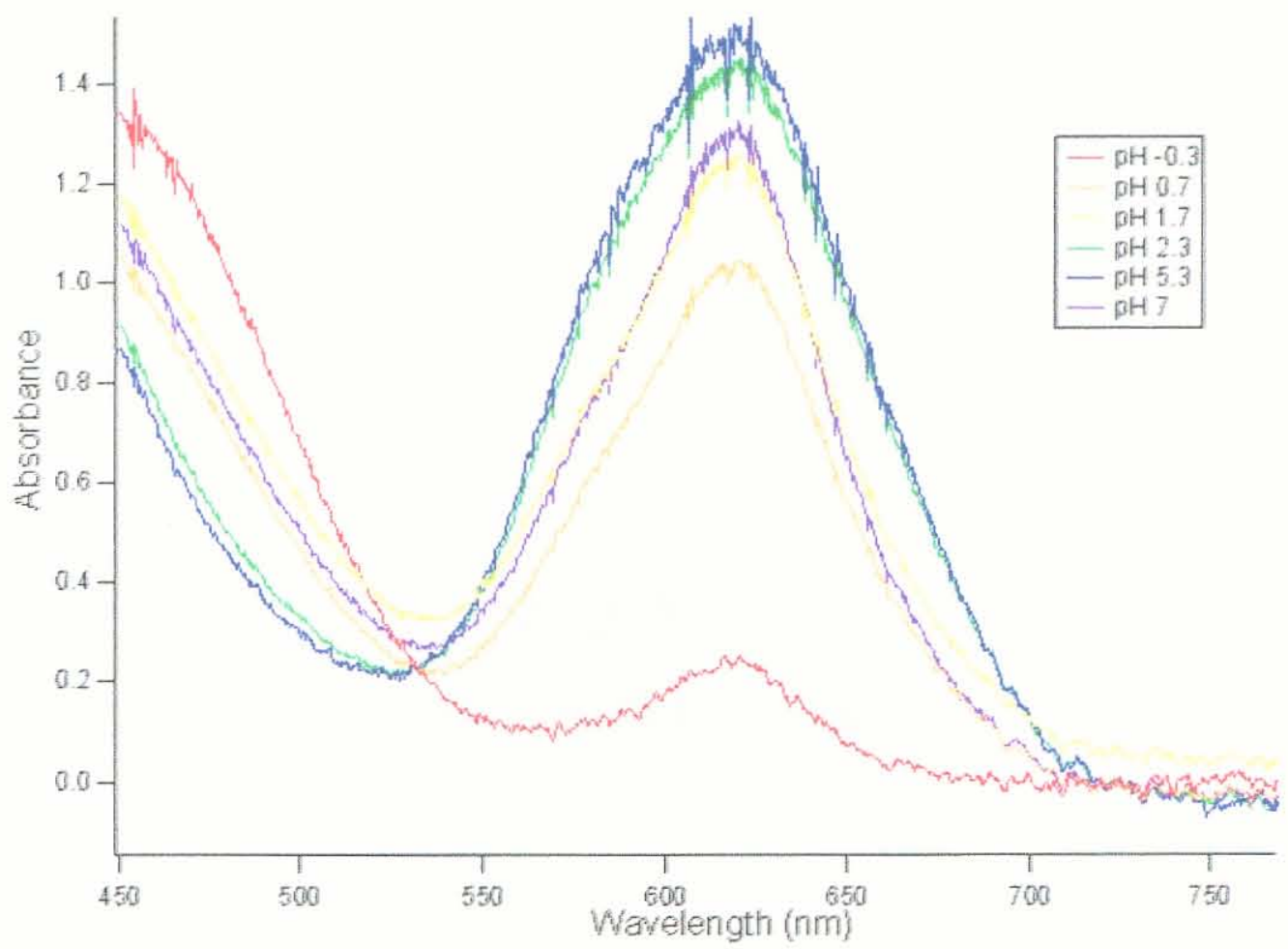

Figure 2-2: The absorbance of the malachite green and molybdophosphate complex is dependent on the sample $\mathrm{pH}$. Maximum intensity occurs in samples with $\mathrm{pH}$ between 2 and 5 .

Highly acid solutions with a pH below 2 show diminished absorbance at $630 \mathrm{~nm}$ and an increased absorbance at $460 \mathrm{~nm}$. The shift in absorbance reflects the change in the dye structure. At a low $\mathrm{pH}$ the malachite green becomes protonated, which decreases the conjugation of the electrons. Accordingly, this form of the dye absorbs at a shorter wavelength than the highly conjugated green form. This form cannot efficiently bind phosphomolybdate. The $\mathrm{HCl}, \mathrm{HClO}_{4}$, and $\mathrm{H}_{2} \mathrm{SO}_{4}$ extracts required extensive dilution to obtain a $\mathrm{pH}$ suitable for phosphate analysis. These large dilutions require additional time and introduce error into the calculation of phosphate. 
Unlike the acid digestion methods, in Mehlich3 extractions iron is not extracted from the soil (Jones, 2001). Although iron does not directly affect the malachite green method, it interferes with additional steps in soil analysis-such as the thiosulfate reduction of arsenate. A comparison of the extract solution color illustrates the variation in the amount of iron extracted (Figure 2-3). The more intense the yellow solution the more iron is present in the sample. Typically, the Mehlich3 phosphate extract is clear, whereas the phosphate extracts from the acid digestion methods are yellow indicating that iron (III) is present in the sample. The vibrant yellow extract solutions cannot be reduced with thiosulfate without an additional step to first remove the iron.

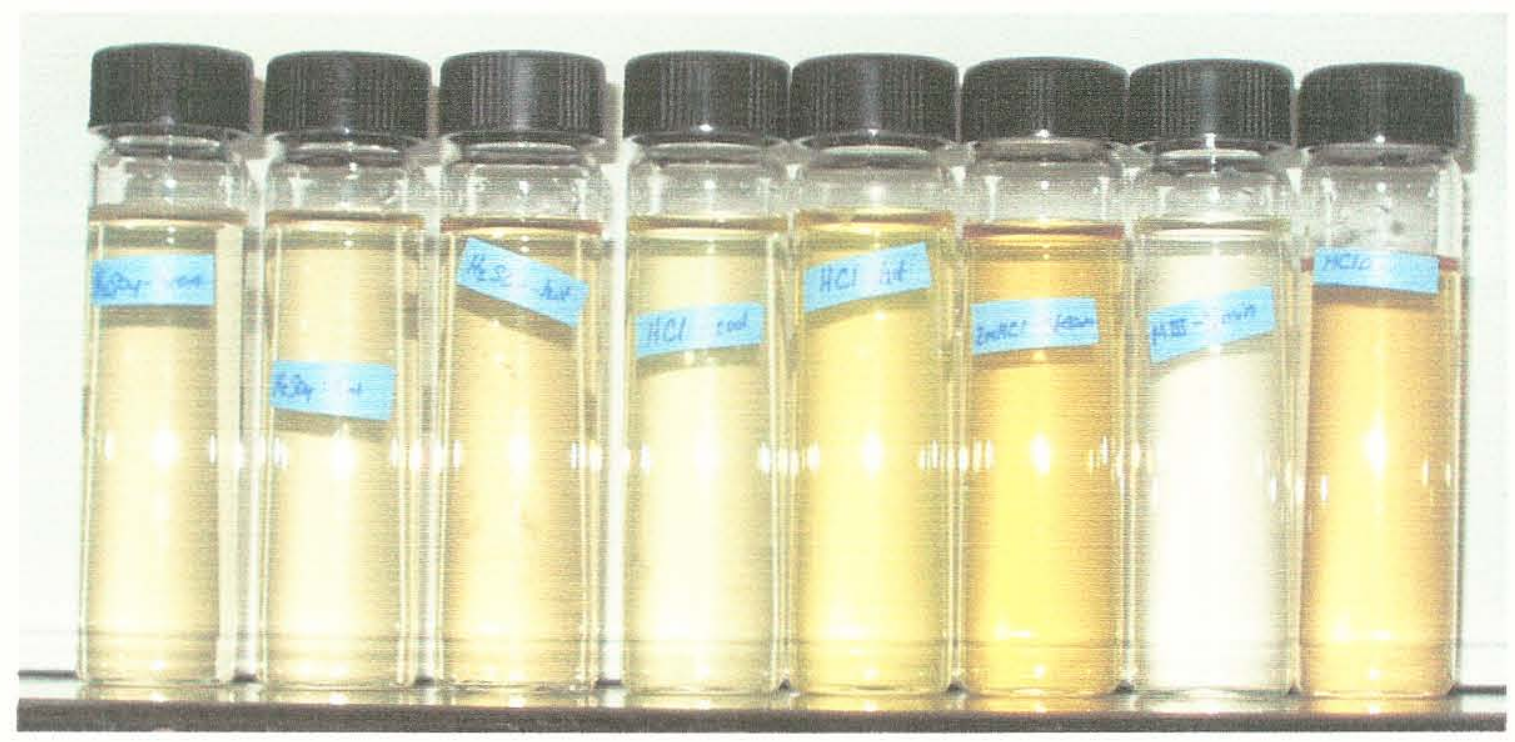

Figure 2-3. The filtrates from the different extraction methods show a wide variation in color. The Mehlich3 extract (second from right) is clear indicating iron is not present. $\mathrm{HClO}_{4}$ and $2 \mathrm{M} \mathrm{HCl}$ extracts have high concentrations of iron (indicated by intense yellow color). 


\subsubsection{Mehlich 3 Reproducibility}

Six soil samples were selected to represent various sample areas and soil composition. Three "on-site" samples (U028 and U026) and four "off-site" samples (471, 481, 493 and 511) from four different levels of sampling (A, B, L1, L5) were chosen. Each sample was extracted three times and then each extract was analyzed for phosphate concentration. The average phosphate concentration for each extract was compared to determine the reproducibility associated with the Mehlich3 method of extraction. Table 2-2 shows the resulting phosphate concentrations and standard deviation for three extractions of the soil sample.

\begin{tabular}{lr}
\hline Sample & $\begin{array}{r}{\left[\mathrm{PO}_{4}\right]_{\text {average }} \pm S . D^{\mathrm{a}}} \\
(\mu \mathrm{M})\end{array}$ \\
\hline 471B & $144 \pm 3$ \\
481B & $9 \pm 2$ \\
$493 \mathrm{~A}$ & $107 \pm 4$ \\
$511 \mathrm{~A}$ & $41 \pm 3$ \\
U028L1 & $388 \pm 27$ \\
U026L5 & $114 \pm 8$ \\
\hline $\mathrm{a}=3$ &
\end{tabular}

Table 2-2. Average phosphate concentration resulting from three Mehlich 3 laboratory extractions. The reproducibility for the extraction was acceptable for archaeological work.

The deviation from the average phosphate concentration is acceptable for archaeological purposes. The average relative standard deviation of the reported phosphate concentrations is $9 \%$. A portion of the error in reproducibility can be attributed to the large-scale dilutions necessary for phosphate analysis. Certain soil samples, such as U028L1, were diluted by a factor of 40 during phosphate analysis. Although the dilution was performed volumetrically, the dilution introduces error and 
decreased reproducibility. However, since the phosphate concentrations are used for a relative analysis, the deviations from the mean do not impact the identification of high phosphate concentration and anthropogenic soils. For example, despite the uncertainty in the phosphate concentration of U028L1 the sample can easily be identified as "high" concentration relative to the samples 493a and 511a.

The laboratory procedure for Mehlich3 extraction proved effective for soil samples from the archaeological site in Loudon County, Virginia. The oven drying process is ineffectual for field extraction and alternatives must be explored.

\subsection{In-stride Preparation and Extraction}

Soil extracted in the field was not dried during preparation in order to accommodate the time constraints of a dynamic, large-scale survey of the archaeological site. A significant amount of time was conserved by using the "wet" soil directly from the earth. In the field, a soil sample was taken such that it was free of rocks and organic debris, and then weighed without further preparation.

Extraction reproducibility for the wet soil was examined following the Mehlich3 extraction procedure described in section 2.3. The same six samples were extracted three times and the results are shown in Table 2-3.

\begin{tabular}{lr}
\hline Sample & $\begin{array}{r}{\left[\mathrm{PO}_{4}\right]_{\text {average }} \pm S . D^{\mathrm{a}}} \\
(\mu \mathrm{M})\end{array}$ \\
\hline $471 \mathrm{~B}$ & $131 \pm 4$ \\
$481 \mathrm{~B}$ & $2 \pm 1$ \\
$493 \mathrm{~A}$ & $63 \pm 0$ \\
$511 \mathrm{~A}$ & $17 \pm 1$ \\
U028L.1 & $370 \pm 20$ \\
U026L.5 & $94 \pm 2$ \\
\hline${ }^{\mathrm{a}} \mathrm{n}=3$ &
\end{tabular}

Table 2-3: Average phosphate concentrations determined from three Mehlich3 extractions of wet soils. 
The reproducibility of the phosphate concentration of an individual extraction was comparable to the dry extractions. The deviation of the phosphate for the three extractions was slightly higher $(13 \%)$ but remains acceptable for archaeological fieldwork. Therefore, it was determined that the addition of water mass did not greatly alter extraction reproducibility for the purposes of relative analysis. On a given day the amount of water in the soil is uniform throughout the sample. It is important to remain aware of weather conditions as changes in precipitation may alter the day to day degree of water content.

Undried soil samples contain a mass component that is exclusively water. Therefore, the total soil mass in $1.00 \mathrm{~g}$ of soil is actually somewhat lower depending on the water content, and it is expected that the concentration of phosphate in wet soils should be lower than phosphate concentration in dry soil. However, after comparing the phosphate concentrations derived from the wet and dry soils, it is clear there is a strong correlation between the field and laboratory methods (Figure 2-4.) 


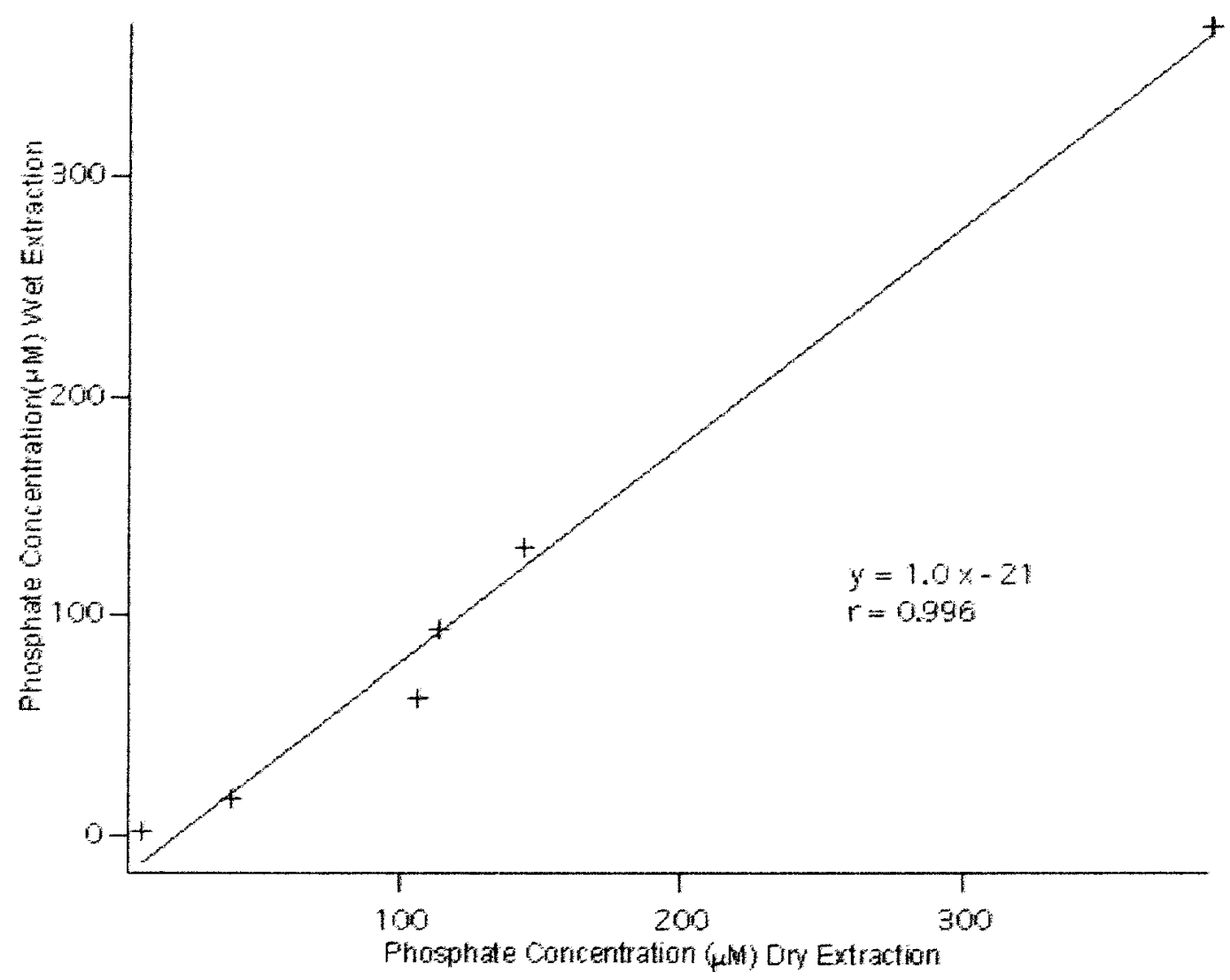

Figure 2-4. Phosphate concentrations calculated from wet and dry extractions show excellent correlation.

The accuracy loss resulting form the wet extraction does not affect the assignment of high phosphate. Sample U028L1 can still be assigned the classification of high phosphate relative to the wet extracted samples. However to ensure accurate analysis, the difference in extracted phosphate can be rectified by additional work in the field laboratory. The water content in a group of soil samples representing the survey area can be determined through oven drying. Then the percentage of water in the soil sample can be used to adjust the final calculation of phosphate concentration.

For example, consider an extraction of $1.00 \mathrm{~g}$ of wet soil yields a calculated phosphate concentration of $250 \mu \mathrm{M}$. Later in a field laboratory the soil is air-dried and it 
is determined that the soil contained $17 \%$ water by mass. The error resulting from the water mass can easily be corrected for in the calculation of $\mathrm{mg} \mathrm{P}^{-1}$ soil as shown below.

First, the actual mass of soil must be determined:

mass $_{d r y}=$ mass $_{\text {wet }}-\left(\right.$ mass $_{\text {wet }} \times \%$ water $)$

$=1 g-(\lg \times 0.17)$

mass $_{d r y}=0.83 g$

Using the dry mass of soil extracted, the accurate concentration in $\mathrm{mg} \mathrm{P} / \mathrm{kg}$ soil can be determined.

$250 \times 10^{-6} \mathrm{MPO}_{4}^{3-} \times(0.010 \mathrm{~L}) \times\left(\frac{1 \mathrm{molP}^{3}}{1 \mathrm{molPO}_{4}^{3-}}\right) \times\left(\frac{30,470 \mathrm{mg} P}{1 \mathrm{molP}}\right) \times\left(\frac{1}{8.3 \times 10^{-4} \mathrm{kgSoil}}\right)$

$=91.8^{m g P} / \mathrm{kgSoil}$

If the addition of water mass is not corrected, the concentration of $P$ in the soil is $76.1 \mathrm{mg} \mathrm{Pg} \mathrm{k}^{-1}$ Soil. For soils that have low water content, such as the example provided above, correcting for water mass is not a great concern. If weather conditions change during the survey, samples should be corrected for water mass in order to ensure accurate analysis. In the case of soil samples collected from Loudon County, the error introduced by water is minimal and the soil drying step was eliminated. 


\subsection{Conclusions}

Mehlich3 is a suitable reagent for the extraction of phosphate in archaeological soil samples. The amount of phosphorus cannot be considered an accurate assessment of the total soil phosphorus. Rather, the data must be used for a relative analysis to aid in the assignment of areas with high, medium and low phosphorus.

In order to increase the efficiency of a dynamic survey, the arduous soil drying process can be eliminated. Despite the added mass from water the wet soil extractions yield phosphate concentration results comparable to the laboratory preparation. The shortened preparation processes combined with a relative phosphate analysis are conducive to dynamic archaeological surveys. This type of analysis allows a greater number of soil extractions than traditional laboratory extraction methods. 


\section{3}

\section{PHOSPHATE DETECTION}

\subsection{Introduction}

Upon completion of the soil sample extraction process, the inorganic phosphate is typically analyzed using either colorimetric or spectrophotometric methods. The most sensitive and widely used detection methods for phosphate rely on the formation of the heteropolyacid phosphomolybdate in an acidic environment (Van Veldhoven and Mannaerts, 1986). After the macromolecule is formed, the phosphomolybdate is either reduced to the colored form or complexed with a cationic dye.

The reduction of phosphomolybdate results in the formation of molybdenum blue. The absorbance of the reduced phosphomolybdate can be correlated to the amount of phosphate in the solution. Originally, stannous chloride, tin and ascorbic acid were used as reducing agents (Broberg and Petterson, 1988; Murphy and Riley, 1962; Rao et al. 1997). Ascorbic acid was found to be the best reducing agent, although color development was slow. Murphy and Riley (1962) improved the ascorbic acid method by catalyzing the formation of molybdenum blue using antimony.

Alternatively, a cationic dye can be used to form an ion associated complex with

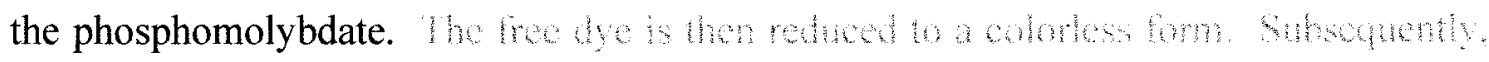

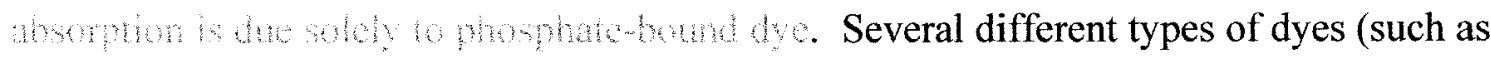
ethyl violet, malachite green, methyl green, and methylene blue) have successfully been 
used to detect phosphate (Broberg and Petterson, 1988; Motimizu et al. 1983; Murphy and Riley, 1962; Rao et al. 1997; Van Veldhoven et al. 1986;). Motomizu et al. (1983) determined malachite green was the most suitable dye for phosphate analysis because it did not form a precipitate as readily as the other dyes. Motomizu et al. also noted "the coloration [of malachite green] was the best of all dyes examined".

Both the ascorbic acid and the malachite green method are widely accepted by the soil science community, but the malachite green has several advantages. The malachite green method is at least four times more sensitive then the ascorbic acid method (Rao et al. 1997). The increase in sensitivity is attributed to the higher absorption coefficient of malachite green. Furthermore, the malachite green method is acknowledged as the more precise and accurate method, particularly when analyzing soils with low levels of phosphate (Broberg and Petterson, 1988; Rao et al. 1997). For these reasons, the malachite green method was selected for in-situ phosphate analysis of archaeological soil samples.

Malachite green has three different forms (Figure 3-1). The yellow, protonated form is found in solutions with $\mathrm{pH}$ below 2 , and the green form dominates at a $\mathrm{pH}$ above neutral. In slightly acidic environments both forms are found in nearly equal amounts. A colorless form results from the reduction of the dye.

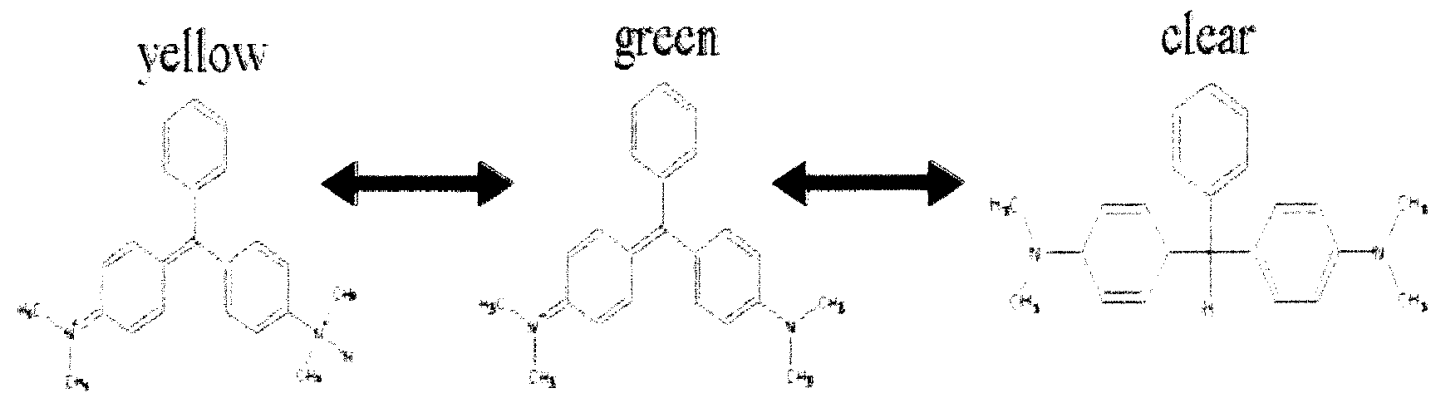

Figure 3-1: The structures of malachite green. 
Each form of malachite green has a unique absorbance spectrum (Figure 3-3). The reduced form is colorless and therefore shows no absorbance in the visible range. The natural green form of malachite green displays two peaks: a small peak at $425 \mathrm{~nm}$ and a more prominent peak absorbing at a maximum near $630 \mathrm{~nm}$. The yellow form absorbs strongly around $450 \mathrm{~nm}$.

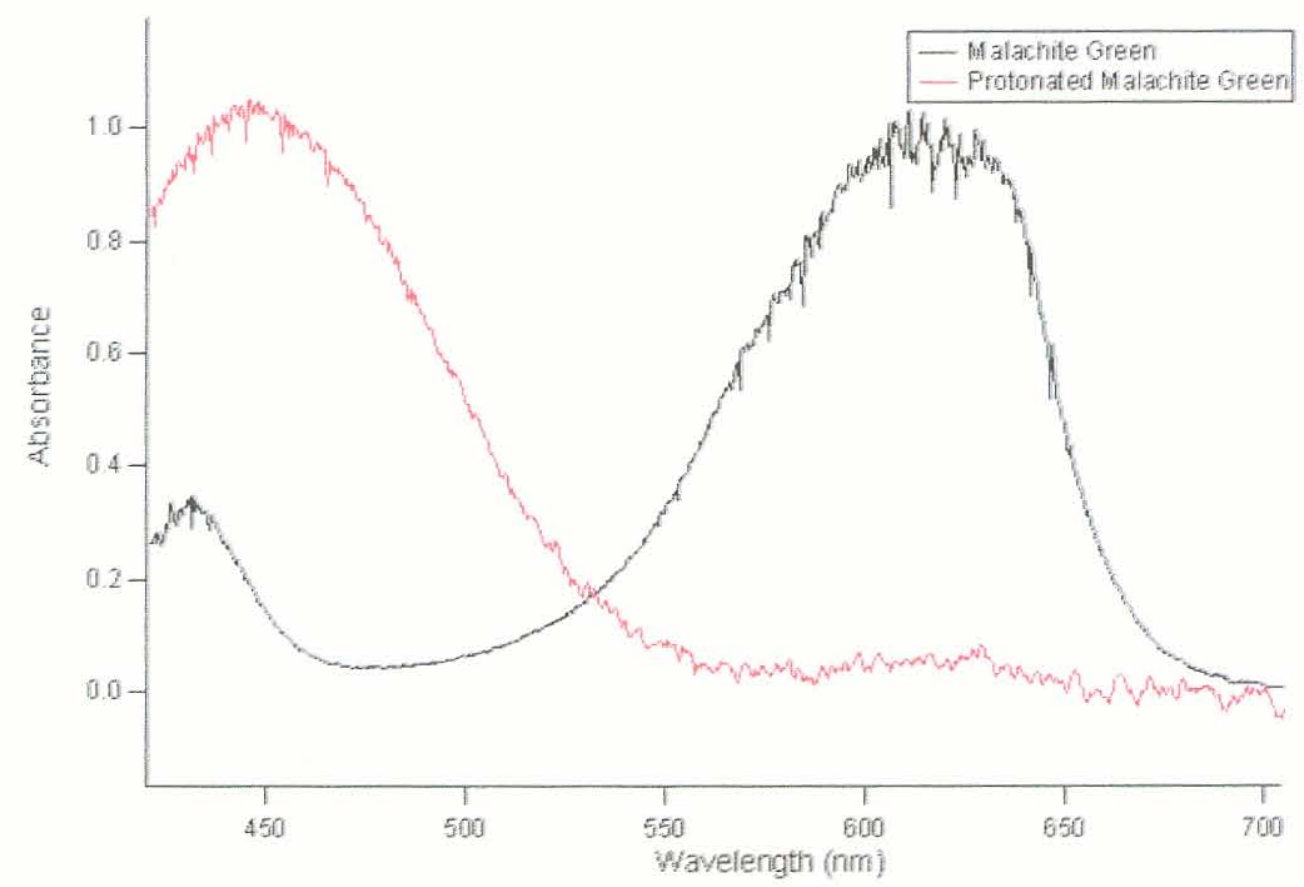

Figure 3-3: The absorbance spectra of the green and yellow forms of malachite green. Leuco-malachite green does not produce an absorbance spectrum.

Both the yellow and green forms are capable of forming a complex with phosphomolybdate. However, the yellow form displays a very mild propensity for bonding to the phosphomolybdate. The absorbance resulting from this complex is considered negligible compared to the absorbance from the green form. Since the phosphate concentration correlates with the green form, the absorbance is monitored in the $600-700 \mathrm{~nm}$ range. 
Sulfuric acid is used to reduce all unbound dye molecules to the colorless leuco form. This reduction process requires thirty minutes or longer to reach completion (Broberg and Petterson, 1988; Motomizu et al. 1983; Rao et al. 1997). The absorbance of the solution after this time period can be attributed solely to the phosphate-bound dye and the concentration can be determined.

Although the reagents in the malachite green method are more suitable for in-situ analysis than the ascorbic acid method, the thirty-minute development time is impractical for a large site where hundreds of soil samples are generated. The lengthy development time must be decreased before it can be incorporated into a large-scale survey. Kinetic studies in our laboratory have proven that the phosphate development time can be shortened significantly to three minutes. Further studies showed that by utilizing dual absorbance measurements, the analysis can occur instantaneously.

\subsection{Reagents and Instrumentation}

A stock solution of $3.0 \times 10^{-4} \mathrm{M}$ malachite green solution was prepared from malachite green oxalate. $350 \mathrm{mg}$ of $89 \%$ partially hydrolized PVA was dissolved in $80 \mathrm{~mL}$ of water. The addition of PVA prevents the malachite green from precipitating (Broberg and Petterson, 1988; Van Veldhoven et al. 1986). Next, $35 \mathrm{mg}$ of malachite green were added. Vigorous stirring ensured all malachite green was dissolved.

A $6.9 \times 10^{-3} \mathrm{M}$ Ammonium Heptamolybdate solution was prepared by dissolving $0.857 \mathrm{~g}$ of $\left(\mathrm{NH}_{4}\right)_{6} \mathrm{Mo}_{7} \mathrm{O}_{24} \cdot 4 \mathrm{H}_{2} \mathrm{O}$ in $60 \mathrm{~mL}$ of $3 \mathrm{M} \mathrm{H}_{2} \mathrm{SO}_{4}$ and brought to a final volume of $100 \mathrm{~mL}$ with water. 
Stock solutions of phosphate were prepared from $\mathrm{KH}_{2} \mathrm{PO}_{4}$ (Aldrich, 99\%), without further purification. Working solutions were prepared by volumetric dilution.

Absorbance measurements were obtained using an Ocean Optics fiber optic spectrometer for all absorbance measurements. A $10 \mathrm{~mm}$ pathlength cuvette was used Absorbance of the green form of malachite green was monitored $630 \mathrm{~nm}$. The absorbance of the protonated, yellow form was monitored at $473 \mathrm{~nm} .473 \mathrm{~nm}$ was chosen in order to avoid interference from the $450 \mathrm{~nm}$ peak of the green form.

\subsection{Three Minute Phosphate Analysis}

Established malachite green analysis methods utilize one developing solution containing all three of the necessary components (malachite green, molybdate, and sulfuric acid) (Rao et al. 1997; Van Veldhoven et al. 1986). This "premixed" solution has a low $\mathrm{pH}$ as a result of the addition of sulfuric acid, which implies malachite green will not exist in the natural green form. Instead after the solutions are mixed, there is a shift to the yellow, protonated form and the partial reduction of malachite green to the colorless form. The absorbance spectrum confirms the premixed developing reagent contains the yellow form, not the green form. (Figure 3-3). 


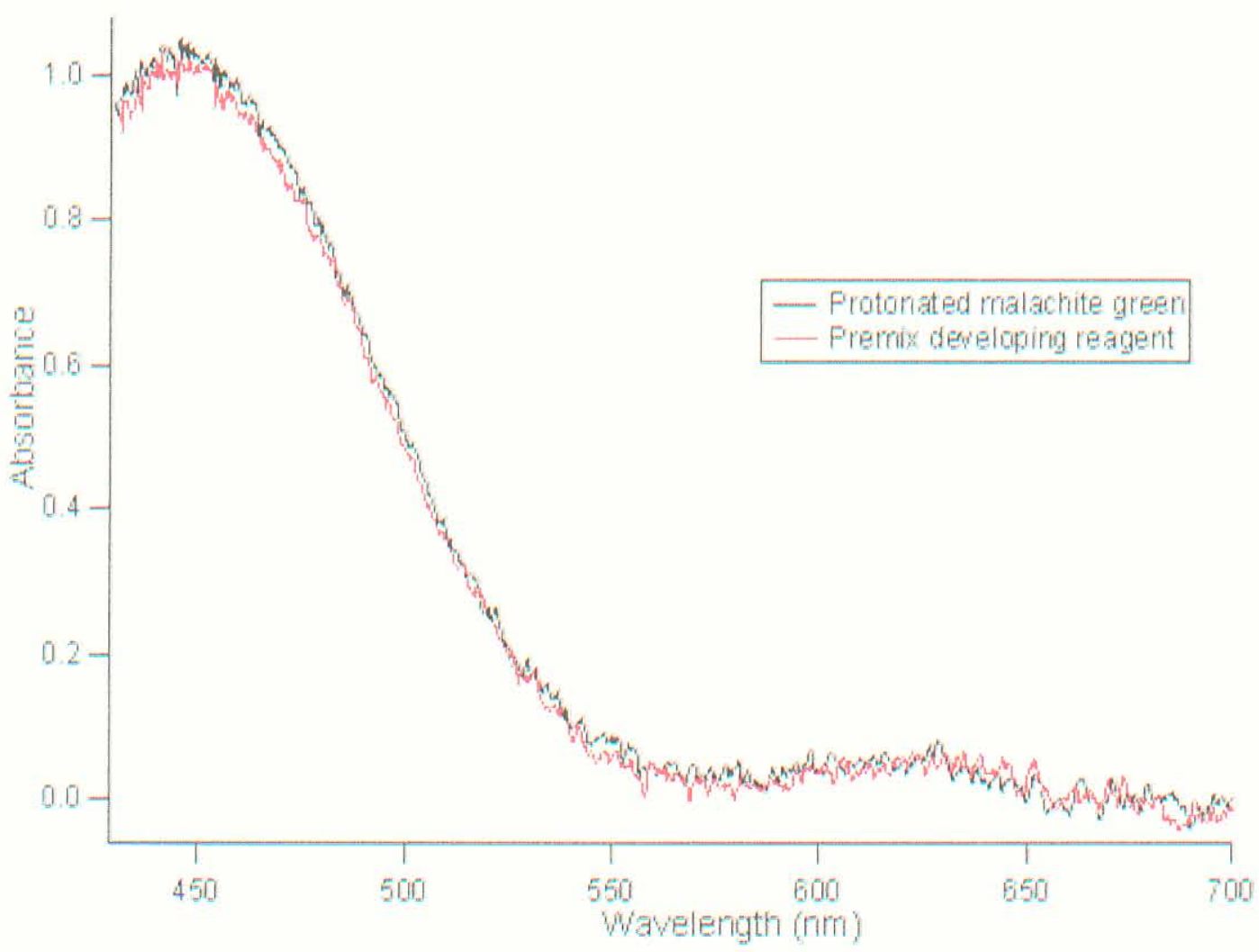

Figure 3-3: The absorbance spectrum of the premixed developing reagent shows that malachite green is present predominantly in its protonated form.

After approximately 10 minutes, the premixed solution reaches an equilibrium state between protonated malachite green (HMG) and the reduced leucomalachite green (LMG) by way of malachite green (MG).

$$
\mathrm{HMG} \leftrightarrows \mathrm{MG} \leftrightarrows \mathrm{LMG}
$$

When the mixture is added to a phosphate solution, phosphomolybdate is formed. The phosphomolybdate loosely associates with LMG and is then oxidized to the green form. The intensity of the green color is dependent on the rate of oxidation of the LMG and phosphomolybdate complex.

$$
-\frac{d \text { Green }}{d t}=k[\text { LMGPMo }]
$$


Only bound LMG molecules are oxidized back to the colored MG form and therefore, the rate of color formation is dependent on the phosphate concentration.

This complex process results in an asymptotic rise in absorbance over time as leucomalachite green binds to phosphomolybdate and oxidizes to the more stable colored form (Figure 3-4).

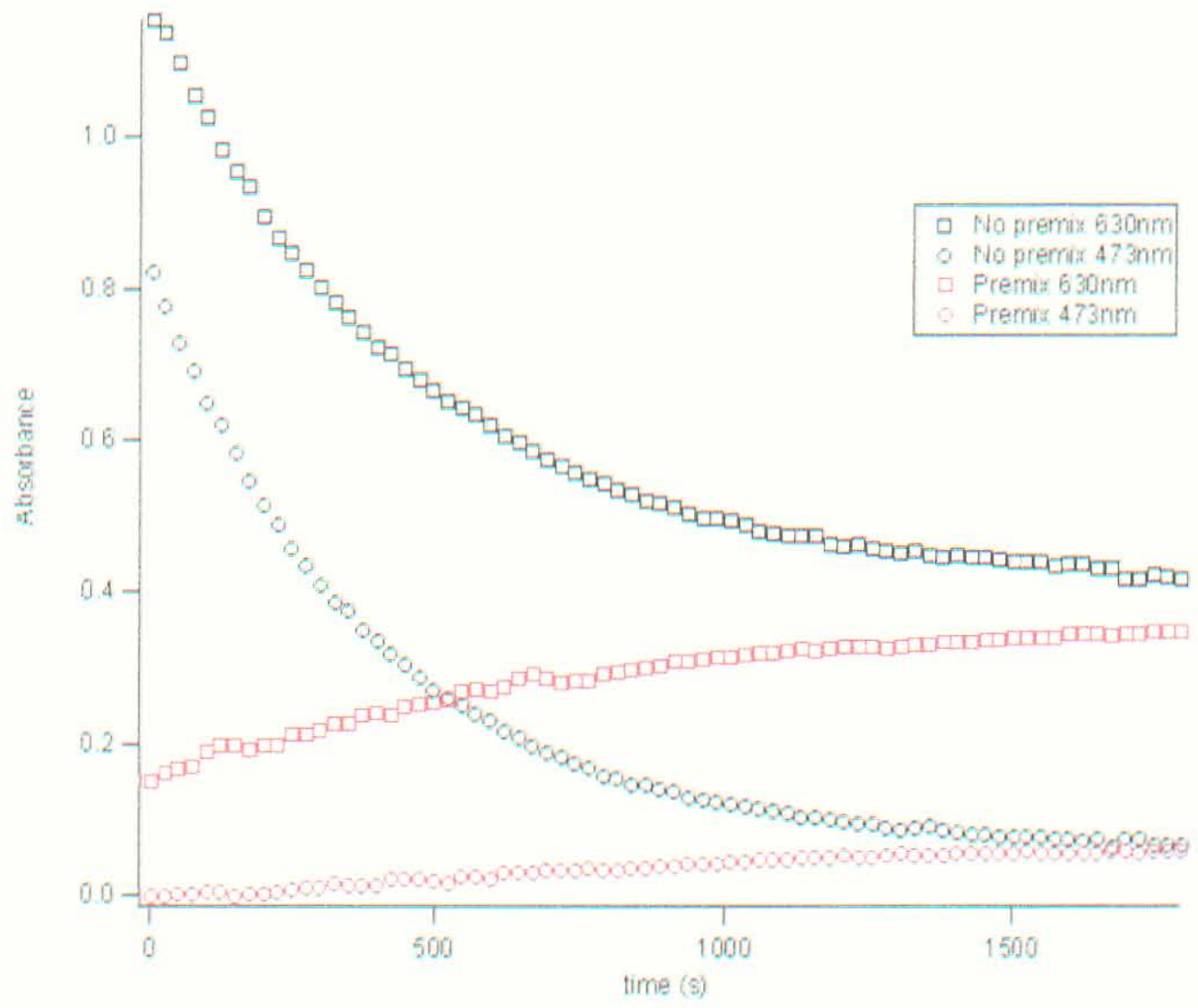

Figure 3-4: Kinetic analysis of the two methods of developing reagent addition. The premixed solution $(0, \square)$ displays an asymptotic increase in absorbance. The nonpremixed solutions $(0, \square)$ displays an exponential decay in absorbance.

The process can be simplified if the malachite green is added separately from the sulfuric acid. If the malachite green is kept separate from the sulfuric acid it exists as MG and HMG. After the malachite green is added to phosphate solution, loose association complexes are formed between the MG and phosphate ion (MGP). The 
formation of the MGP is essentially instantaneous. The total malachite green available $\left(\mathrm{MG}_{0}\right)$ is divided into $\mathrm{MG}$ and $\mathrm{MGP}$ :

$$
M G_{0}=M G+M G P
$$

The molybdate and reducing agent are added as a separate solution. The molybdate entraps the association complex while the sulfuric acid reduces the unbound MG. The rate of the color development is dependent on the reduction of the unbound malchite green.

$$
-\frac{d[M G]}{d t}=k[M G]
$$

Or

$$
-\frac{d[M G]}{d t}=k\left([M G]_{0}-[M G P]\right)
$$

MGP is equal to the concentration of phosphate, and MG can be considered $\left[\mathrm{PO}_{4}\right]$. Therefore,

$$
-\frac{d[M G]}{d t}=k\left([M G]_{0}-\left[P_{4}\right]\right)
$$

In the case of the separate addition of the solutions, the malachite green is in excess of the phosphate. The concentration of MGP is relatively small and can be neglected. The reduction of the unbound malachite green is effectively independent of the concentration of phosphate.

Separate addition of the developing reagents results in the exponential decay of the $630 \mathrm{~nm}$ absorbance over time (Figure 3-6). A relatively constant absorbance coefficient is achieved after approximately twenty minutes. However, a reproducible fraction of free malachite green is reduced after a given period of time. Therefore, an 
unknown sample of phosphate may be evaluated before the reaction reaches completion. In this manner, the development process can be shortened without loss in correlation or linearity of the phosphate analysis.

The validity of a shortened development time was examined by creating two calibrations using phosphate standards. $3 \mathrm{~mL}$ of phosphate solution were analyzed by adding $0.3 \mathrm{~mL}$ of the malachite green reagent and $0.3 \mathrm{~mL}$ of the sulfuric acid and molybdate solution. The absorbance was measured after 3 minutes and again after one hour. The 3 minute development time was selected after observing it as a point where the absorbance decay became less rapid, thereby providing an early time at which small timing errors would introduce a lesser degree of accuracy problems. The 1 hour time period was selected for comparison and was sufficient to ensure the reduction process of malachite green was complete.

The calibrations are shown in Figure 3-5. It is immediately obvious that the absorbance at 3 minutes is higher than the absorbance at one hour. This can be attributed to the incomplete reduction of unbound malachite green. However, the increased absorbance did not affect the linearity and correlation of the 3-minute calibration. The 3minute calibration line has a regression value of 0.996 and a slope of 0.071 , which is comparable to 1 -hour method (0.996 and 0.079 respectively). The parallel slopes indicate the shortened time period does not lead to a loss of sensitivity in the detection. The 3 minute readings are slightly less precise than those taken after an hour. The average relative standard deviation of 3-minute readings is 0.07 as opposed to 0.01 for 1hour measurements. The shortened method is sensitive to alterations in the data 
acquisition time because the reduction process is incomplete. Thus, a delay of several seconds causes a lower absorbance measurement and in turn a decrease in precision.

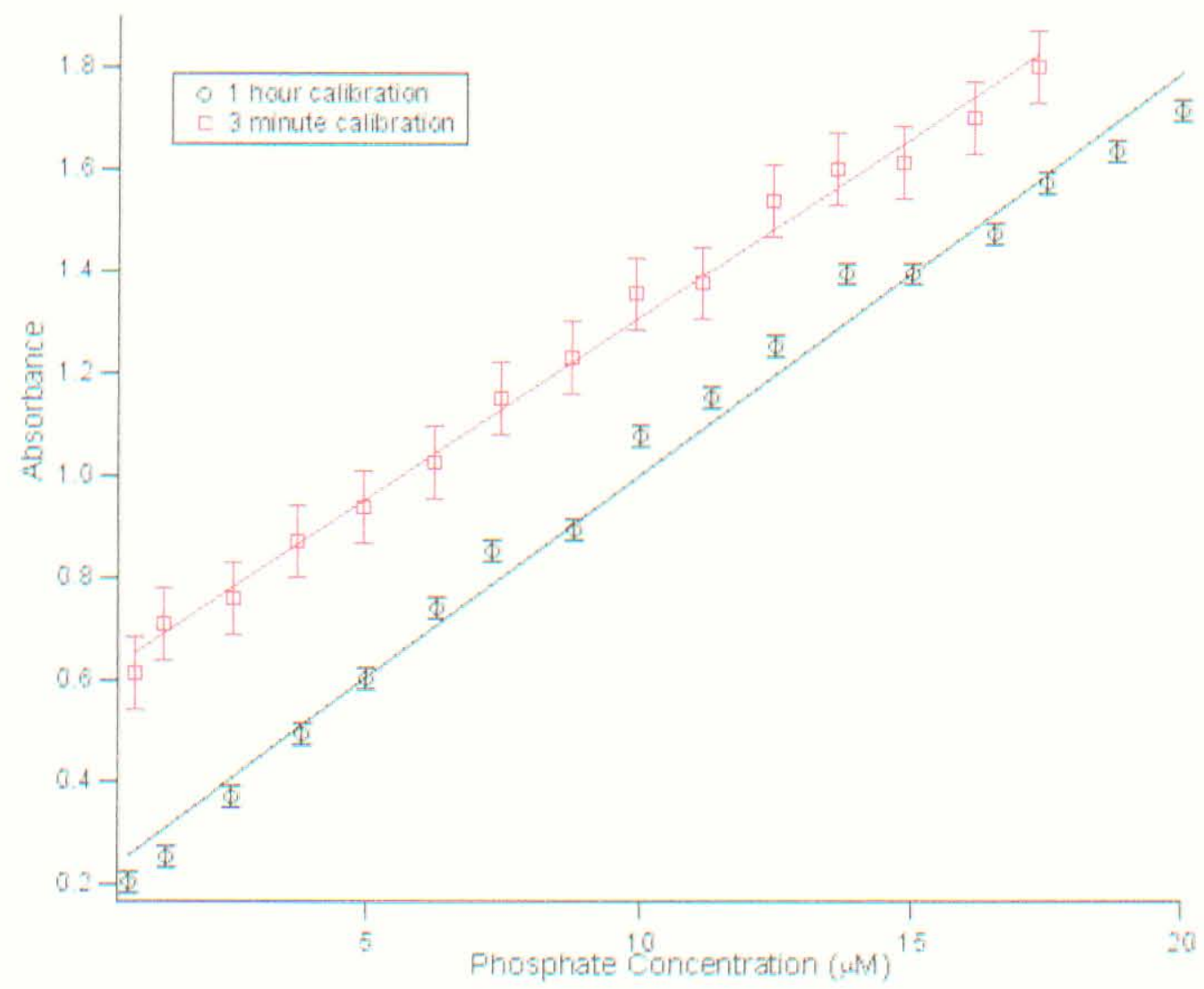

Figure 3-5: A comparison of 3 minute and 1 hour development shows the shortened analysis time does not lead to a significant loss in precision, correlation or sensitivity.

Despite the small loss in precision, the 3 minute development time is ideal for an archaeological survey. It is important to remember the archaeological analysis is concerned with relative phosphate and the small increase in deviation does not affect the assignment of "high" and "low" phosphate. The error can be corrected by analyzing additional samples at a later time. 


\subsection{Instantaneous Dual Absorbance Analysis}

The 3-minute procedure was employed for the field studies in Loudoun Co, and for all initial studies. Further kinetic studies of the development process led to an additional shortening of the acquisition time.

When the malachite green reagents are added to a phosphate solution separately, the absorbance profile is composed of two peaks resulting from both malachite green and protonated malachite green (Figure 3-8). The two peaks have maximum absorbance coefficients at $450 \mathrm{~nm}$ and $630 \mathrm{~nm}$.

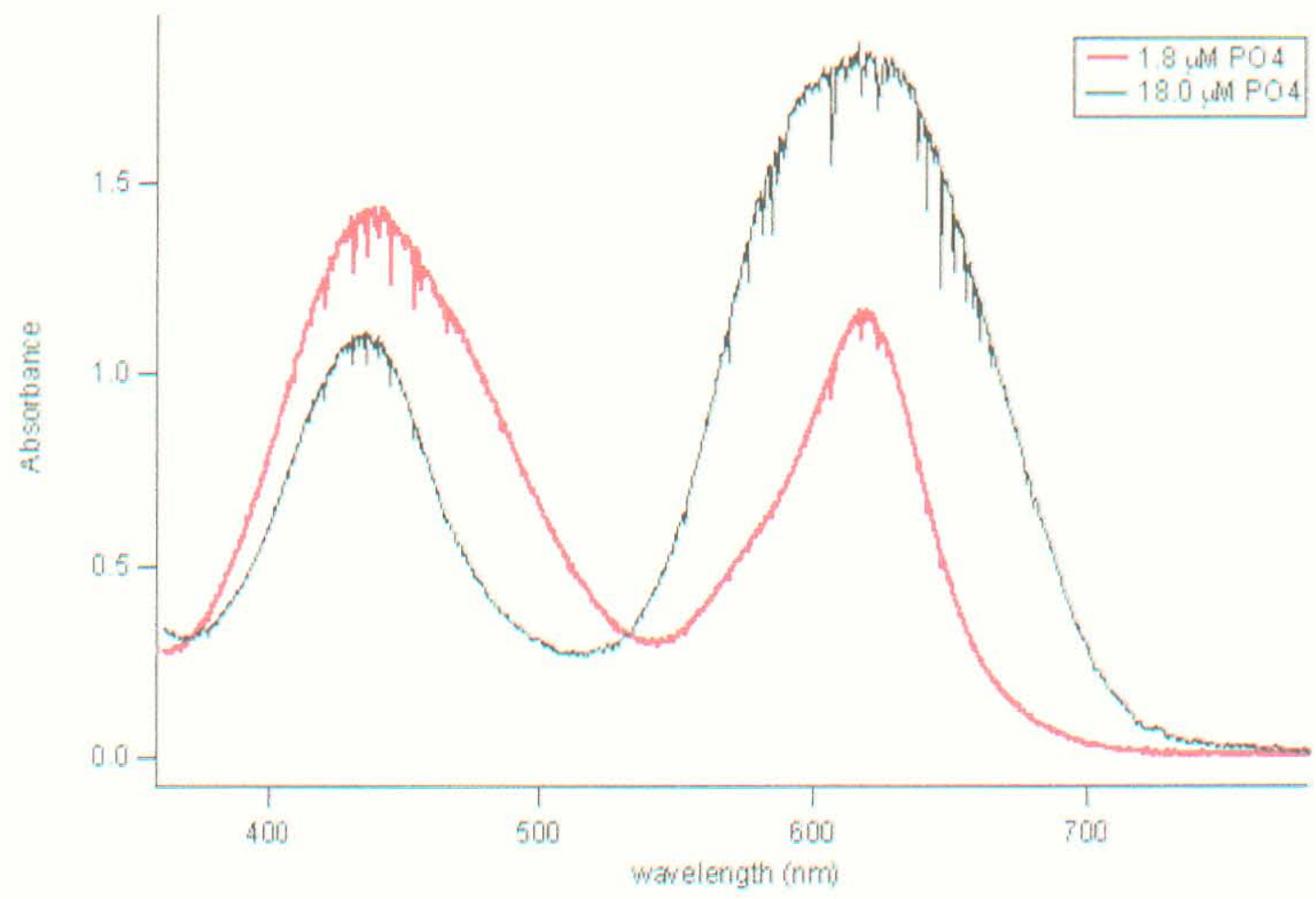

Figure 3-6: The spectra of two different phosphate concentrations show absorbance at $450 \mathrm{~nm}$ and $630 \mathrm{~nm}$. Absorbance at both peaks is dependent on phosphate concentration.

It is clear from Figure 3-6 that the absorbance of both peaks is dependent on phosphate concentration. At high phosphate concentrations, the peak at $630 \mathrm{~nm}$ is more 
intense than the peak at $450 \mathrm{~nm}$. At lower concentrations, the reverse is true. The shift from the yellow form to the green is controlled by the concentration of phosphate since bound malachite green is removed from the yellow/green equilibrium equation. When malachite green binds phosphomolybdate the yellow/green equilibrium shifts to produce the green form. As the phosphate concentration increases the yellow/green equilibrium shifts to produce more green form and the intensity of the $630 \mathrm{~nm}$ peak increases. Since the intensity of both peaks is controlled by phosphate, the difference between the absorbance coefficients can be used for phosphate determination. Recall from Figure 3-3 that malachite green has a small peak in the $400 \mathrm{~nm}-450 \mathrm{~nm}$ range, thus accurate absorbance measurements cannot be obtained. Instead, the absorbance of the protonated form is measured at $473 \mathrm{~nm}$ where the malachite green exhibits a window of low absorbance.

To understand how dual absorbance can be used to determine the phosphate concentration, it is important to understand each absorbance individually. Malachite green analysis conforms to Beer's law and therefore the absorbance from the protonated malachite green is:

$$
A_{473}=\varepsilon_{y}([H M G])
$$

The rate of formation of HMG can be written as:

$$
\frac{[M G]\left[H^{+}\right]}{M G H}=K_{a}
$$

Which can be rearranged:

$$
H M G=\frac{[M G]\left[H^{+}\right]}{K_{a}}
$$

Or, 


$$
H M G=[M G] K^{\prime}
$$

where $K^{\prime}=\frac{\left[H^{+}\right]}{K_{a}}$.

Equation 3-9 can be combined with Equation 3-8 to give:

$$
A_{473}=\varepsilon_{y} K^{\prime}[M G]
$$

Recall that the absorbance at $630 \mathrm{~nm}$ is written as:

$$
-\frac{d[M G]}{d t}=k\left(\left[M G_{0}\right]-[M G P]\right)
$$

The difference between the two absorption coefficients can be used to determine the phosphate concentration.

$$
A b s_{630}-A b s_{473}=\left(\varepsilon_{g}([M G]-[M G P])-\left(\varepsilon_{y} K^{\prime}[M G]\right)\right)
$$

Equation 12 can be rearranged:

$$
A_{630}-A_{473}=[M G]\left(\varepsilon_{g}-\varepsilon_{y} K^{\prime}\right)+\varepsilon_{g}[M G P]
$$

Recall that:

$$
[M G]=\left[M G_{0}\right]-[H M G]-[M G P]
$$

$[H M G]=[M G] K^{\prime}$ and therefore:

$$
[M G]=\left[M G_{0}\right]-[M G] K^{\prime}-[M G P]
$$

Equation 3-15 can be rearranged:

$$
[M G]\left(1+K^{\prime}\right)=\left[M G_{0}\right]-[M G P]
$$

Thus, the $[M G]$ can be written:

$$
[M G]=\frac{\left[M G_{0}\right]-[M G P]}{\left(1+K^{\prime}\right)}
$$

Equation 3-17 can be substituted into equation 3-13 to give: 


$$
A_{630}-A_{473}=\frac{\left[M G_{0}\right]-[M G P]}{1+K^{\prime}}\left(\varepsilon_{g}-\varepsilon_{y} K^{\prime}\right)+\varepsilon_{g}[M G P]
$$

Equation 3-18 is rearranged to:

$$
A_{630}-A_{473}=\varepsilon_{g}[M G P]-\frac{\varepsilon_{g}[M G P]}{1+K^{\prime}}+\frac{K^{\prime} \varepsilon_{y}[M G P]}{1+K^{\prime}}+\frac{\left[M G_{0}\right]}{1+K^{\prime}}\left(\varepsilon_{g}-\varepsilon_{y} K^{\prime}\right)
$$

The $[\mathrm{MGP}]$ can be isolated from the equation:

$$
A_{630}-A_{473}=[M G P]\left(\varepsilon_{g}-\frac{\varepsilon_{g}}{1+K^{\prime}}+\frac{K^{\prime} \varepsilon_{y}}{1+K^{\prime}}\right)+\frac{\left[M G_{0}\right]}{1+K^{\prime}}\left(\varepsilon_{g}-\varepsilon_{y} K^{\prime}\right)
$$

Equation 3-20 is in the form of $y=m x+b$ where $[M G P]=x, A_{630}-A_{473}=y$, $\frac{\left[M G_{0}\right]}{1+K^{\prime}}\left(\varepsilon_{g}-\varepsilon_{y} K^{\prime}\right)=\mathrm{b}$ and $\left(\varepsilon_{g}-\frac{\varepsilon_{g}}{1+K^{\prime}}+\frac{K^{\prime} \varepsilon_{y}}{1+K^{\prime}}\right)=\mathrm{m} . \quad$ A plot of the difference between $A_{630}$ and $A_{473}$ and the concentration of phosphate is linear. The relationship between the absorbance difference and phosphate concentration can be calibrated and used to determine the concentration of unknown phosphate solutions.

Kinetic analysis shows that for a given concentration, the absorbance difference between the two peaks is relatively constant (Figure 3-7). Therefore, the analysis time can be shortened to an instantaneous data acquisition provided the difference between the peaks correlates with phosphate concentration. 


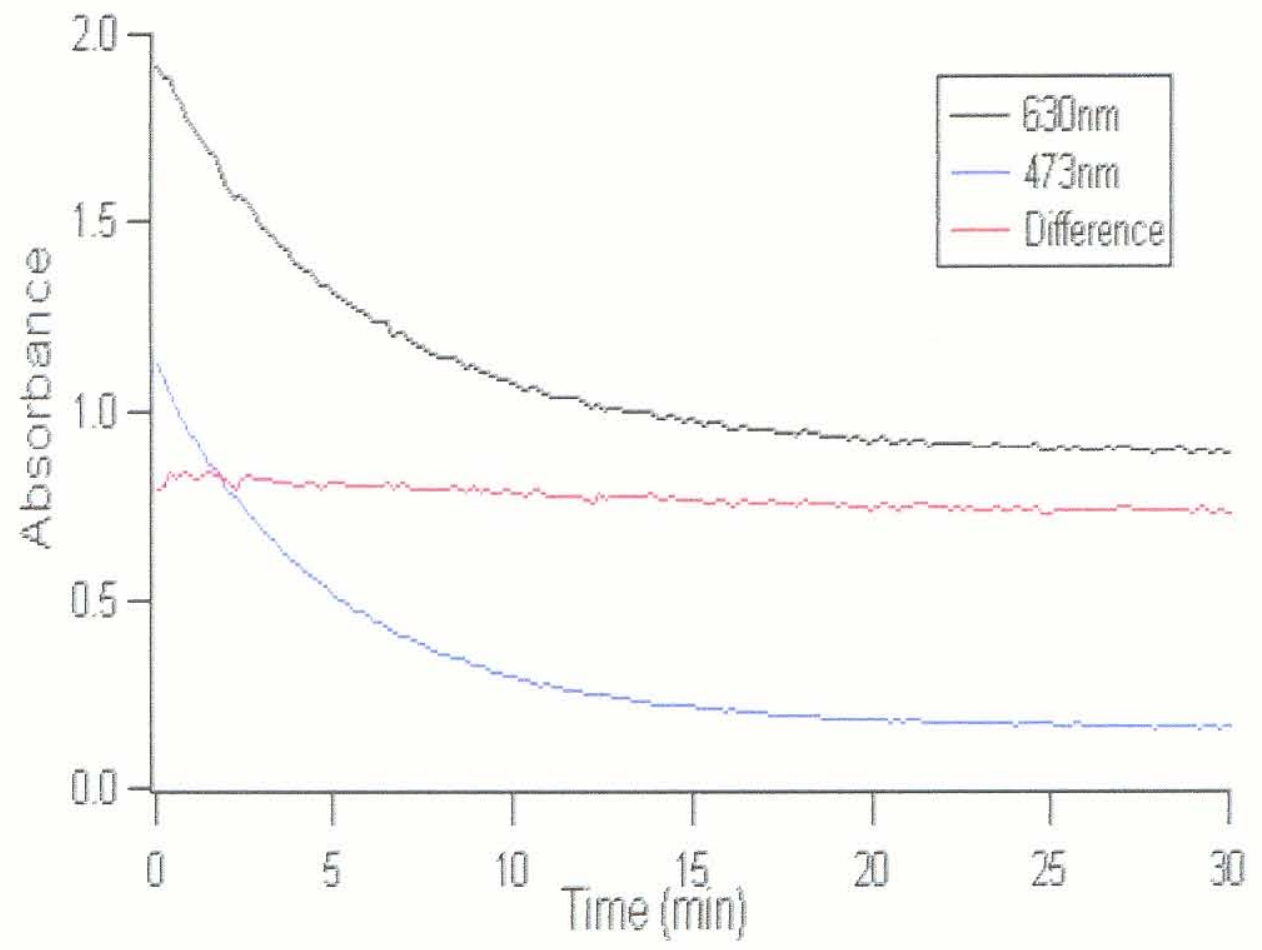

Figure 3-7: The difference in the absorbance at $473 \mathrm{~nm}$ and $630 \mathrm{~nm}$ is constant throughout the absorbance decay of malachite green.

The absorbance coefficients were obtained at $473 \mathrm{~nm}$ and $630 \mathrm{~nm}$ for phosphate standards ranging from 1-20 $\mu \mathrm{M}$. Both measurements showed excellent linearity. Most significantly, the difference between the absorbance at $630 \mathrm{~nm}$ and $473 \mathrm{~nm}$ displayed excellent correlation with the concentration of phosphate (Figure 3-8). 


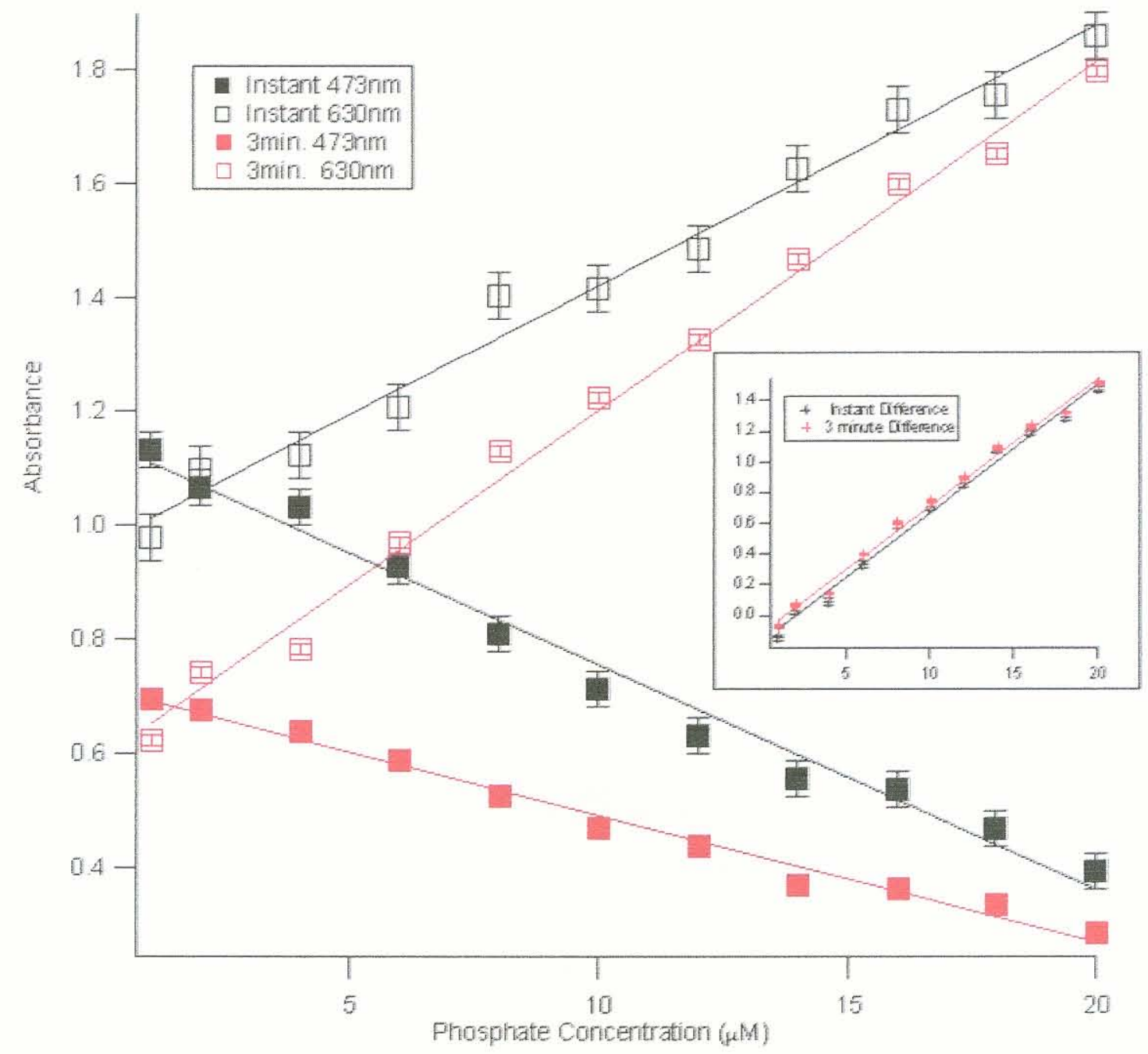

Figure: 3-8: Absorbance coefficients at $473 \mathrm{~nm}(\mathbf{\square}, \mathbf{m})$ also scale linearly with phosphate concentration. Inset: The difference between the absorbance measurements shows excellent correlation with phosphate concentration. Instant calibration (black) is comparable to 3 minute calibration (red).

While the absorbance coefficients obtained instantaneously are lower and show less precision, the difference between the absorbance used for the calibration proved to be as precise as the 3 minute calibration. The slope of the instantaneous calibration is comparable to the slope of 3 minutes ( 0.0842 and 0.830 respectively) indicating this truncated time scale does not lead to a loss in sensitivity. 


\subsection{Conclusions}

The malachite green phosphate analysis is ideally suited for archaeological prospectation. The method can easily be incorporated into in-stride analysis of remote locations because a limited amount of equipment is required for analysis and the developing reagents are robust. The instantaneous and the 3-minute method produced accurate quantitative results and allow for a more efficient survey of large sites than the methods previously published. However in order for the malachite green method to fully be integrated into soil analysis and to ensure the accuracy of the results, studies must be conducted to examine interferons to this method. 


\section{ARSENATE REDUCTION}

\subsection{Introduction}

It is imperative to examine potential interfering ions to the malachite green method in order to ensure the versatility of the phosphate analysis technique. A search of current literature reveals arsenate as an interferon to both the malachite green method and the molybdenum blue method of phosphate analysis (Carvalho et al. 1998; Dasgupta et al. 2002; Goulden and Brooksbank, 1974; Johnson, 1971; Linge and Oldham, 2001). In the case of malachite green, arsenate forms a complex with molybdate and then binds to malachite green. The formation of the arsenomolybdate and malachite green aggregate increases the absorbance, which in turn leads to inaccurate calculation of phosphate concentration. The molybdoarsenate complex is well studied and it is established that the reduced form of arsenate - arsenite - does not complex with molybdate (Carvalho et al. 1998; Dasgupta et al. 2002; Goulden and Brooksbank, 1974; Johnson, 1971; Linge and Oldham, 2001).

At $630 \mathrm{~nm}$ the molar absorptivity of arsenomolybdate-malachite green aggregate is between $1.80 \times 10^{5} \mathrm{M}^{-1} \mathrm{~cm}^{-1}$ and $2.9 \times 10^{5} \mathrm{M}^{-1} \mathrm{~cm}^{-1}$ (Matsubara et al. 1987; Takamura, 1992; Wang, 1993). This range coincides with the molar absorptivity of the phosphomolybdate-malachite green aggregate for the same wavelength $\left(2.6 \times 10^{5} \mathrm{M}^{-1} \mathrm{~cm}^{-1}\right.$ 
$-2.9 \times 10^{5} \mathrm{M}^{-1} \mathrm{~cm}^{-1}$ ) (Matsubara et al. 1994; Takamura, 1992; Wang, 1993). The overlap in absorption coefficient indicates that arsenate interferes with the phosphate analysis.

The interference is clearly seen by comparing the spectrum produced by an arsenate and a phosphate solution (Figure 4-1). Both ions form aggregates with malachite green and produce spectra that are nearly indistinguishable between 500-700 nm.

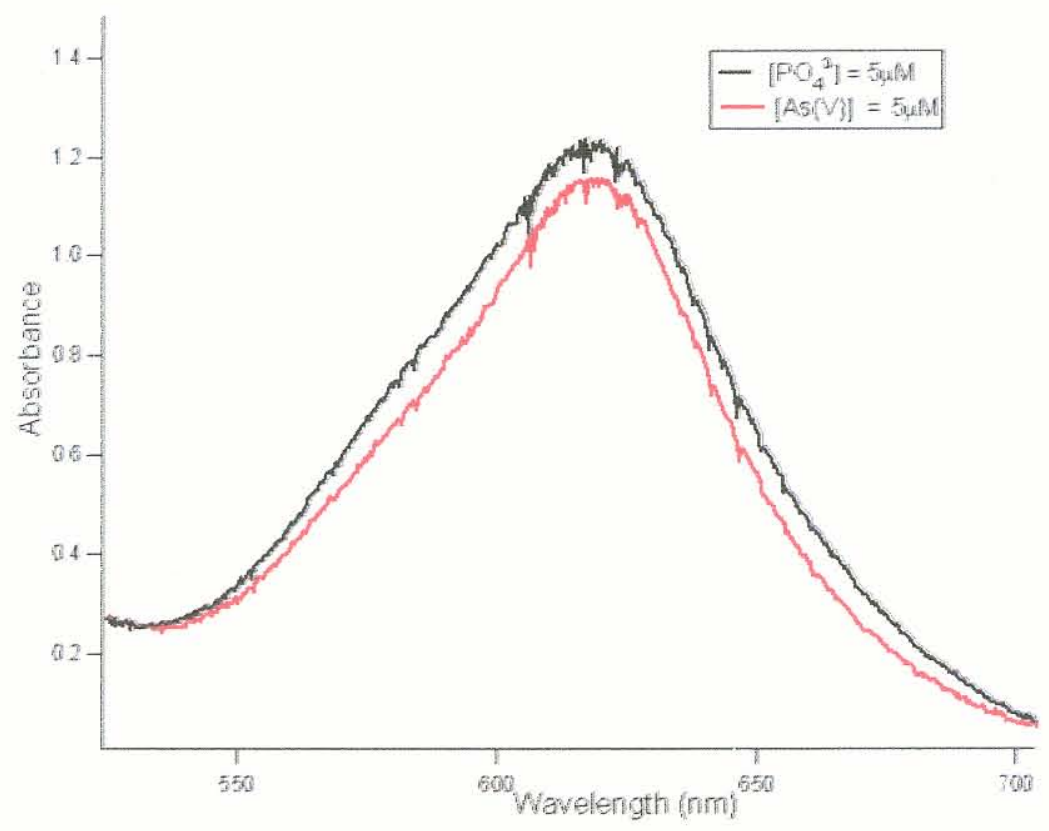

Figure 4-1: Both the phosphomolybdate-malachite green and arsenomolybdate-malachite green complexes have a maximum absorbance at $630 \mathrm{~nm}$.

As a result of the similarities of the absorbance of phosphomolybdate-malachite green and the arsenomolybdate complexes, when arsenate is added to a phosphate solution the total absorbance due to bound malachite green increases (Figure 4-2). 


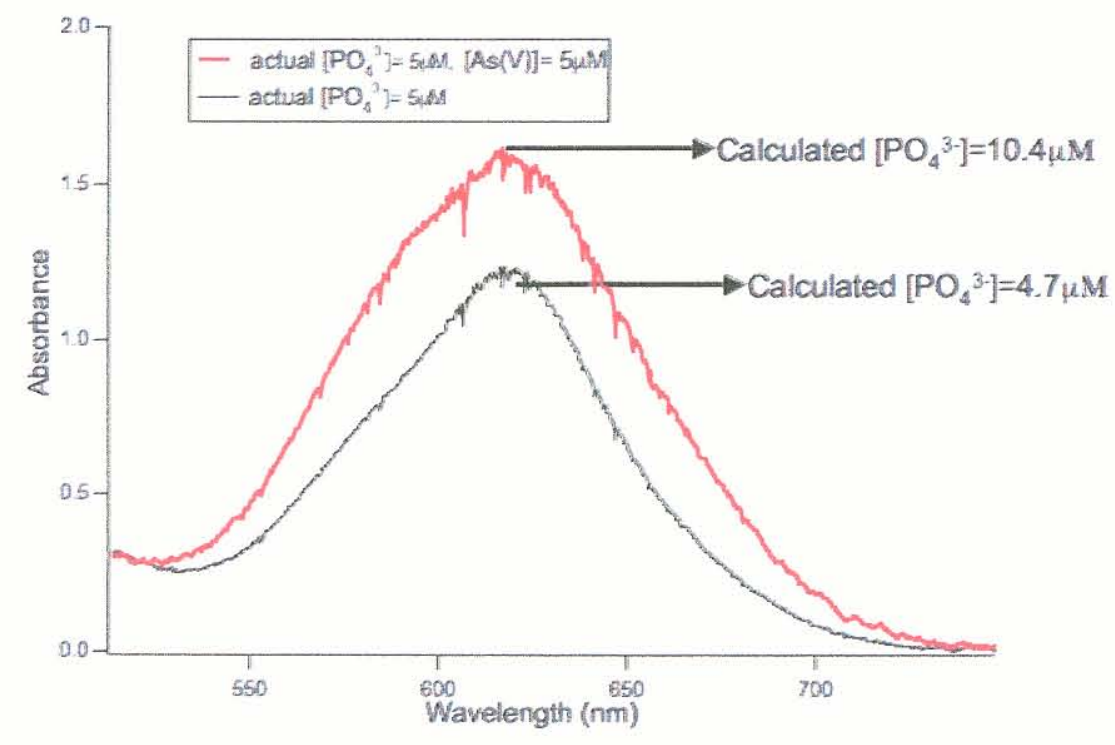

Figure 4-2: $\mathrm{As}(\mathrm{V})$ increases the absorbance and causes inaccuracies in phosphate concentration calculation. $\left[\mathrm{PO}_{4}{ }^{3-}\right]$ was calculated using the phosphate only calibration.

It is clear from Figure 4-2 that the presence of arsenate will result in inaccurate phosphate analysis. The error is even more significant in archaeological soil extracts, which are often diluted by factors of fifty or greater. The dilution amplifies the error in the final calculation of phosphate. For example, if the sample shown above in Figure 4-2 spiked is scaled for a dilution of 50 , the final phosphate concentration of the spiked sample is $520 \mu \mathrm{M}$; as opposed to the accurate concentration of $235 \mu \mathrm{M}$. When compared with other samples for a large site, the over estimation may result in a mislabeling of "high" phosphate concentration and result in the false identification of an anthropic soil. Therefore, for accurate phosphate analysis, the arsenate interference must be eliminated. 
Although arsenate is not a species typically found in soil as a major component, it is possible for this ion to interfere with soil phosphate analysis. The interference does not result from the native $\mathrm{As}(\mathrm{V})$ since native concentrations are low. Rather, the interference results from the addition of As from anthropogenic activities such as mining, disposal of industrial waste and use of pesticides. For example, As concentrations in the vicinity of a mining area have been reported as $27,000 \mathrm{mg} \mathrm{kg}^{-1}$ of soil (Frank, 2005). An arsenic concentration of this magnitude undoubtedly interferes with the malachite green method.

Soil samples tainted with arsenic must be treated with an arsenate reducing agent to ensure accurate phosphate analysis. Thiosulfate and L-Cysteine have been employed in the literature for arsenate reduction in soil and water samples. The methodology used for both reducing agents varies greatly between authors (Carvalho et al. 1998; Dasgupta et al. 2002; Goulden and Brooksbank, 1974; Johnson, 1971; Linge and Oldham, 2001). Table 4-1 gives a brief overview of the most important aspects of the procedures for both reagents.

\begin{tabular}{|c|c|c|c|c|}
\hline $\begin{array}{l}\text { Reducing } \\
\text { reagents }\end{array}$ & $\begin{array}{c}\text { Phosphate } \\
\text { Analysis } \\
\text { Method }\end{array}$ & $\begin{array}{l}\text { Pre-reduction } \\
\text { Time (minutes) }\end{array}$ & $\begin{array}{c}\text { Additional } \\
\text { Reagents/ } \\
\text { condsiderations }\end{array}$ & Reference \\
\hline Thiosulfate & $\begin{array}{l}\text { Ascorbic } \\
\text { Acid }\end{array}$ & 8 & Metabisulfite & $\begin{array}{c}\text { Goulden and } \\
\text { Brooksbank, } 1974\end{array}$ \\
\hline Thiosulfate & $\begin{array}{l}\text { Ascorbic } \\
\text { Acid }\end{array}$ & 15 & Metabisulfite & Johnson, 1971 \\
\hline Sulfite & $\begin{array}{l}\text { Malachite } \\
\text { Green }\end{array}$ & 60 & 一 & $\begin{array}{l}\text { Linge and Oldham, } \\
2001\end{array}$ \\
\hline L-Cysteine & $\begin{array}{l}\text { Ascorbic } \\
\text { Acid }\end{array}$ & 5 & $\begin{array}{l}\text { Heat Sample to } \\
\qquad 80^{\circ} \mathrm{C}\end{array}$ & $\begin{array}{c}\text { Carvalho et al., } \\
1998\end{array}$ \\
\hline L-Cysteine & $\begin{array}{l}\text { Ascorbic } \\
\text { Acid }\end{array}$ & 5 & $\begin{array}{l}\text { Heat Sample to } \\
\qquad 75^{\circ} \mathrm{C}\end{array}$ & $\begin{array}{c}\text { Dasgupta et al ,, } \\
2002\end{array}$ \\
\hline
\end{tabular}

Table 4-1: Summary of arsenate reduction methods 
The methods listed in the above table are suitable for laboratory analysis but are non-optimal for in-stride analysis. For example although the 5-minute pre-reduction time required for L-Cysteine is ideal for large-scale archaeological surveys, the sample must be heated. This requirement is not conducive for fieldwork in remote locations. If the temperature is not elevated above room temperature, L-Cysteine requires an hour to completely reduce $\mathrm{As}(\mathrm{V})$, which also overly time-consuming.

The time requirement of thiosulfate pre-reduction is appropriate for a large scale, in-situ phosphate prospection. However, this technique is often cited as creating additional interference to phosphate analysis. In acidic solutions, thiosulfate decomposes to form colloidal sulfur. The colloid increases the turbidity of the sample and interferes with the absorbance measurements of the solution (Carvalho et al. 1998; Goulden and Brooksbank, 1974; Johnson, 1971; Linge and Oldham, 2001). Linge et al. (2001) combined the thiosulfate method of reduction with the malachite green phosphate analysis, and found that colloidal sulfur formed immediately upon the addition of malachite green. Subsequently, the group eliminated the possibility of using thiosulfate in combination with malachite green.

Utilizing the published methodology, neither reduction method is suitable for instride malachite green phosphate analysis. The compatibility of both methods with the malachite green must be investigated. Before L-Cysteine can be integrated into fieldwork, the reduction time must be shortened. The thiosulfate method must be altered such that colloidal sulfur does not form when the malachite green method is used. To accomplish these goals, a series of methodological modification trials were conducted. 


\subsection{Reagents}

Stock solutions of phosphate and arsenate were prepared from $\mathrm{KH}_{2} \mathrm{PO}_{4}$ (Aldrich, $99 \%$ ) and $\mathrm{Na}_{2} \mathrm{HAsO}_{4}$ (Aldrich, 98\%), respectively, without further purification. Working solutions were prepared by volumetric dilution.

A $250 \mathrm{mM}$ L-Cysteine (Aldrich, 97\%) reducing agent was prepared daily in $0.3 \mathrm{M}$ $\mathrm{HCl}$. For thiosulfate reduction, a stock solution of $20 \mathrm{mM}$ thiosulfate was prepared from $\mathrm{Na}_{2} \mathrm{~S}_{2} \mathrm{O}_{3}$ (Aldrich, 99\%).

\subsection{Kinetic Effects of Arsenate on Phosphate Analysis}

Although the phosphate and $\mathrm{As}(\mathrm{V})$ complexes have indistinguishable absorbance spectra, kinetic analysis shows that these two species behave differently after the addition of malachite green reagents (Figure 4-3). 


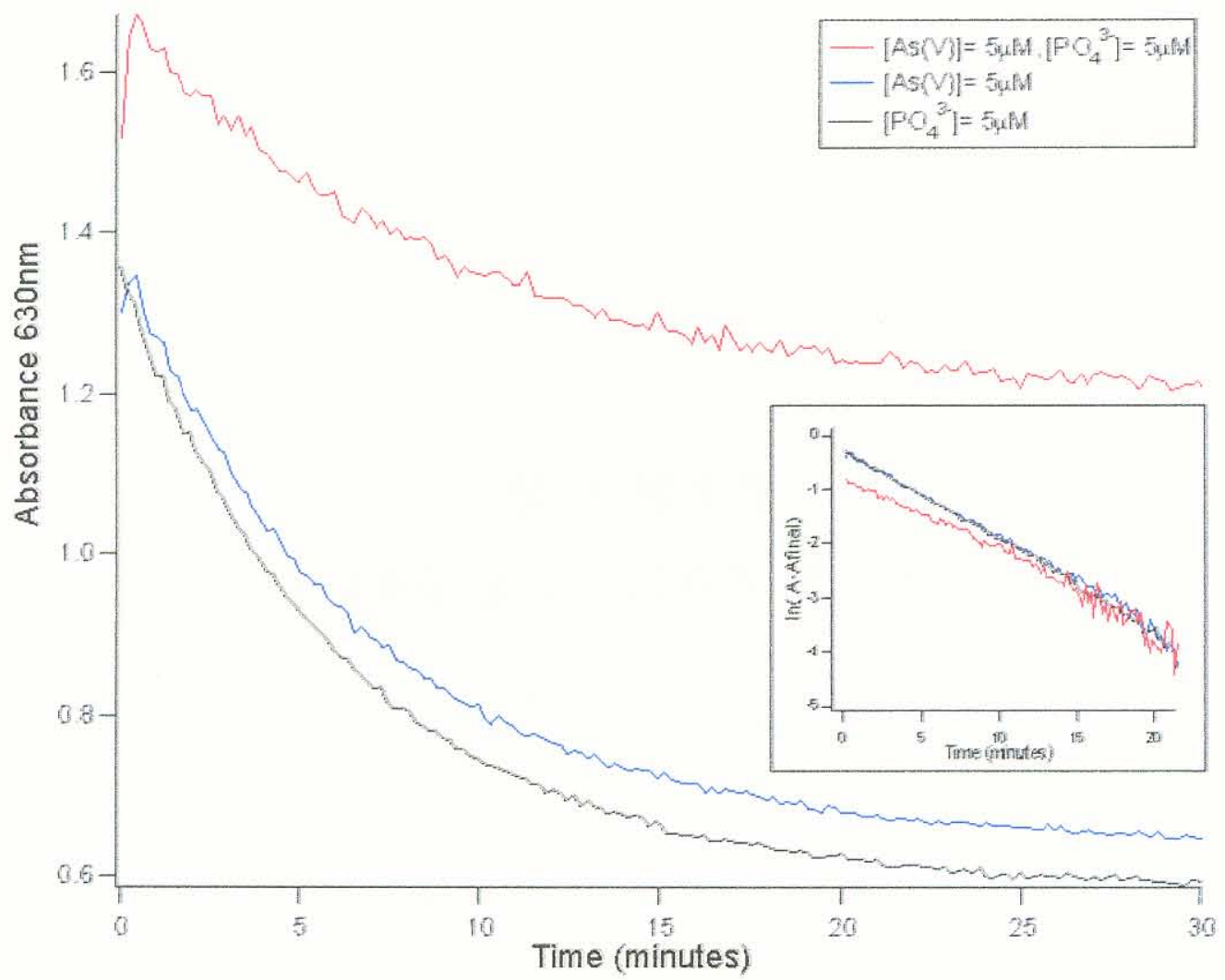

Figure 4-3: A comparison of the kinetics at 630nm of As(V), phosphate, and mixed solutions shows differing absorbance decays during the malachite green development process. Linear decays (inset) confirm the reduction process of malachite green is altered in the presence of $\mathrm{As}(\mathrm{V})$.

In an $\mathrm{As}(\mathrm{V})$ solution the first several seconds are characterized by a rapid increase in absorbance resulting from a shift in the form of malachite green upon addition to the As(V) solution. When the malachite green solution is added to the As(V), a small fraction of the dye is converted to the yellow form. The increase in absorbance is the result of the shift back to the green form of malachite green. The rapid shift back to malachite green causes a steep decline in absorbance for the first minute of the $473 \mathrm{~nm}$ decay (Figure 4-4). 


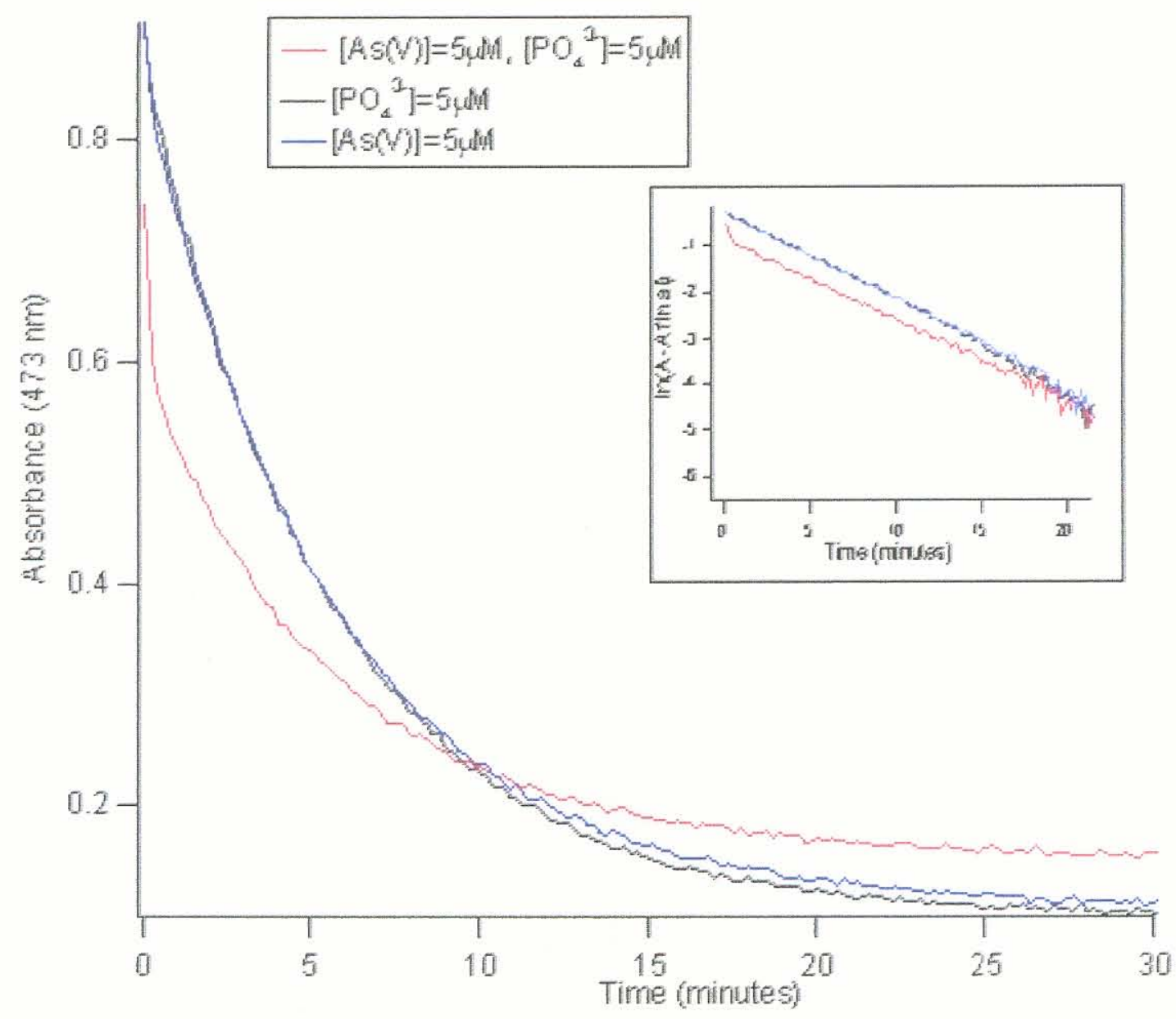

Figure 4-4: The absorbance decay of $\mathrm{As}(\mathrm{V})$ and phosphate solutions displays a rapid decrease in absorbance for the first minute as the yellow malachite green is converted to the green form. Linear kinetic decays (inset) confirm the change in reduction rate of the unbound malachite green at $473 \mathrm{~nm}$ in solutions containing both $\mathrm{As}(\mathrm{V})$ and phosphate.

Despite the initial disparity, analysis of the linear kinetic decay shows that the reduction of the unbound malachite green in an $\mathrm{As}(\mathrm{V})$ solution is comparable to a phosphate only solution. Solutions containing both $\mathrm{As}(\mathrm{V})$ and phosphate also exhibit an increase in initial absorbance. Again, the increase occurs as the protonated malachite green converts back to the green form. In the case of the mixed solution, the reduction of the unbound malachite green is affected. The slope of the linear absorbance decay for a 
mixed solution differs from the phosphate only solution, which indicates the rate has been altered by the presence of $\operatorname{As}(\mathrm{V})$. The rapid rise in absorbance is evidence that the reduction kinetics are atypical and serves as a means of alerting the experimenter that an interferon is present. However in order to ensure accurate calculated phosphate concentration, $\mathrm{As}(\mathrm{V})$ must be reduced to $\mathrm{As}(\mathrm{III})$.

\subsection{L-Cysteine Reduction of Arsenate}

In the published methods, the concentration of L-Cysteine varies greatly with respect to the concentration of $\mathrm{As}(\mathrm{V})$. The concentration of 1 -Cysteine must be carefully determined because it is known that excessive amounts of L-Cysteine hinder the reduction process. For this work, the amount of L-Cysteine was varied in order to determine the optimal concentration for reduction of solutions containing $26 \mu \mathrm{M} A s(V)$. Before the solution was analyzed with malachite green, it was allowed to stand for one hour to ensure complete reduction (Figure 4-5). 


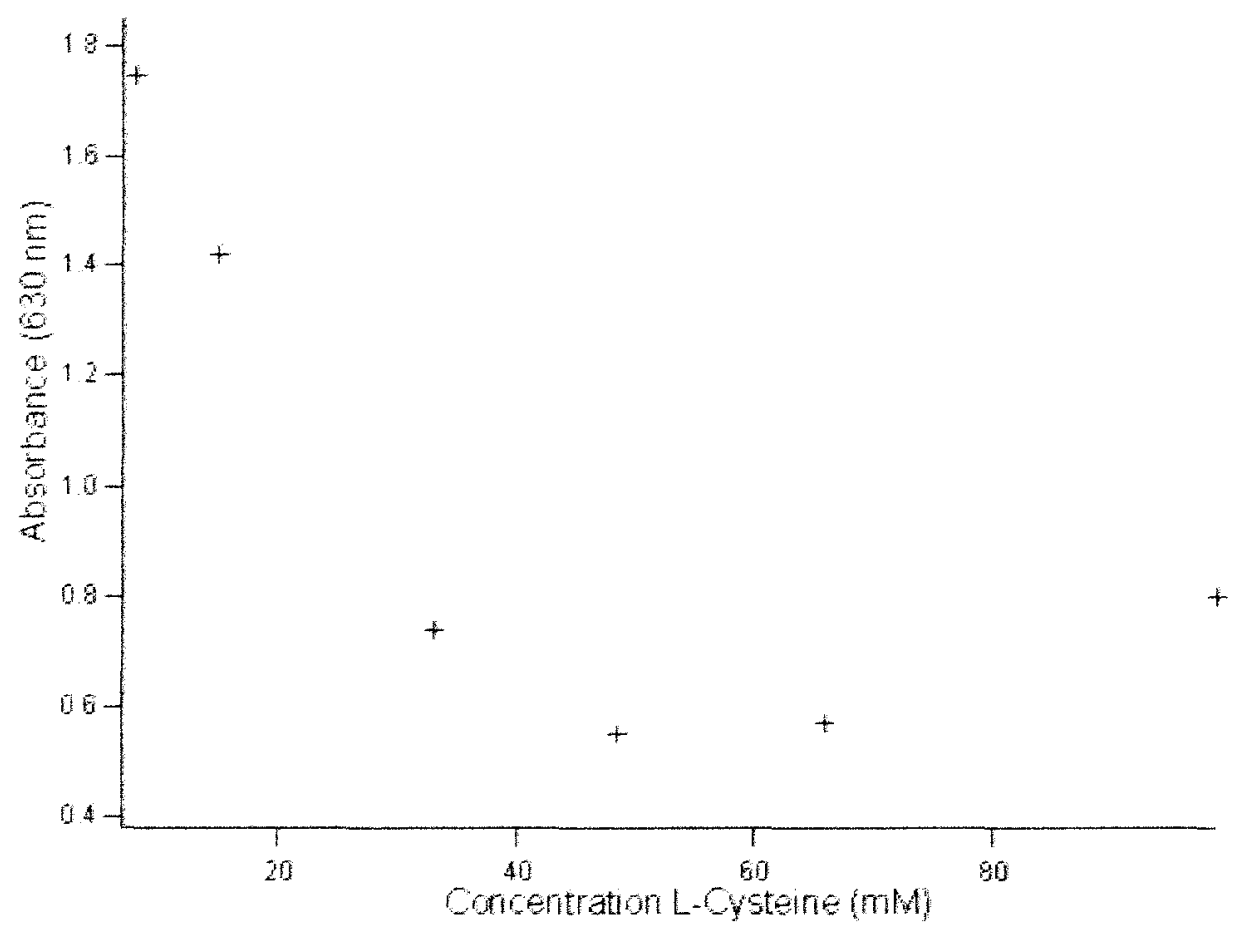

Figure 4-5: Standards containing $26 \mu \mathrm{M} A s(\mathrm{~V})$ were reduced with varied concentrations of L-Cysteine. Samples with a final L-Cysteine concentration between $48.5 \mathrm{mM}-65$ $\mathrm{mM}$ showed greatest reduction in signal.

$\mathrm{As}(\mathrm{III})$ is incapable of forming the complex with malachite green that absorbs at $630 \mathrm{~nm}$. Therefore, solutions displaying low absorbance values have the greatest reduction of $\mathrm{As}(\mathrm{V})$. The lowest absorbance occurred in solutions with a final L-Cysteine concentration between $48.5 \mathrm{mM}$ and $65 \mathrm{mM}$, which indicates this concentration range is ideal for reduction of $\mathrm{As}(\mathrm{V})$. To achieve a final concentration of $62 \mathrm{mM}$ L-Cysteine, 1 $\mathrm{mL}$ of $25 \mathrm{mM}$ stock solution added to $3 \mathrm{~mL}$ of sample.

As should be evident from previous discussions, an hour-long reduction period is unsuitable for a large-scale field survey. In order to gain an understanding of the time requirements of L-Cysteine reduction, $10 \mu \mathrm{M} \mathrm{As}(\mathrm{V})$ solutions were reduced, and the time allotted for reduction was varied (Figure 4-6). Reduction times greater than 20 minutes did not consistently show a large increase in the reduction of the arsenate signal. Again, 
it is important to remember that for an archaeological survey, the slight inaccuracies caused by incomplete reduction do not justify an additional 40 minutes for complete reduction.

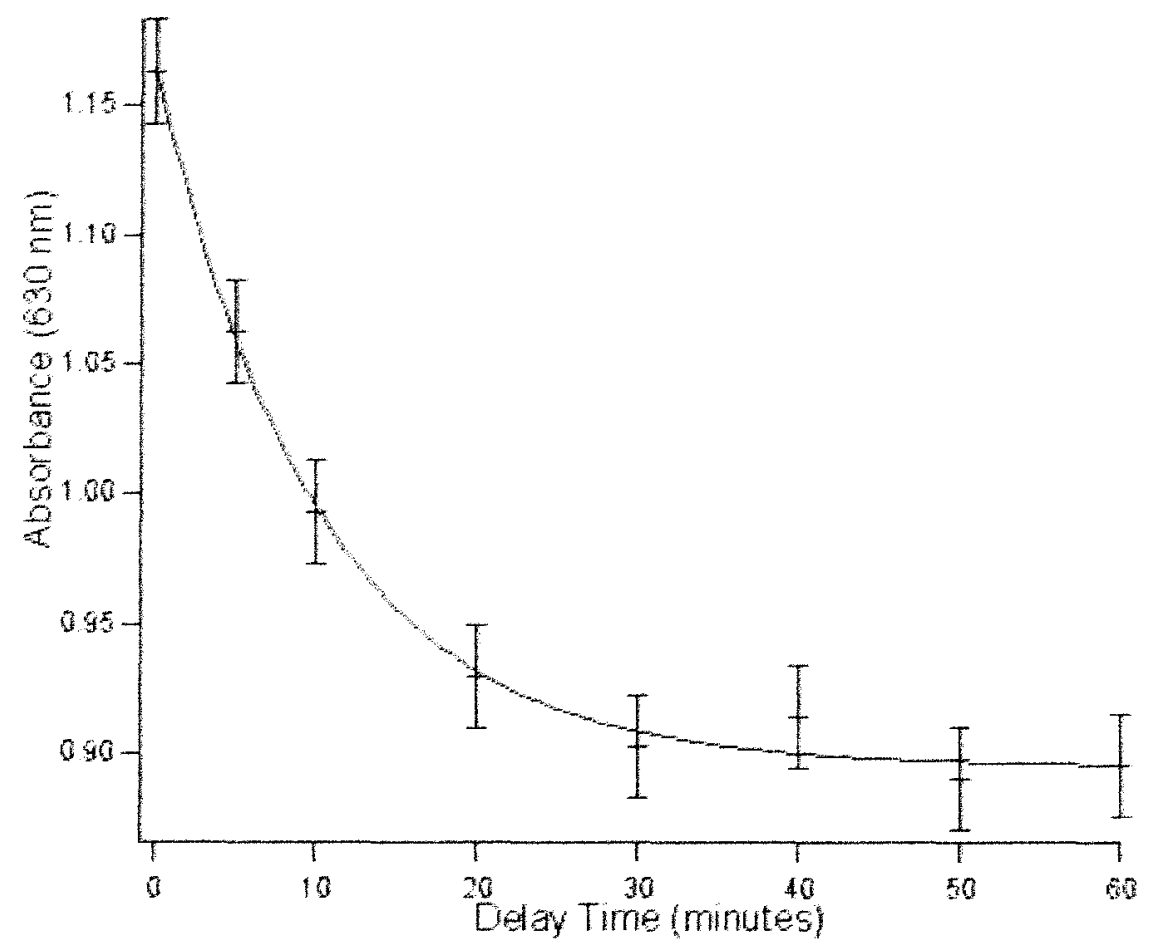

Figure 4-6: Time dependence of L-Cysteine reduction. A solution of $5 \mu \mathrm{M} \mathrm{PO}_{4}{ }^{-3}$ and 10 $\mu \mathrm{M} A s(\mathrm{~V})$ was reduced for varied amount time. 20 minute reduction completely reduced $\mathrm{As}(\mathrm{V})$.

Kinetic analysis showed that although the absorbance is lowered by treatment with L-Cysteine, the development of malachite green is unaffected (Figure 4-7). Both the unreduced and reduced solutions display similar absorbance decays over the 30 minute time period. The slopes of the linear absorbance decays are parallel indicating that the rate of the reduction of the unbound malachite green is unchanged. 


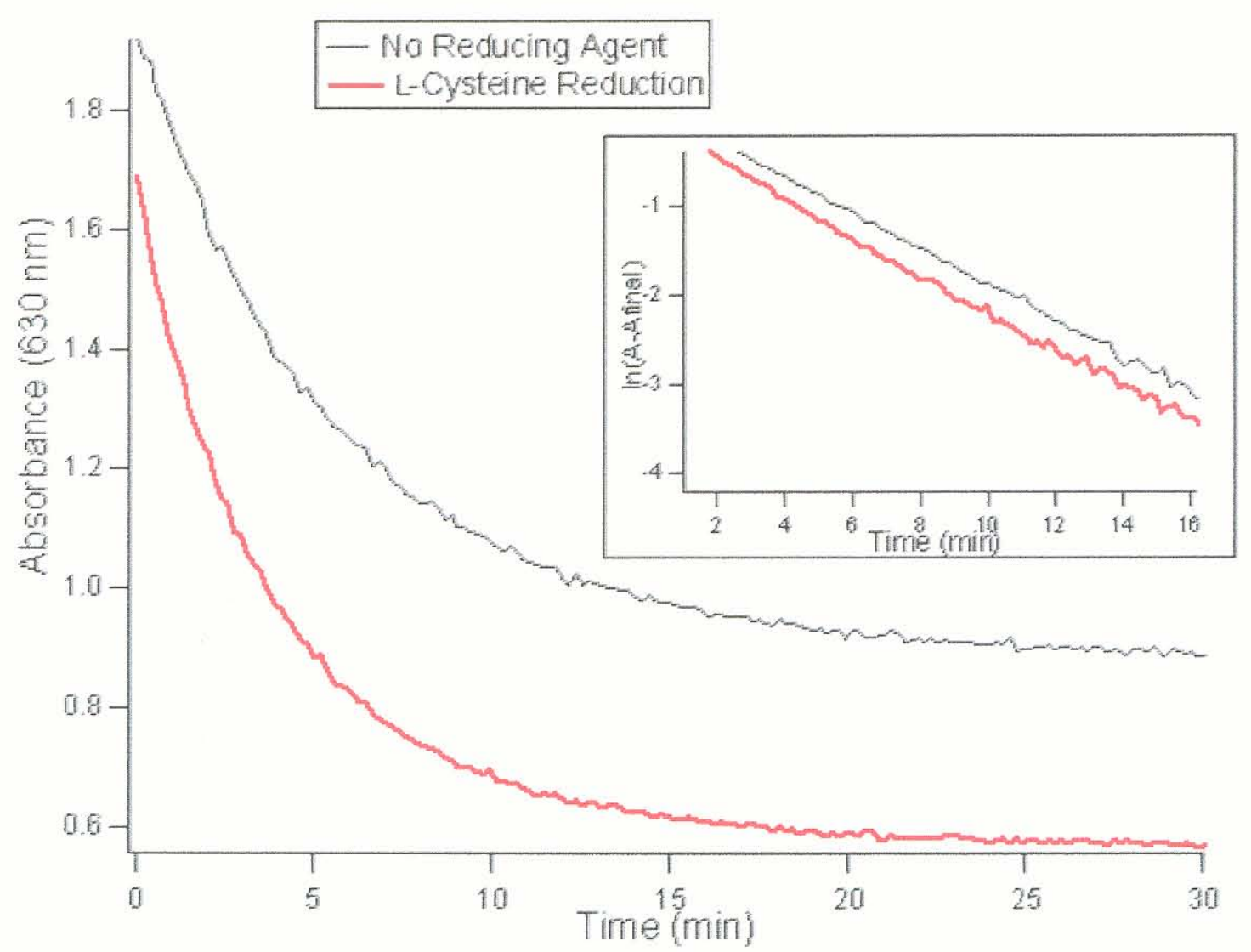

Figure 4-7: Kinetic analysis at $630 \mathrm{~nm}$ shows that pre-reduction with L-Cysteine does not alter the absorbance decay. The slopes of the linear decays (inset) are parallel indicating the reduction rate of unbound malachite green is not affected.

The L-Cysteine method was used on phosphate standards to create a calibration (Figure 4-8). The resulting calibration implies that reduction with L-Cysteine does not result in decreased sensitivity or diminished reproducibility. The resulting absorbance data show excellent correlation with phosphate concentration and the resulting regression value is 0.999 . The slope of the L-Cysteine calibration is 0.0585 , which is in agreement with the slope of the calibration of a standard development (0.0656). The deviations in absorbance measurements are consistent with the deviations associated with samples that 
are unreduced. L-Cysteine can be used in combination with malachite green analysis without adversely affecting the phosphate calculation.

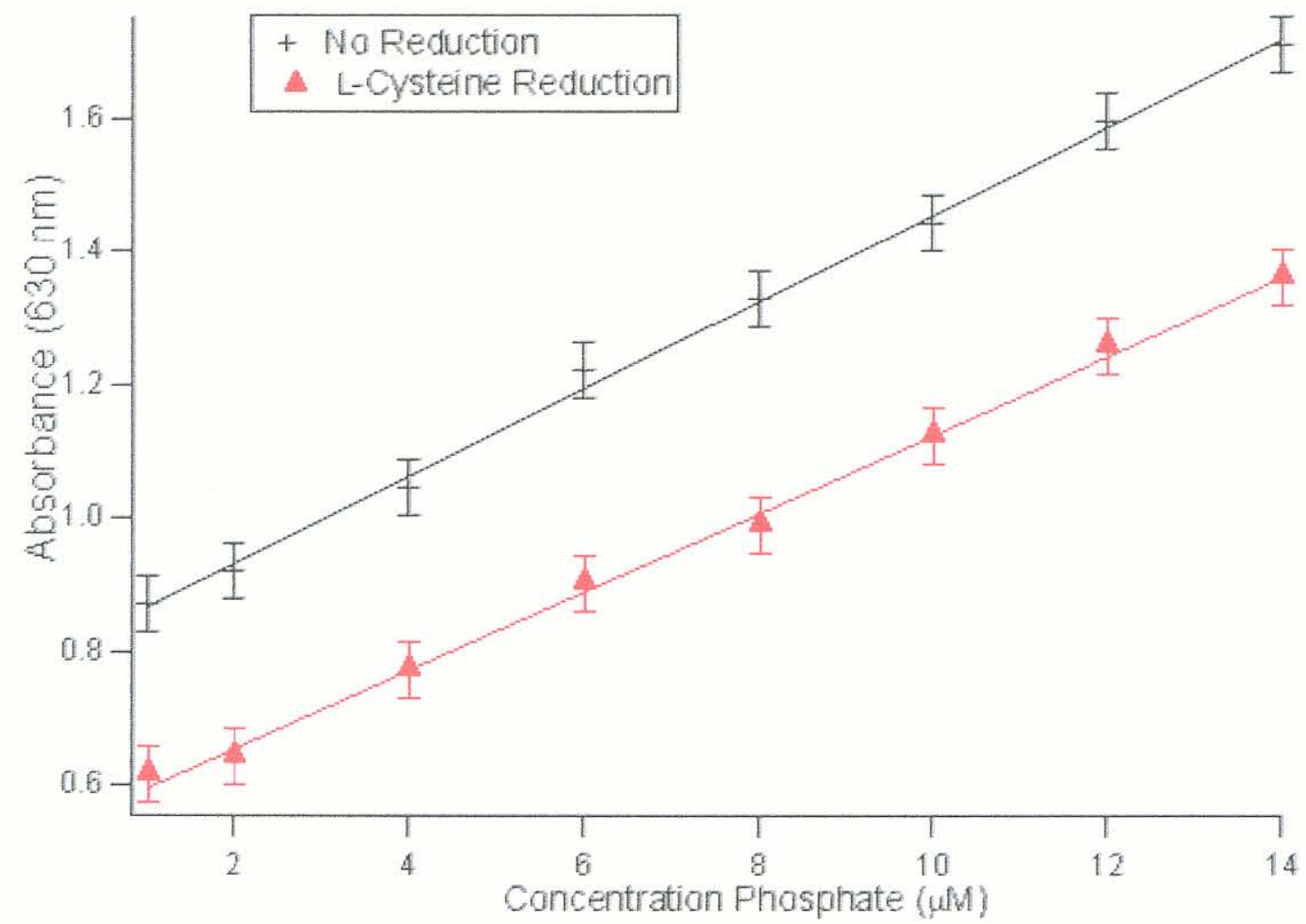

Figure 4-8: L-Cysteine pre-reduction does not lead to a decrease in sensitivity and reproducibility, or diminished correlation.

\subsection{Thiosulfate Reduction of Arsenate}

For thiosulfate reduction, $3 \mathrm{~mL}$ of standard solution were mixed with $0.5 \mathrm{~mL}$ of $0.45 \mathrm{M} \mathrm{H}_{2} \mathrm{SO}_{4}$ and $0.3 \mathrm{~mL}$ of $20 \mathrm{mM}$ sodium thiosulfate. The concentration of sodium thiosulfate is consistent with the concentration range of the previously published methods (Goulden and Brooksbank, 1974; Johnson, 1971). However, the molarity of the sulfuric acid is significantly lower to accommodate the $\mathrm{pH}$ sensitive malachite green development. The lower concentration of sulfuric acid also decreases the impact of environmental contamination in the event of a spill during in-stride analysis. Unlike the 
published methods, metabisulfite was not added to the reducing reagents to prevent the precipitation of colloidal sulfur. Although metabisulfite was not included, evidence of colloidal sulfur and interference to the absorbance were not observed during the period of reduction or phosphate analysis. The lack of colloidal sulfur can be attributed to both the decrease in the concentration of sulfuric acid and the truncated time of the malachite green development. As the acidity of the solution increases, the formation of sulfur increases. In this case, the reduced samples do not become visibly turbid until an hour after $0.45 \mathrm{M} \mathrm{H}_{2} \mathrm{SO}_{4}$ and $20 \mathrm{mM}$ sodium thiosulfate are added. Therefore, because the absorbance is recorded a maximum of 3 minutes after the addition of malachite green the signal is not affected.

In the literature, the time allotted for reduction varied from 8 minutes to one hour. Reducing agents were added to a $10 \mu \mathrm{M} \mathrm{As}(\mathrm{V})$ solution and the time delay before adding the malachite green reagents was varied in order to determine the optimal reduction time (Figure 4-9).

A 5-minute delay greatly decreased the absorbance when compared to the absorbance of solutions with instantaneous addition of developing reagents. Delay times greater than 5 minutes did not show a significant decrease in absorbance. Although the reduction is not complete at 5 minutes, for archaeological purposes the slight inaccuracies introduced do not justify the additional 25 minutes. The shorter reduction period allows the analysis of a greater number of samples and is well suited for large-scale surveys. Complete reduction is achieved after 10 minutes. The reduction period can be extended to 10 minutes if accuracy is the primary concern as opposed to time. 


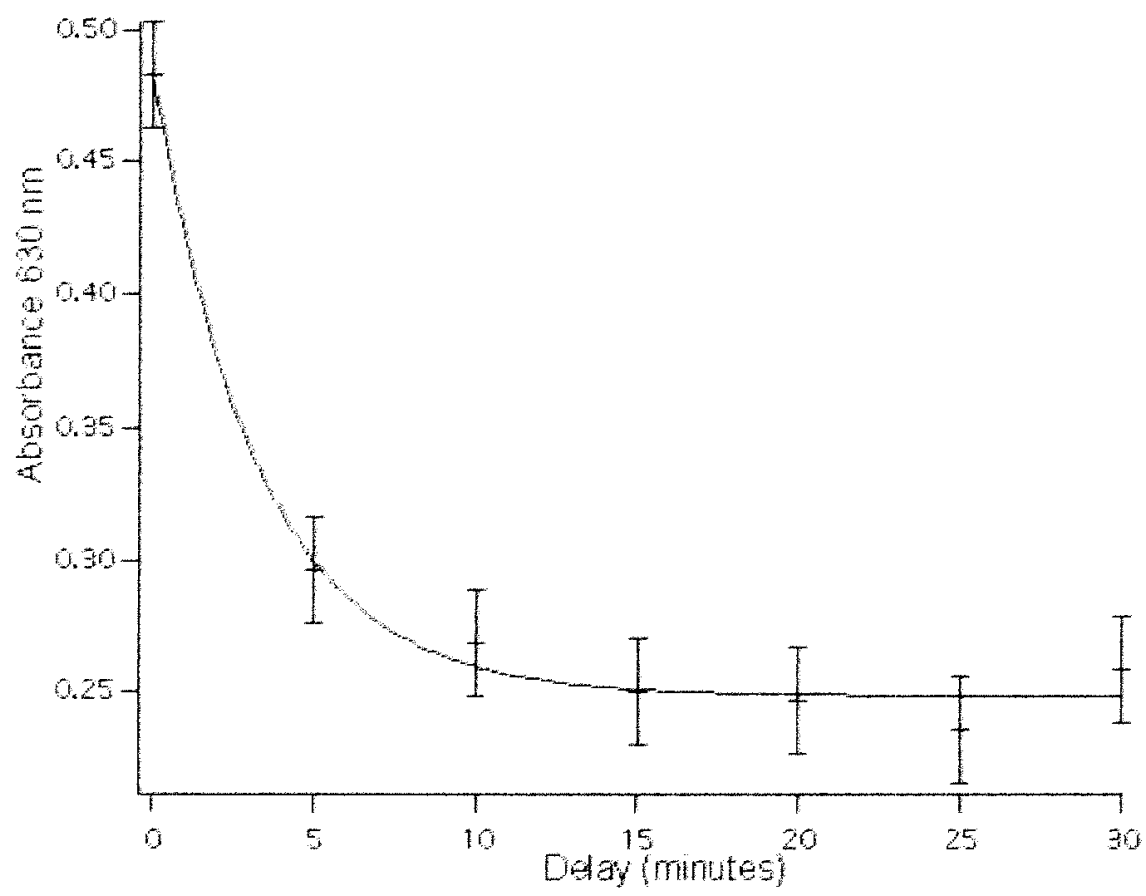

Figure 4-9: The delay time was varied to determine the time required to reduce a $10 \mu \mathrm{M}$ $\mathrm{As}(\mathrm{V})$ solution with thiosulfate. A 5 minute delay time sufficiently reduced the interference of $\mathrm{As}(\mathrm{V})$.

The previously published methods also have differing methodology for the addition of the reducing reagents. Goulden and Brooksbank (1974) add the sulfuric acid and the thiosulfate to the arsenate solution separately. Johnson (1971) premixes the sulfuric acid and thiosulfate before addition to the arsenate solution. To determine which methodology was more suitable, standard solutions of phosphate- ranging from $0 \mu \mathrm{M}$ $17 \mu \mathrm{M}$ - were treated with thiosulfate and sulfuric acid added separately and as a premixed solution. When the thiosulfate and sulfuric acid reducing reagents were added separately, there was an increased correlation in the calibration over the standards treated with premixed reagents (Figure 4-10). Premixed reagents have a regression value of 0.897 , whereas the separate addition of the reagents increased the regression to 0.992 . 


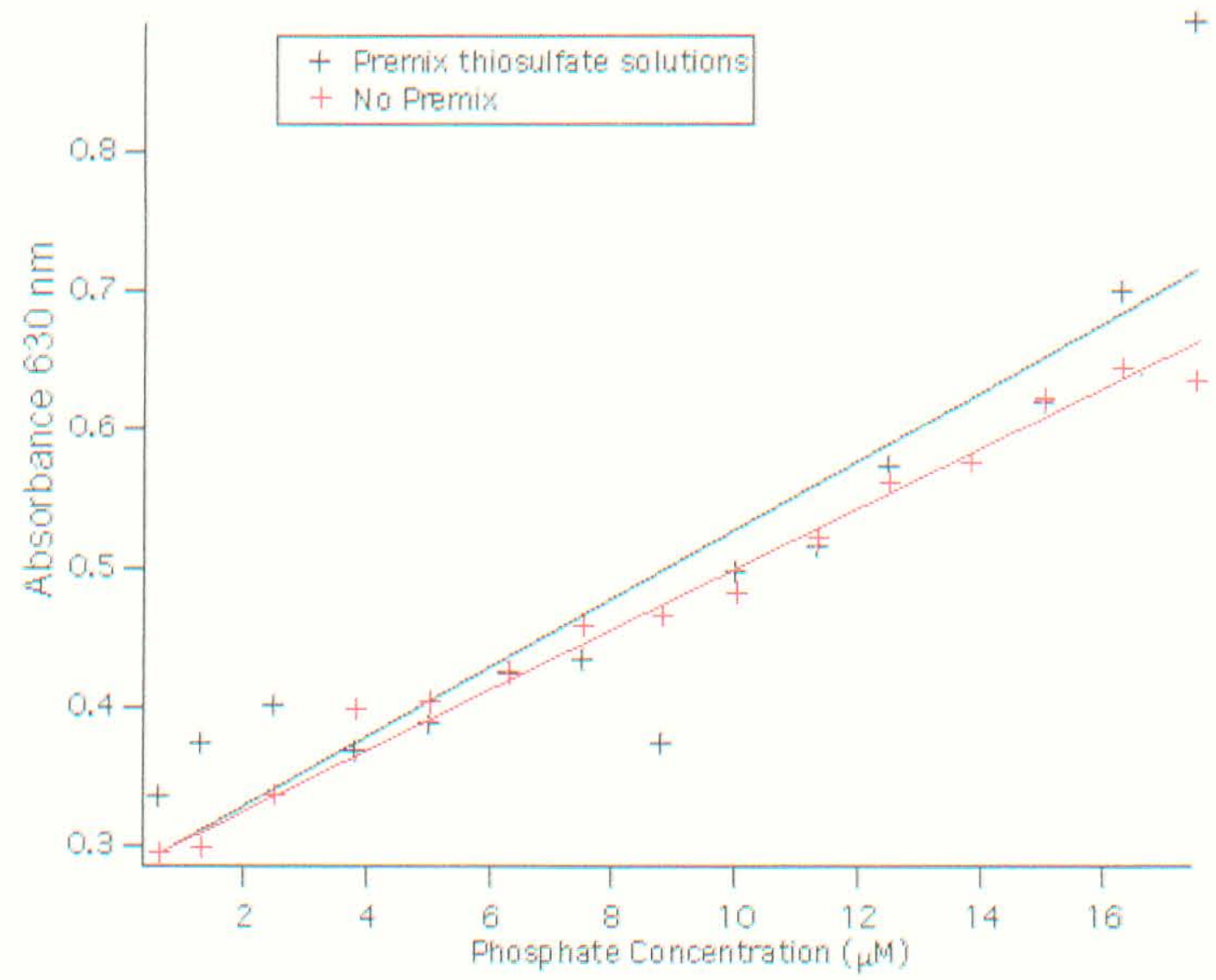

Figure 4-10: A calibration made by separate addition of thiosulfate reducing reagents shows an increase correlation. The regression values are 0.992 and 0.897 respectively.

The increased correlation between absorbance and concentration supports separate addition of the reagents. It is also noteworthy that the pre-mixed solution is only stable for 24 hours. If sodium thiosulfate and sulfuric acid are kept separate the solutions are stable indefinitely, which is ideal for archaeological surveys in remote locations.

Kinetic analysis at $630 \mathrm{~nm}$ shows that thiosulfate pre-reduction alters the development of malachite green (Figure 4-11). 


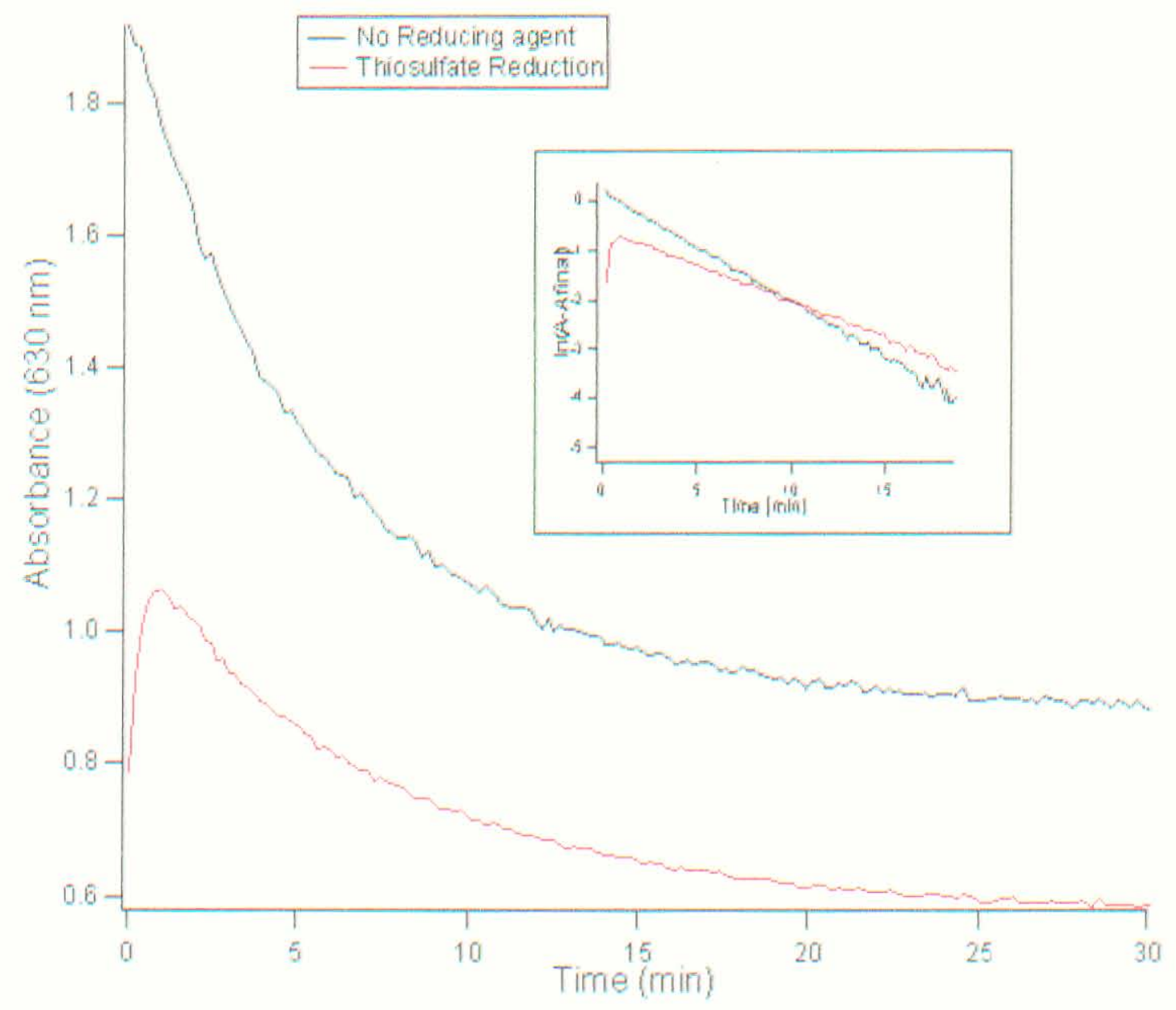

Figure 4-11: Kinetic Analysis of Thiosulfate Pre-reduction at $630 \mathrm{~nm}$. Standard solution of $10 \mu \mathrm{M}$ phosphate pre-reduced with thiosulfate shows altered malachite green development. (Inset) Dissimilarities in slope of linear kinetic decay confirm there is a change in reduction process of malachite green.

In the case of pre-reduced samples, there is an increase in absorbance rather than a decrease for the first minute of development. Also, the linear kinetic decay of the prereduced solution has a noticeably different slope than that of the unreduced solution. It can be concluded the incorporation of thiosulfate pre-reduction slows the rate of reduction of the unbound malachite green.

The change in behavior occurs because the malachite green is reduced immediately after it is added to the reduced solution. The luecomalachite green 
associates with phosphate and is then oxidized back to the green form, and as a result there is a rapid rise in the $630 \mathrm{~nm}$ absorbance. Figure 4-12 shows that the absorbance at $473 \mathrm{~nm}$ is also altered during the initial moments of development. There is a change in the rate, which indicates that the yellow/green equilibrium is also affected by the additional $\mathrm{H}_{2} \mathrm{SO}_{4}$.

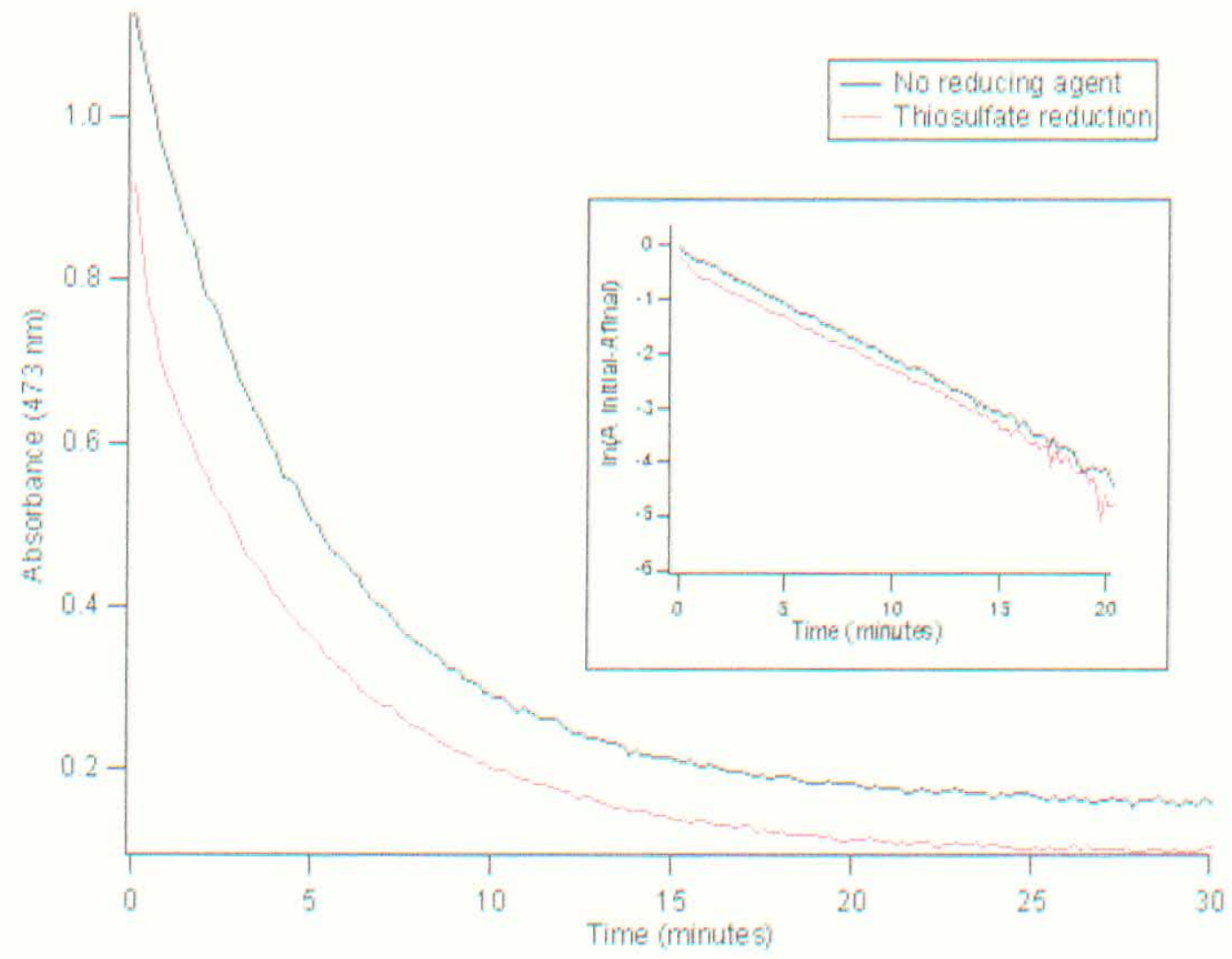

Figure 4-12: Kinetic analysis of thiosulfate pre-reduction at $473 \mathrm{~nm}$ show the yellow form of malachite green is altered by the additional $\mathrm{H}_{2} \mathrm{SO}_{4}$.

Although the development of malachite green is altered by the addition of thiosulfate reagents, a calibration was created using phosphate standards pre-reduced with thiosulfate. Figure 4-13 shows the resulting calibration has excellent correlation $(\mathrm{r}=$ 0.999) and precision. The slope of the reduced phosphate solutions is slightly less that 
the untreated calibration ( 0.0527 and 0.0665 , respectively), which indicates a slight loss in sensitivity. The minimal loss is acceptable within the confines of a relative phosphate survey. Sodium thiosulfate displays excellent potential for archaeological fieldwork because it effectively reduces $\mathrm{As}(\mathrm{V})$ to $\mathrm{As}(\mathrm{III})$ within 5 minutes.

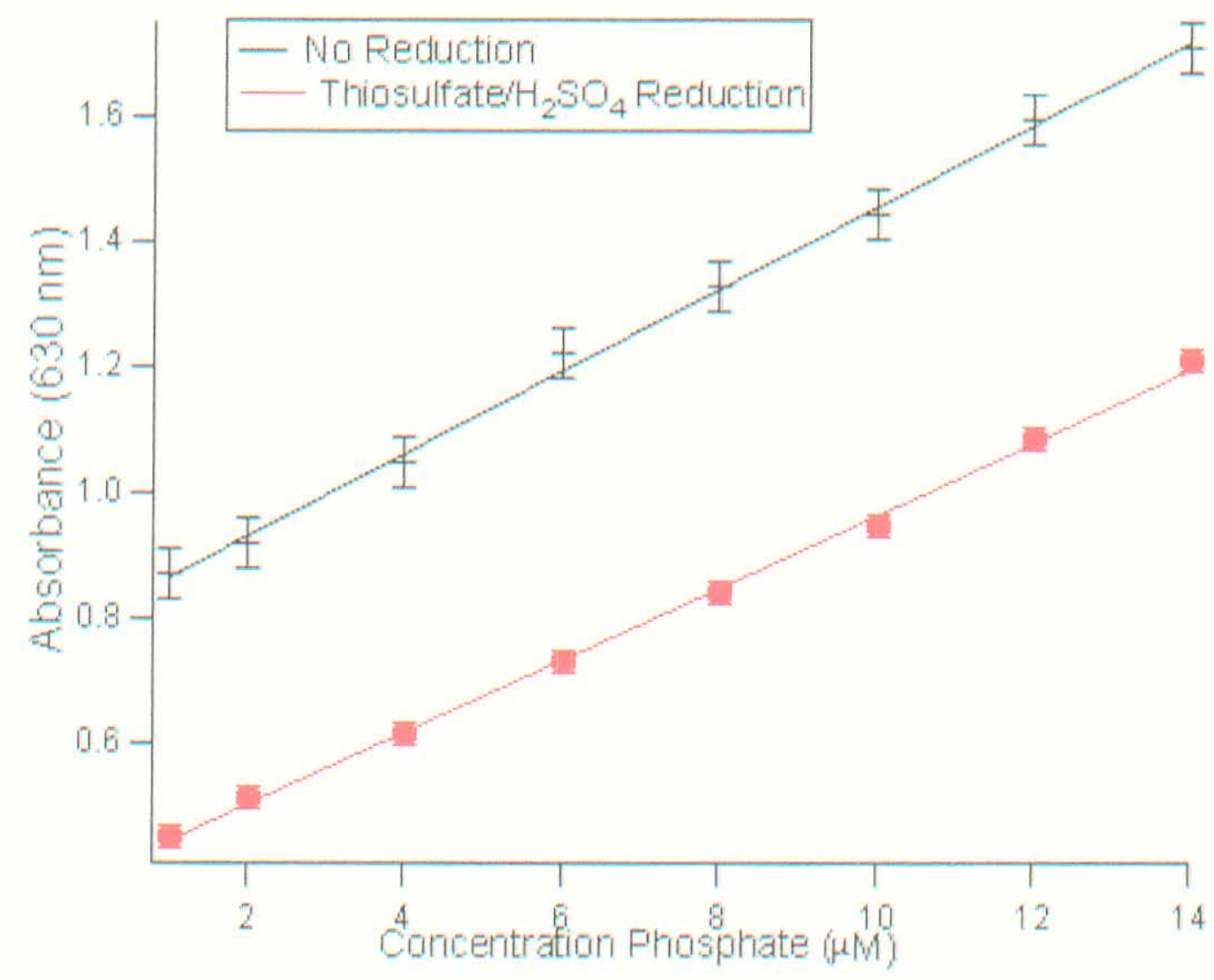

Figure 4-13: Thiosulfate pre-reduction does not lead to a decrease in sensitivity and reproducibility, or diminished correlation.

\subsection{Comparison of Reducing Agent Efficacy}

Both thiosulfate and L-Cysteine showed great promise as reducing agents for As(V) in initial studies. Before utilizing either reduction method on soil samples from Loudon County, additional studies were conducted on solutions containing both 
phosphate and arsenate. Using solutions with known concentrations of phosphate and As(V) allowed for a more accurate determination reducing agent efficacy.

Standard samples were created at several phosphate and arsenate concentrations and reduced using both methods. To control for errors resulting from inaccuracies in the phosphate solution, phosphate only samples were developed before additional arsenate was added. The samples were analyzed and the phosphate concentration was calculated using the calibration coordinating to each method (Table 4-2).

The addition of $\mathrm{As}(\mathrm{V})$ to solutions containing phosphate caused a positive bias in calculated phosphate concentration. The $5 \mu \mathrm{M} \mathrm{As}(\mathrm{V})$ caused an additive increase in the phosphate concentration. The addition of $10 \mu \mathrm{M} \mathrm{As}(\mathrm{V})$ resulted in lower than expected calculated phosphate concentration. However, the absorbance measurements of the 10 $\mu \mathrm{M}$ solutions neared the upper limit of detection, which is likely the result of approaching the upper limit of the detection range. Phosphate solutions with concentrations greater than $18 \mu \mathrm{M}$ do not show increased absorbance coefficients that follow the linear trend. Instead the coefficients reach an asymptote and the accurate concentration cannot be calculated. Addition of $10 \mu \mathrm{M} \mathrm{As}(\mathrm{V})$ to $10 \mu \mathrm{M}$ phosphate does not produce an additive increase because it is above the upper limit of detection.

Regardless of the concentration of $\mathrm{As}(\mathrm{V})$ added, the interference was successfully eliminated using both thiosulfate and L-Cysteine. Solutions that did not have interference from $\mathrm{As}(\mathrm{V})$ still produced accurate concentrations of phosphate even after pre-reduction. Both reducing agents produced accurate calculated phosphate concentrations without introducing high degrees of uncertainty and are therefore highly compatible with the three-minute malachite green analysis. 


\begin{tabular}{|c|c|c|c|}
\hline $\begin{array}{c}{[\mathrm{As}(\mathrm{V})]} \\
(\mu \mathrm{V})\end{array}$ & $\begin{array}{l}{\left[\mathrm{PO}_{4}\right] \text { (uM) }} \\
\text { Unreduced }\end{array}$ & $\begin{array}{l}{\left[\mathrm{PO}_{4}\right](\mu \mathrm{M})} \\
\text { Thiosulfate }\end{array}$ & $\begin{array}{l}{\left[\mathrm{PO}_{4}\right](\mu \mathrm{M})} \\
\text { L-Cysteine }\end{array}$ \\
\hline \multicolumn{4}{|c|}{$\left[\mathrm{PO}_{4}\right]=0 \mu \mathrm{Y}$} \\
\hline $0^{4}$ & $0.08+1.28$ & $0.05+0.03$ & $0.97+0.22$ \\
\hline $5^{\mathrm{t}}$ & $4.36+0.23$ & $-1.38+0.18$ & $0.67+0.10$ \\
\hline $10^{5}$ & $8.70+1.0$ & $-1.13+0.1$ & $1.21+0.56$ \\
\hline \multicolumn{4}{|c|}{$\left[P O_{4}\right]=5 \mu \mathrm{M}$} \\
\hline $0^{\mathrm{a}}$ & $6.50+0.66$ & $8.49+0.15$ & $6.70 \div 0.36$ \\
\hline $5^{b}$ & $10.15+0.13$ & $4.30+0.40$ & $5.64+0.05$ \\
\hline $10^{\mathrm{b}}$ & $13.84+0.49$ & $4.38+0.40$ & $5.53 \div 0.11$ \\
\hline \multicolumn{4}{|c|}{$\left|P O_{4}\right|=10 \mu M$} \\
\hline $\mathbf{0}^{4}$ & $10.02+0.04$ & $10.74+0.38$ & $9.58+0.58$ \\
\hline $5^{h}$ & $16.17+0.46$ & $10.18+0.05$ & $10.63+0.12$ \\
\hline $10^{b}$ & $17.09+0.31$ & $10.07 \pm 0.13$ & $10.9+0.17$ \\
\hline
\end{tabular}

Table 4-2: A comparison of the reduction efficiency of both thiosulfate and L-Cysteine shows that both reducing agents are capable of removing the interference of $\mathrm{As}(\mathrm{V})$.

\subsection{Reducing Agent Compatibility with Dual Absorbance Phosphate Analysis}

As discussed in Chapter 3, the phosphate analysis method was improved by utilizing dual wavelength absorbance measurements. As a result of the changes in data acquisition time and the addition of an absorbance measurement at $473 \mathrm{~nm}$, the compatibility of thiosulfate and L-Cysteine with the analysis method had to be reexamined.

Solutions of various phosphate concentrations were prepared and treated using the thiosulfate method. The absorbance was measured instantaneously at $473 \mathrm{~nm}$ and 630 nm. The results were compared to absorbance measurements at 3 minutes (Figure 4-14). 


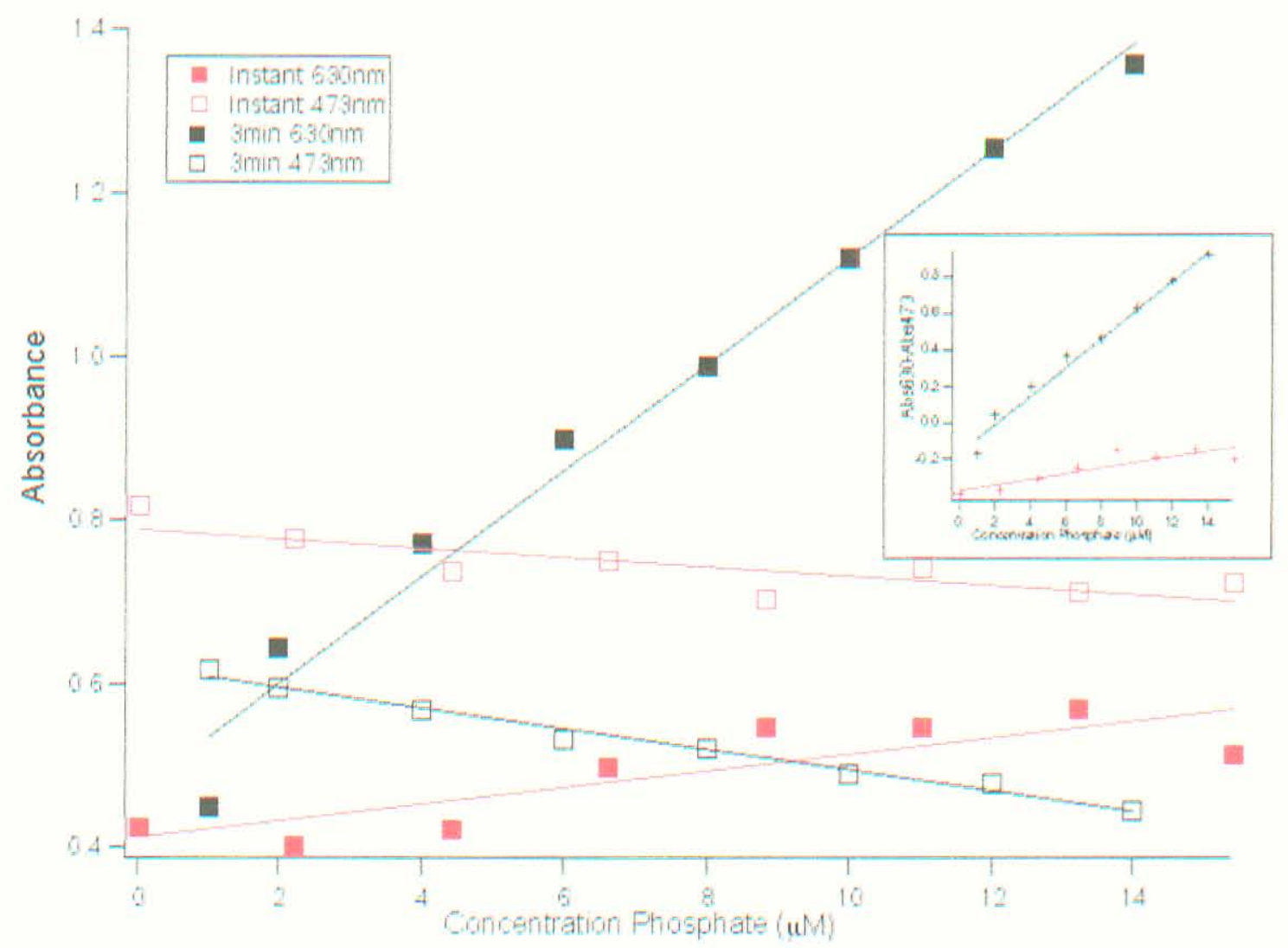

Figure 4-14: Instantaneous absorbance measurements of thiosulfate reduced solutions are significantly different from those taken after 3 minutes. The instantaneous difference calibration (inset) has a limited slope and cannot be used for phosphate calculation.

The thiosulfate treated solutions displayed dramatically different instantaneous absorbance behavior. This result was anticipated based on the kinetics of thiosulfatetreated solutions at $630 \mathrm{~nm}$. Recall that thiosulfate-treated solutions have an increase in absorbance at $630 \mathrm{~nm}$ and do not reach the maximum absorbance until after 2 minutes. Accordingly as Figure 4-14 shows, the initial absorbance at $630 \mathrm{~nm}$ is relatively constant regardless of phosphate concentration. The instant absorbance at $473 \mathrm{~nm}$ is also unchanging and thus a useful calibration cannot be created using the difference between the absorbance measurements. As a result, thiosulfate reduction cannot be used in combination with instantaneous, dual absorbance phosphate analysis. 
The experiment was repeated using the L-Cysteine method (Figure 4-15). The $630 \mathrm{~nm}$ absorbance is less sensitive to changes in phosphate concentration while the 473 $\mathrm{nm}$ absorbance is more sensitive to changes in phosphate concentration. However, the calibration created from the instantaneous difference in absorbance is nearly parallel to the instantaneous 3-minute calibration (slope: 0.0575 and 0.0667 respectively).

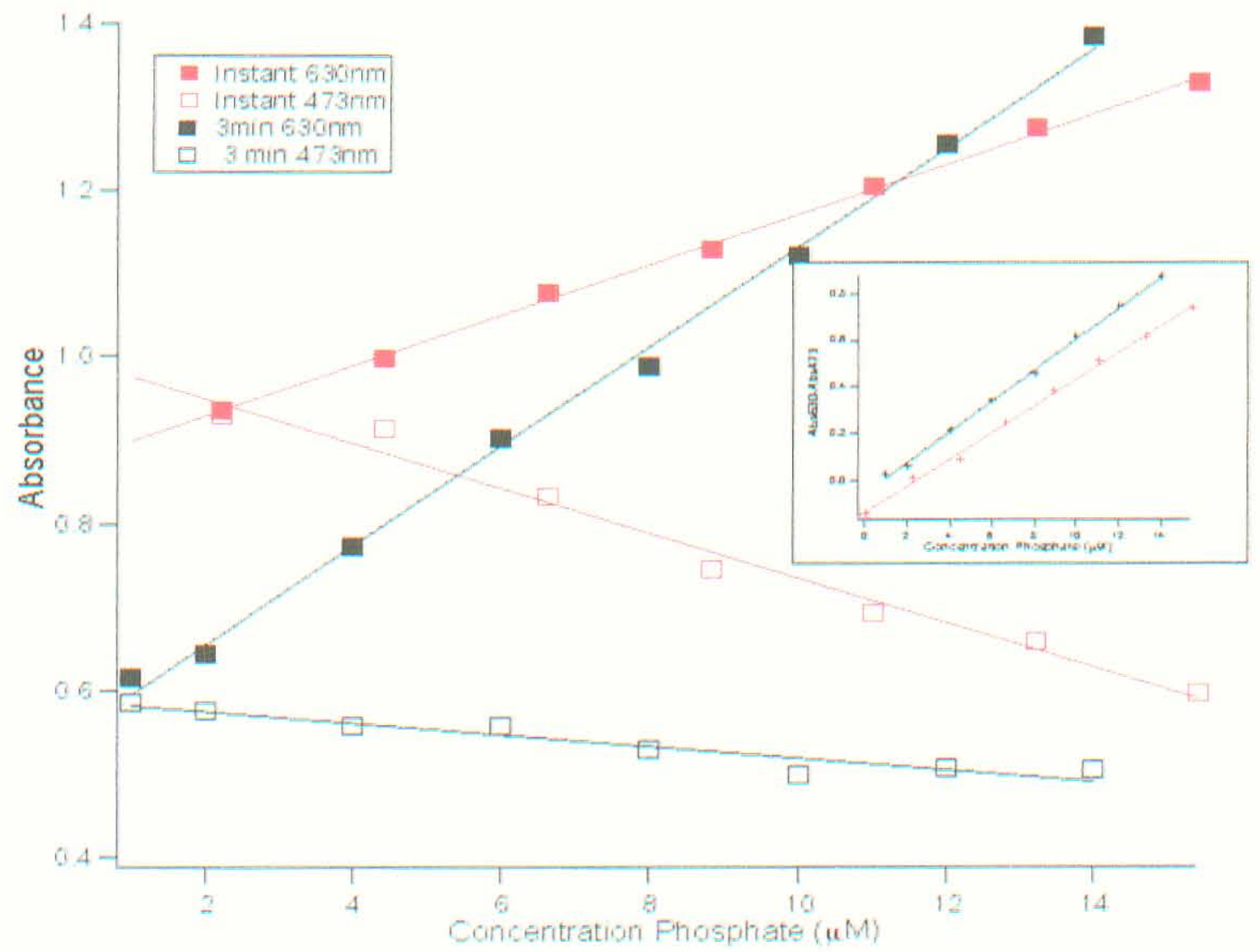

Figure 4-15: Instantaneous absorbance measurements of L-Cysteine solutions do not follow the same trends as $3 \mathrm{~min}$. absorbance. The slope of the difference calibration (Inset) created for instantaneous measurement is comparable to the slope at 3 minutes. 
In order to determine the efficacy of L-Cysteine with instantaneous measurements, solutions with known concentrations of phosphate and $\mathrm{As}(\mathrm{V})$ were reduced. The samples were analyzed in triplicate and the phosphate concentration was calculated using the calibration coordinating to each method (Table 4-3).

\begin{tabular}{ccc}
\hline $\begin{array}{c}\mathrm{As}(\mathrm{V})] \\
(\mu \mathrm{M})\end{array}$ & $\begin{array}{c}{\left[\mathrm{PO}_{4}\right](\mu \mathrm{M})} \\
\text { Unreduced }\end{array}$ & $\begin{array}{c}{\left[\mathrm{PO}_{4}\right](\mu \mathrm{M})} \\
\text { L-Cysteine }\end{array}$ \\
\hline \multicolumn{3}{c}{$\left[\mathrm{PO}_{4}\right]=0 \mu \mathrm{M}$} \\
0 & $2.0 \pm 0.5$ & $2.1 \pm 0.6$ \\
5 & $6.4 \pm 0.3$ & $1.0 \pm 1.7$ \\
10 & $8.8 \pm 0.5$ & $2.9 \pm 0.1$ \\
& & \\
& {$\left[\mathrm{PO}_{4}\right]=5 \mu \mathrm{M}$} & \\
0 & $7.1 \pm 0.1$ & $7.6 \pm 0.6$ \\
5 & $11.4 \pm 0.1$ & $6.5 \pm 0.4$ \\
10 & $14.0 \pm .2$ & $5.8 \pm 0.1$ \\
& & \\
\multicolumn{4}{c}{$\left[\mathrm{PO}_{4}\right]=10 \mu \mathrm{M}$} \\
0 & $11.2 \pm 0.1$ & $13.8 \pm 0.4$ \\
5 & $15.2 \pm 0.5$ & $12.26 \pm 0.7$ \\
10 & $18.5 \pm 0.5$ & $13.4 \pm 0.4$ \\
\hline mean \pm S.D. $(\mathrm{n}=3)$ &
\end{tabular}

Table 4-3: L-Cysteine pre-reduction does not significantly affect the accuracy or reproducibility of phosphate analysis using the instantaneous, dual absorbance method.

It is clear from Table 4-3 that the presence of $\mathrm{As}(\mathrm{V})$ diminishes the accuracy of phosphate analysis using the instantaneous, dual absorbance method The instantaneous calculated phosphate concentrations are similar to the concentrations calculated after 3minutes. For example, the calculated phosphate is approximately $5 \mu \mathrm{M}$ higher than expected in phosphate solutions containing $5 \mu \mathrm{M} A s(V)$. This behavior is identical to three minute development, which implies the change in data acquisition time did not diminish the interference of $\mathrm{As}(\mathrm{V})$. 
Pre-reduction with L-Cysteine adequately reduced $\mathrm{As}(\mathrm{V})$ to $\mathrm{As}(\mathrm{III})$ and yielded a more accurate phosphate analysis. The instantaneous phosphate concentrations are less accurate than those calculated after 3 minutes. However, the resulting data is suitable for archaeological work and L-Cysteine can be used with the instantaneous development method.

\subsection{Conclusions}

Thiosulfate and I-Cysteine are suitable reagents for the reduction of $\mathrm{As}(\mathrm{V})$ in a laboratory setting. Both display excellent compatibility with 3-minute malachite green analysis. 1,-Cysteine is a non-optimal reagent for in-stride analysis. The 20 minute reduction time at room temperature - though an improvement upon existing procedures - is not conducive to a large-scale field survey. Further kinetic studies may provide insight to a decrease in L-Cysteine reduction time.

The limited reduction time and stable reagents of the thiosulfate method are ideal for large scale, remote field surveys. However, this method is not compatible with instantaneous malachite green analysis. The 8 minute reduction and phosphate analysis is still significantly faster than any spectrophotometric phosphate analysis method previously published. Additional kinetic studies of the thiosulfate and malachite green may lead to an analysis time under 3 minutes. 


\section{SOIL ANALYSIS}

\subsection{Introduction}

While the initial laboratory studies concerning phosphate extraction and analysis yielded positive results, the ultimate goal is to produce methods that are field viable. The adapted soil preparation and extraction methods and the three minute analysis procedure were incorporated into the University of Louisville and Millsaps College "Field methods in Archaeology" course. This hands-on course allows students to learn archaeological methods at a field school conducted in Loudoun County, Virginia and provided an excellent opportunity for effectiveness of our methods to be examined.

The field school took place at the Blue Ridge Center for Environmental Stewardship (BRCES) preservation land trust in the Waters Community in Loudoun County, Virginia (Figure 5-1). 

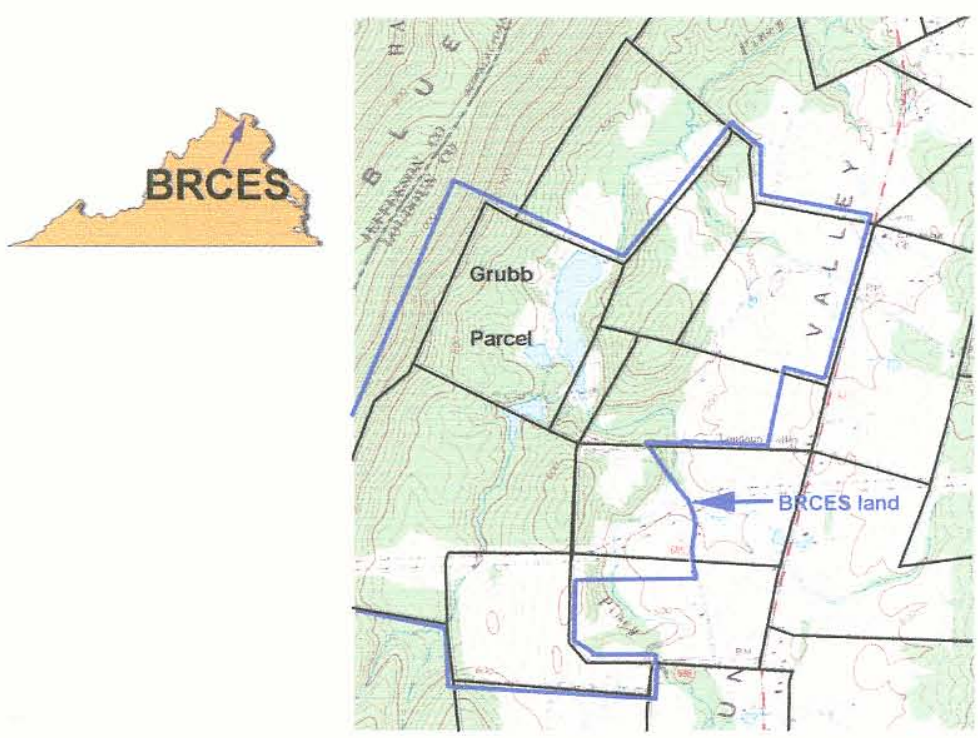

Figure 5-1: The Blue Ridge Center for Environmental Stewardship located in Loudoun Co. Virginia. Current BRCES boundaries are shown in blue and $19^{\text {th }}$ century boundaries of land parcels are shown in black.

The 364 hectares of land have been sanctioned for biological and archaeological investigation. The land has a rich history, as it was originally a settlement site for eighteenth-century European settlers. The land was owned by the Fairfax family and was leased as independent farmsteads. An "open-country" neighborhood was established containing farmsteads from as early as 1740 . Later, in the nineteenth-century, the Fairfax family sold the land as ten separate parcels. The land was subdivided at a later date, and records indicate the community may have contained twenty or more families. Of the original structures, the remains of only six homes have been located (Rypkema et al. 2007).

The identification of the additional sites has been difficult for several reasons. Much of the land is now densely forested because only one parcel has been actively farmed in the last thirty years. Satellite photographs, often employed by archaeologists in survey of large areas, cannot be used to search for signs of prior habitation due to the 
cover provided by forestation. The heavy forestation also makes the traditional "shovel testing" - in which holes are dug in 15-50 m intervals to search for artifacts- difficult, but not impossible. However, open country settlements of this time period are not known to leave a dense or wide population of artifacts. Shovel tests cover only a tiny percentage of a large site and examine small areas, and the probability of finding historic objects is low. For example in 2003 the Field Methods class conducted systematic shovel tests throughout a parcel of land believed to be the site of a farmstead belonging to Ebenezer Grubb. These efforts resulted in only one positive test, which produced a nail and a piece of glass.

It is likely that the process of shovel testing in survey can be aided by an in-stride phosphate analysis. Similar to a shovel test, the phosphate content of soil is analyzed in $50 \mathrm{~m}$ intervals. However unlike shovel tests, data gained from a phosphate test provide information that extends beyond the sampled soil if one considers the phosphate content of soil uniform for a set area. The phosphate analysis results in the creation of an archaeological "foot print", which can help to delineate the vast 71 hectare site in Loudon County believed to be the Grubbs parcel. The altered soil preparation and extraction methods as well as the three minute phosphate analysis procedure were incorporated into the 2005 Field Methods in Archaeology class to determine the effectiveness of a phosphate survey.

\subsection{Instrumentation and Reagents}

A sufficient amount of extracting solution and malachite green developing reagents were prepared before departing for the field school. All solutions were prepared 
as described in Chapters 2 and 3. Recall from these chapters that both extraction and analysis require small volumes of reagent per soil sample. Only $10 \mathrm{~mL}$ of extracting solution are needed for $1 \mathrm{~g}$ of soil. $0.3 \mathrm{~mL}$ of malachite green and $0.3 \mathrm{~mL}$ of sulfuric acid and molybdate solution are required for analysis. Since these volumes are minimal, it is easy to carry the necessary amount of solution for a days worth of work throughout the site.

Absorbance measurements were acquired using an Ocean Optics portable UVVIS spectrophotometer. The instrument is controlled by a Pocket PC and powered by a USB 2.0 line connected to a portable power source. The portable spectrometer has an area of $137.7 \mathrm{~cm}^{2}$ and the hand held $\mathrm{PC}$ has an area of $94.3 \mathrm{~cm}^{2}$. This compact instrument was set up with ease at each individual sampling site (Figure 5-2).

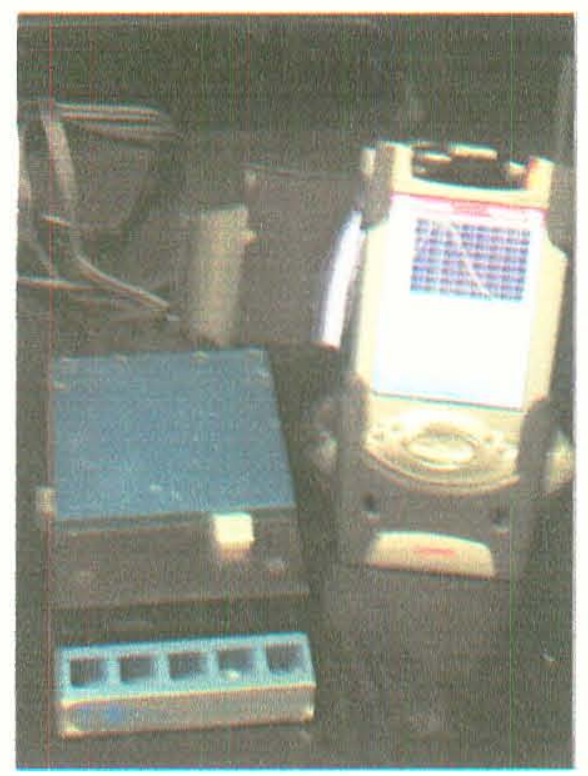

Figure 5-2 Compact and portable UV-VIS spectrophotometer used for absorbance measurements on-site. 
All the necessary glassware, reagents and tools for a days worth of sample analysis were stored in a field "toolbox" (Figure 5-3). Items in the toolbox included: mesh screen and mortar and pestle for soil preparation, syringes and filters for filtration, glass vials, developing reagents, automatic pipettes, the UV-Vis spectrometer and waste container. The toolbox was portable and easily transported among the individual sites with in the survey.

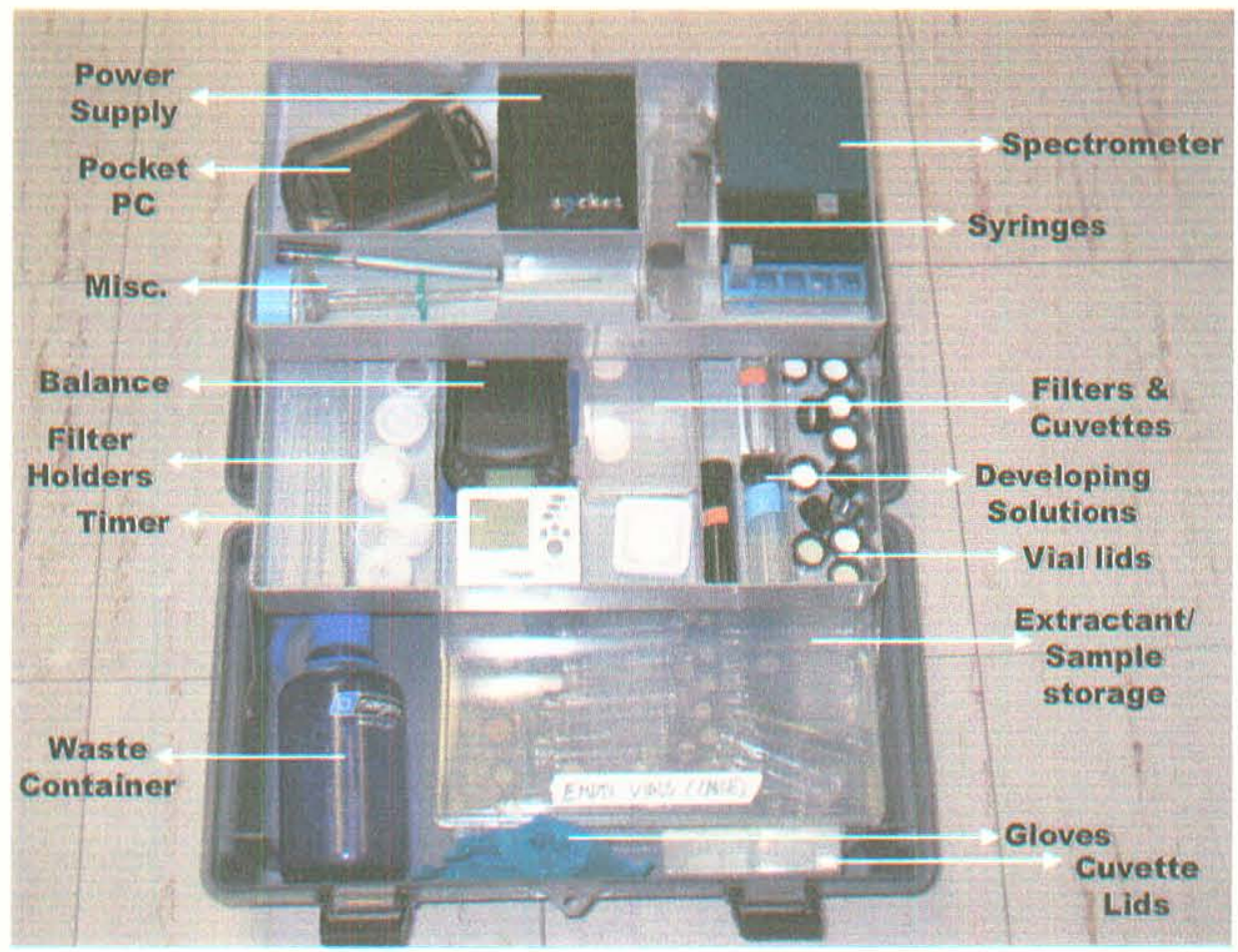

Figure 5-3: Portable toolbox used to transport all necessary items for phosphate analysis during the in-situ survey.

\subsection{In-stride Phosphate Analysis}

Shovel tests and phosphate analysis were conducted in $50 \mathrm{~m}$ intervals across the predicted Grubbs parcel and surrounding region. Soil extracted by the shovel test was sieved with $1 / 4$-inch metal screen to check for artifacts. Meanwhile, the phosphate concentrations were determined for both the A ( $6 \mathrm{~cm}$ deep) and B (12 cm deep) horizons 
of the shovel test hole. The B horizons were taken for comparison because they represented "sterile" soil unaffected by anthropogenic phosphate sources. A survey team composed of four people completed these tasks. Two people were responsible for the shovel test, one member focused on chemical analysis and the fourth member assisted wherever necessary. This procedure generated approximately 100 soil samples for phosphate analysis, and the phosphate level of each was determined before proceeding to the next location.

In order to determine areas of "high" and "low" phosphate, nine off site areas were sampled to establish baseline concentrations of phosphate in the soil. Regions of predicted low phosphate were known to have been previously uninhabited, and were generally chosen for their location, for example on a slope under heavy brush. Soils from regions known to have undergone heavy use were sampled to represent the high areas. Soil was sampled from known historic house sites the: Demory House and the Derry House. These readings served to establish the "high" marker (Table 5-1).

\begin{tabular}{ccc}
\hline ID & Site Description & $\begin{array}{c}\text { Phosphate } \\
\text { Concentration } \\
(\mu \mathrm{M})\end{array}$ \\
\hline TS01 & Sheep Pen, in use & 520.6 \\
TS02 & Demory House, side yard & 358.8 \\
TS03 & Field near office barn & 24.8 \\
TS04 & Fill from Office construction & 38.7 \\
TS05 & Young Forest on hillside, A level & 16.2 \\
TS06 & Derry House back yard & 179.4 \\
TS07 & Derry House back yard, B level & 276.7 \\
TS08 & Young Forest on hillside, A level & 134.8 \\
TS09 & Young Forest on hillside, B level & 14.0 \\
\hline
\end{tabular}

Table 5-1:Nine areas were sampled for comparison purposes. Historic house sites were used to establish "high" concentrations of phosphate. 
The establishment of high phosphate was unnecessary when comparing the all the phosphate data. When the data is considered as a whole via a contour map, areas of high phosphate can be readily identified. The accumulation of all phosphate levels permitted the creation of a gradated spot map (Figure 5- 4).

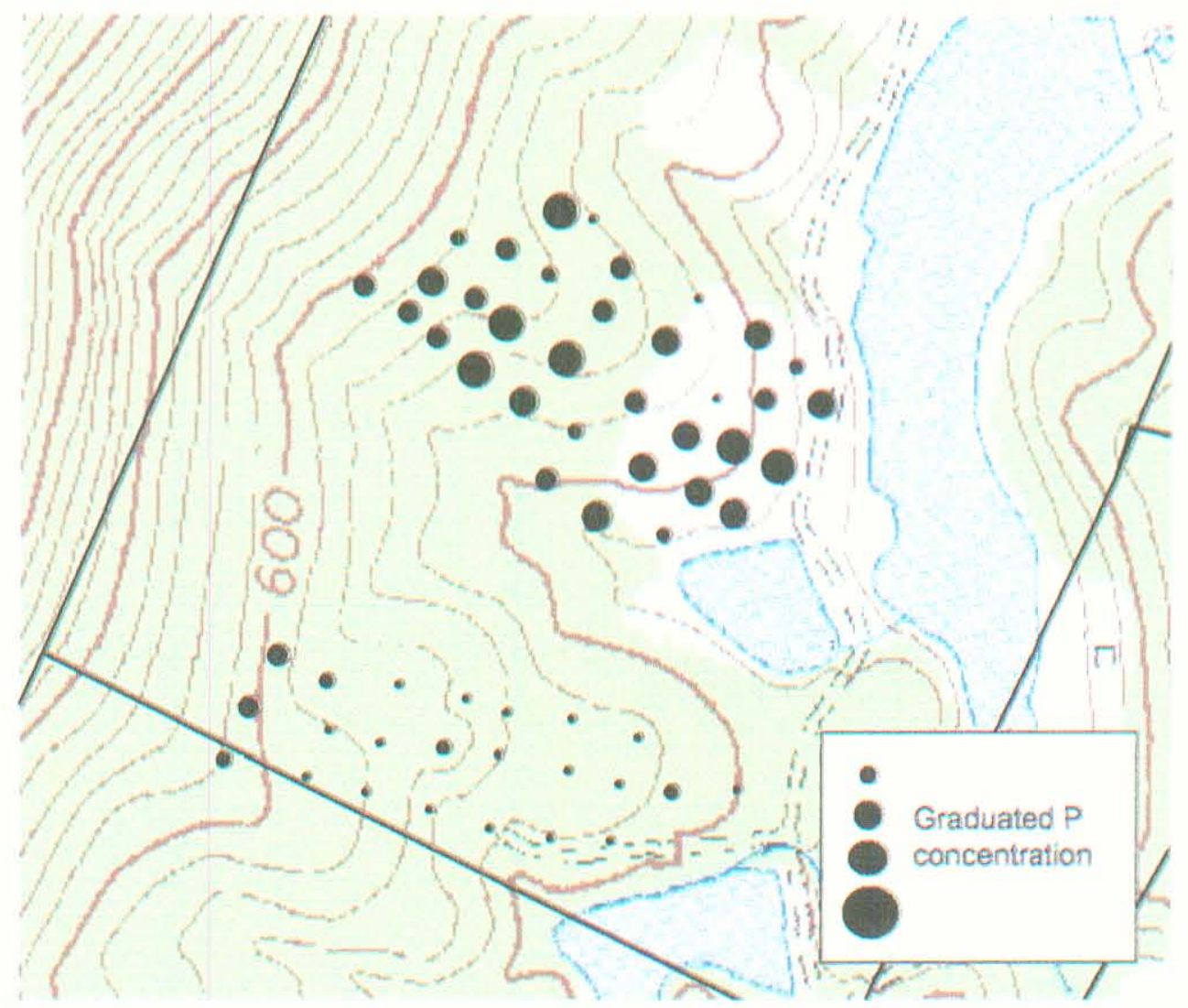

Figure 5-4: Spot map produced using phosphate concentrations determined from instride analysis. High areas in central eastern region were ignored due to modern construction interference.

In areas deemed to have a high phosphate concentration, additional shovel tests were conducted at $10 \mathrm{~m}$ intervals to refine the grid. This process produced several artifact positive shovel tests, thus validating the phosphate testing. The upper northwest quadrant of the parcel was swept with a metal detector in an attempt to locate additional artifacts that may have been missed. In the event of a positive signal from the metal 
detector, shovel tests were employed. In most cases, a nail or unidentifiable metal objects were uncovered. The coordinates of historic objects located by the metal detector were plotted on the gradated phosphate map, and the majority of the artifacts were in areas of high phosphate concentration (Figure 5-5). The correlation between phosphate and artifacts was high again confirming the validity of phosphate testing.

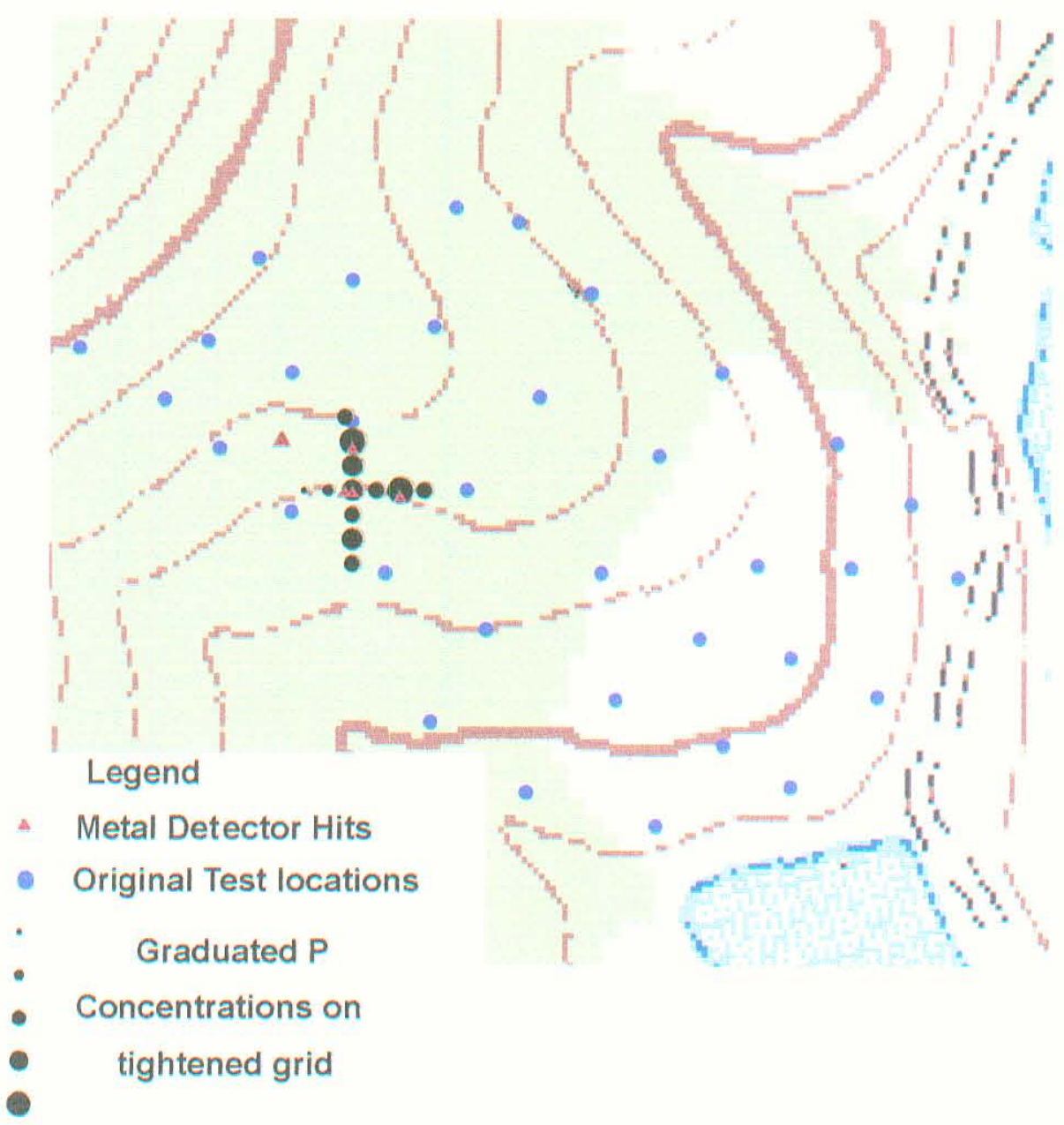

Figure 5-5: Metal detector hits producing metal artifacts (red triangle) were for the most part in areas of heightened phosphate. 


\subsection{Additional Laboratory Soil Analysis}

Additional soil was taken from each test pit to be analyzed in a traditional laboratory. Every sample was dried and analyzed in triplicate using the 3 minute malachite green method. Again, when the data are reviewed as a whole definite spikes in phosphate can be identified. It is difficult to compare the phosphate concentrations determined in the field with those determined in the laboratory because the data arises from separate soil extractions and analy sis was performed on different instruments, which have slightly different calibration curves, and the preparation is significantly more elaborate in the laboratory. However, while the actual magnitude of phosphate differs, the relative correlation remains.

During the initial phosphate survey in Loudon County, phosphate levels were determined in the field using the malachite green method and in a field laboratory using a $\mathrm{HACH}$ colorimeter for comparison. The $\mathrm{HACH}$ phosphate analysis method is typically used for analyzing water samples. However, both Terry et al. (2000) and Parnell et al. (2001) successfully utilized this portable colorometric technique for in field phosphate soil analysis. Many of the results in the field for both methods were in general agreeance. Several extracts contained an unknown interferon, which cause the sample to turn cloudy and grey. These samples could not be analyzed with HACH method.

The soil preparation and extraction process for both the malachite green and the $\mathrm{HACH}$ analysis is the same. $\mathrm{HACH}$ phosphate analysis is preformed by adding a packet of PhosVer3 powder (composed of ascorbic acid and undisclosed reagents) to $10 \mathrm{~mL}$ of soil extract. The solution must be shaken for one minute and allowed to develop for 5 minutes before analysis with the colorimeter. Six soils were selected to represent a wide 
range of phosphate concentrations. The samples were extracted and then the phosphate concentration was determined by analyzing the extracts in triplicate using both methods (Table 5-2). At low levels of phosphate both methods produce similar calculated concentrations for the representative sample. However as the concentration of phosphate increases, the $\mathrm{HACH}$ method becomes inaccurate and produces low calculated phosphate. The areas can still be identified as "high" but the large inaccuracies undermine the goals of a quantitative analysis method. This under-calculation of phosphate was also observed in the field laboratory for samples with phosphate concentrations over $300 \mu \mathrm{M}$. The inaccuracy is likely the result of difficulty in dissolving all of the PhosVer3 powder used for $\mathrm{HACH}$ analysis.

\begin{tabular}{|c|c|c|c|}
\hline Sample & $\begin{array}{c}{\left[\mathrm{PO}_{4}{ }^{3-}\right]_{\text {avg }} \pm \text { s.d. }} \\
(\mu \mathrm{M}) \\
\text { Hach }\end{array}$ & $\begin{array}{c}{\left[\mathrm{PO}_{4}{ }^{3-}\right]_{\mathrm{avg}} \pm \mathrm{s} . \mathrm{d} .} \\
(\mu \mathrm{M}) \text { Malachite } \\
\text { Green }\end{array}$ & $\begin{array}{l}\text { Difference } \\
\qquad(\mu \mathrm{M})\end{array}$ \\
\hline $469 a$ & $620 \pm 9$ & $873 \pm 36$ & 253 \\
\hline $471 \mathrm{a}$ & $447 \pm 26$ & $608 \pm 55$ & 161 \\
\hline $466 a$ & $389 \pm 30$ & $428 \pm 2$ & 39 \\
\hline $513 a$ & $123 \pm 11$ & $117 \pm 4$ & 6 \\
\hline $500 \mathrm{a}$ & $90 \pm 6$ & $104 \pm 6$ & 14 \\
\hline $492 a$ & $37 \pm 4$ & $34 \pm 1$ & 4 \\
\hline
\end{tabular}

Table 5-2: Comparison of phosphate levels determined using the commercially available $\mathrm{HACH}$ colorimeter and the malachite green method. Low levels of phosphate (under $100 \mu \mathrm{M})$ are in agreement. At high levels of phosphate, the HACH method produces inaccurate results.

$\mathrm{As}(\mathrm{V})$ interference to the malachite green analysis method was not suspect until the survey of the Grubbs parcel was complete. All A level soil samples were extracted and analyzed using the 3 minute malachite green procedure. The samples were reextracted, reduced with thiosulfate and analyzed with malachite green. It is difficult to 
determine if $\operatorname{As}(\mathrm{V})$ was present in the samples by comparing two separate extractions because of the error associated with multiple extractions (Figure 5-6). However, the graph below is significant as it shows that samples reduced with thiosulfate are still identified as "high".

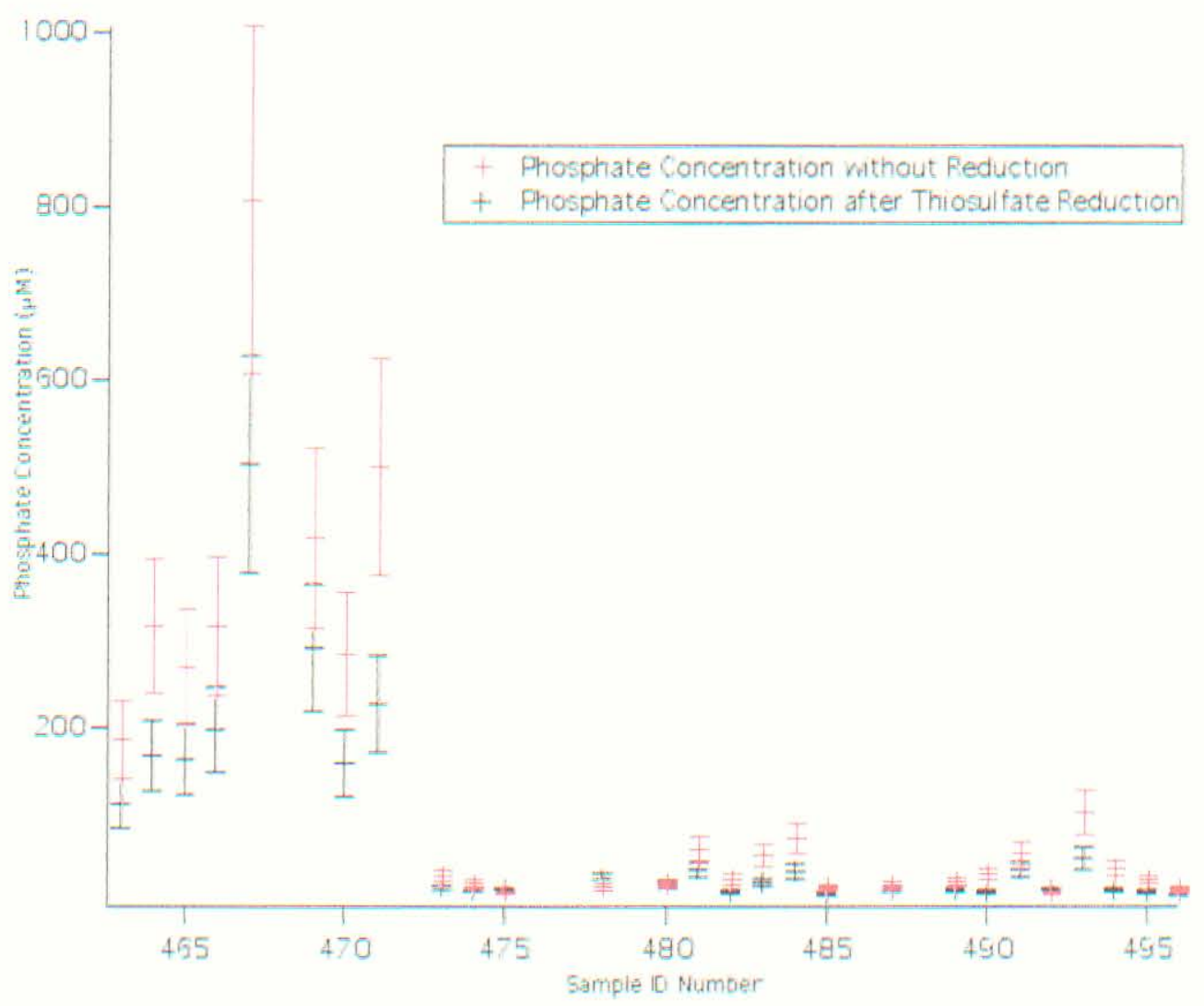

Figure 5-6: Phosphate concentrations determined after thiosulfate reduction were lower than those determined without reduction. However, it is difficult to determine if $\operatorname{As}(\mathrm{V})$ was present because data was obtained from separate extractions.

Nine soil samples were selected to represent a variety of phosphate concentrations and soil types. The soils were extracted, analyzed using the 3 minute malachite green procedure and then reduced using the L-Cysteine method. In this case, a comparison can be made with minimal error since both phosphate concentrations were determined from 
the same extracted solution (Figure 5-7). Soil samples 462a, 467a, and 471a show a significant difference between the reduced and unreduced phosphate concentrations, indicating $\operatorname{As}(\mathrm{V})$ may have been present in these particular soils. It is possible that the arsenate was present in these soils since pesticides used between 1850-1940 often contained arsenic (Fields, 1999; Robison, 2006). However overall, As(V) interference was not widespread throughout the site nor did it prevent the accurate labeling of soils as ‘high' or 'low phosphate.

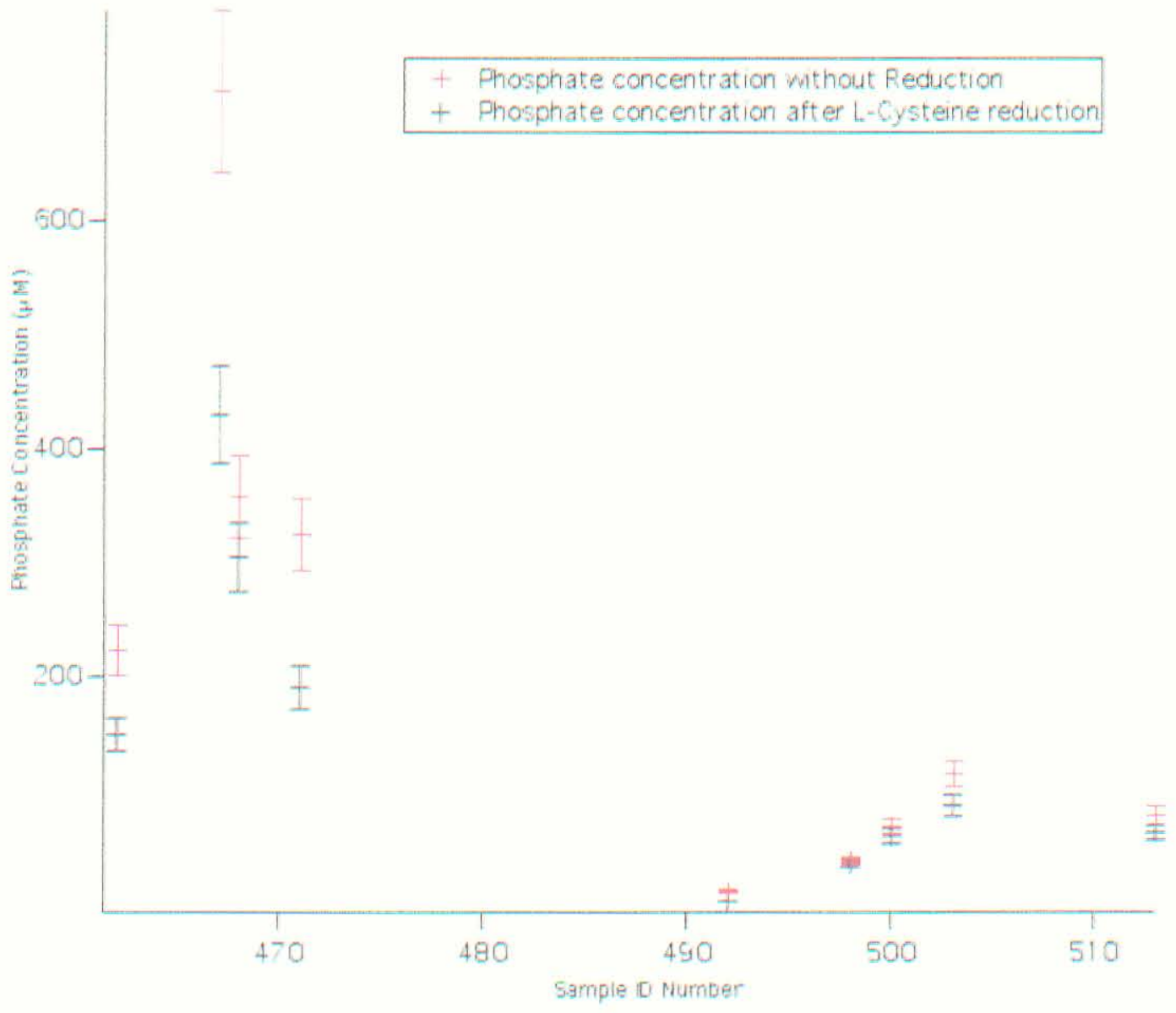

Figure 5-7: Soil reduced with L-Cysteine indicate $\mathrm{As}(\mathrm{V})$ may have been present in select soils. 
The instantaneous, dual absorbance malachite green procedure was not developed until approximately a year after the survey of the BRCES land. Several soil samples representing a range of phosphate levels were selected to be analyzed using both the three minute and instantaneous procedures. Both methods produced similar levels of phosphate without loss in precision or accuracy indicating the instantaneous method can be used with confidence in the complex soil matrix (Table 5-3).

\begin{tabular}{cccc}
\hline Sample ID & $\begin{array}{c}{\left[\mathrm{PO}_{4}{ }^{3-}\right] \pm \mathrm{S} . \mathrm{D} .} \\
(\mu \mathrm{M}) \\
\text { Instant }\end{array}$ & $\begin{array}{c}{\left[\mathrm{PO}_{4}{ }^{3-}\right] \pm \mathrm{S} . \mathrm{D} .} \\
(\mu \mathrm{M})\end{array}$ & $\begin{array}{c}\text { Difference } \\
(\mu \mathrm{M})\end{array}$ \\
\hline $466 \mathrm{a}$ & $460 \pm 5$ & $428 \pm 2$ & 32 \\
$471 \mathrm{a}$ & $621 \pm 31$ & $608 \pm 55$ & 13 \\
$492 \mathrm{a}$ & $31 \pm 2$ & $34 \pm 1$ & -2 \\
$500 \mathrm{a}$ & $96 \pm 1$ & $104 \pm 6$ & -8 \\
$513 \mathrm{a}$ & $117 \pm 8$ & $117 \pm 4$ & 0 \\
\hline
\end{tabular}

Table 5-3: Phosphate concentrations determined using the instantaneous, dual absorbance procedure do not result in a loss of accuracy or precision when compared to the three minute method.

\subsection{Conclusion}

Phosphate prospection of 71 hectares of the BRCES land proved to be a useful tool for the location of historical objects. Areas of high phosphate concentration were easily distinguished which aided in the excavation of several artifacts. The malachite green procedure is simple and can be performed by those with a limited chemical background. Training on the instrumentation and data interpretation are sufficient for those who perform the analysis. 
Clearly, the truncated analysis times of both the 3 minute and instant phosphate analysis methods are a benefit over the longer analysis times of the $\mathrm{HACH}$ colorimeter and other analysis method when conducting a dynamic phosphate survey. Additionally, the malachite green method was used successfully on all the soil samples, unlike the HACH method, which was incapable of providing data in several cases

As a result of the increased versatility of the portable spectrophotometer, the initial start up costs for the malachite green method are increased. The UV/Vis spectrometer, hand held computer, and power supply cost approximately $\$ 2,500$. The instrumentation cost represents the majority of the expense for conducting this method since the developing reagents can be obtained for approximately $\$ 150$. The $\mathrm{HACH}$ colorimeter is approximately $\$ 300$ and the continued cost of purchasing the developing packets. However, it is worth noting that the UV/Vis spectrometer provides significantly more information and can be used for many applications when compared to the colorimeter. 


\section{FUTURE DIRECTIONS AND CONCLUSIONS}

\subsection{Future Directions}

Results from the phosphate prospection of the Grubbs parcel in Loudon County, Virginia successfully helped identify the abandoned settlement areas. While phosphate analysis is undeniably a useful tool for site delineation, several additional investigations must be conducted to examine the versatility of the malachite green analysis procedure.

Consideration must be given to variations in extractable phosphorus resulting from seasonal changes. The changes in rainfall from season to season alters the soil $\mathrm{pH}$. A change in the soil $\mathrm{pH}$ results in a change in the phosphorus fixation, which in turn affects the amount of extractable phosphorus. Variations, if any, can be ignored during a short term, relative analysis. However, for long-term archaeological surveys variations would prevent the comparison of data acquired from different periods. Currently our lab is investigating this potential problem by analyzing soil samples excavated on a monthto-month basis from a remote location on the BRCES land. Preliminary results for soil sampled during October of 2006- January of 2007 show little variation in the phosphate extracted by the Mehlich3 procedure. Before definite conclusions can be drawn, results must be obtained for soil samples spanning the entire year.

The interference of $\operatorname{As}(\mathrm{V})$ was eliminated by using thiosulfate and 1 -Cysteine However other components of soil, such as silicate, are capable of interfering with 
malachite green analysis. The extent of the interface as well as methods for the elimination of the interference, must be studied in order to ensure accurate phosphate analysis.

Investigating methods for the spectrophotometric detection of additional elements with archaeological significance is by far the most important future work. Expanding the number of elements that can be analyzed fully exploits the sophistication of the portable UV/Vis spectrophotometer. Iron, for example, can easily be analyzed using thiocyanate. The spectrum of iron (III) thiocynate consists of one peak with a maximum absorbance of $450 \mathrm{~nm}$. The absorbance of FeSCN follows Beer's law for iron concentrations between $250 \mu \mathrm{M}-1.2 \mathrm{mM}$. Ten soil samples from the Grubbs parcel were analyzed to determine the iron content (Table 6-1). The soil samples were extracted using $2 \mathrm{M} \mathrm{H}_{2} \mathrm{SO}_{4}$ for thirty minutes in a steam bath. $0.6 \mathrm{~mL}$ of $60 \mathrm{mM} \mathrm{KSCN}$ in $0.1 \mathrm{M} \mathrm{HCl}$ were added to $3 \mathrm{~mL}$ of soil extract. The absorbance coeffecient was recorded immediately.

\begin{tabular}{cc}
\hline Sample ID & $\begin{array}{c}{[\mathrm{Fe}+] \pm \text { S.D. }} \\
(\mathrm{mM})\end{array}$ \\
\hline $478 \mathrm{a}$ & $12.90 \pm 0.44$ \\
$479 \mathrm{a}$ & $2.94 \pm 1.13$ \\
$480 \mathrm{a}$ & $16.02 \pm 0.76$ \\
$481 \mathrm{a}$ & $10.67 \pm 0.44$ \\
$482 \mathrm{a}$ & $10.23 \pm 0.64$ \\
$483 \mathrm{a}$ & $12.84 \pm 0.85$ \\
$484 \mathrm{a}$ & $15.31 \pm 0.84$ \\
$485 \mathrm{a}$ & $18.68 \pm 1.42$ \\
$486 \mathrm{a}$ & $9.69 \pm 0.60$ \\
$488 \mathrm{a}$ & $8.23 \pm 0.43$ \\
\hline $\mathrm{n}=5$ &
\end{tabular}

Table 6-1: Iron concentration in 10 soil samples from Loudon County, Virginia. Overall, there is little variation in the concentration of iron. 
With the exception of sample 479a, there was little variation in the iron content of these samples. Also, there was not a correlation between the iron content and the Mehlich III phosphate content of the soils (Figure 6-1).

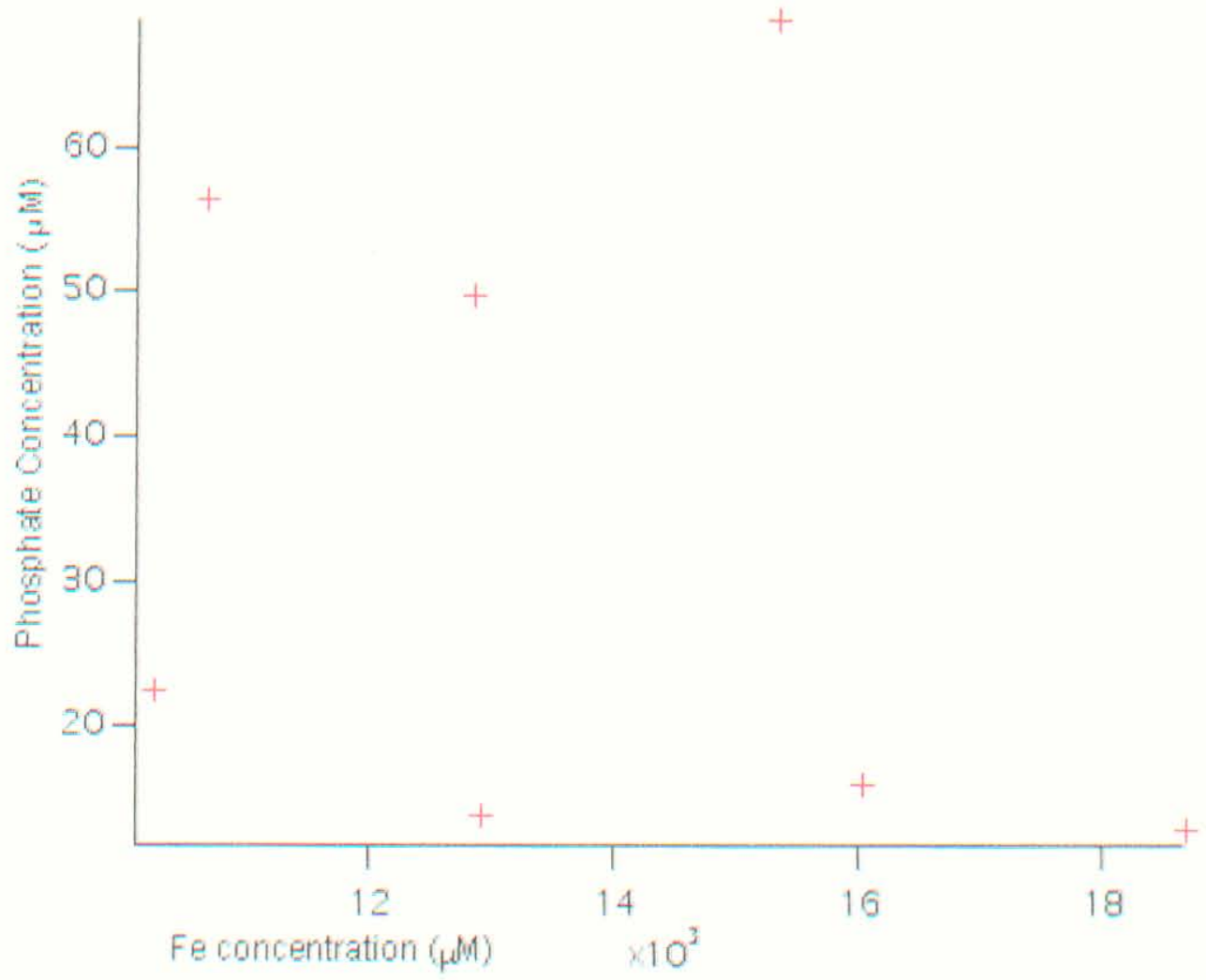

Figure 6-1: A plot of the level of iron and level of phosphate in 7 soil samples from Loudoun County, Virginia do not display a correlation.

Although valuable information was not gained by determining the iron levels in these soils, that does not eliminate the importance of iron analysis for other archaeological surveys. The minerals and elements that provide information of significance vary between civilizations. For example, Terry et al. (2000) compared the concentration of iron in specific sections of houses to the iron concentration at an off-site location at an archaeological site in Aguateca, Guatemala. The analysis reveled several hot spots of iron, which indicates the area was used for the preparation of pigments and 
dyes. Terry et al. also discovered high concentrations of iron outside of the kitchen area. The accumulation of iron is believed to be the result of guards sharpening their machetes.

Terry and coworkers are not alone in their approach of multi-elemental analysis of soil samples. Many other archaeologists have found success by identify the enrichment of elements such as lead, zinc, calcium, copper and nickel to name a few. In all cases the analysis was performed in a laboratory setting usually by means of ICP-MS. By determining the concentrations of these elements infield using UV/Vis analysis adjustments can be made to the soil sampling grid, which results in a more efficient survey. In order to actualize the goal of in stride multi-elemental analysis new extraction methods and analysis procedures must be examined and modified to meet the requirements of in situ analysis.

\subsection{Conclusions}

Archaeological survey can be slow and unsuccessful since it can be difficult to determine the areas that were once settlement sites, especially when scanning over large regions. Many anthropogenic activities lead to chemical enrichment of the soil and thus soil analysis is useful for determining site boundaries. Inorganic phosphate is an excellent indicator of prior human habitation. Unlike traditional survey methods, such as shovel testing, the information gained through phosphate prospecting extends far beyond the sample site itself. Several archaeologists have employed soil phosphate extraction procedures and quantitative phosphate analysis methods. The methods utilized were not conducive to large-scale surveys because extraction and analysis procedures were long and complex. In order to expedite the process of analyzing a multitude of soil samples, qualitative methods, such as the spot test, were employed. The qualitative analysis 
procedures are undesirable because results are often inaccurate and are subject to experimenter interpretation.

For phosphate analysis to be incorporated into a large-scale survey, the soil preparation process was shortened. Rather than allowing the soil to air dry, soil is extracted wet. While the addition of water mass introduces error to the analysis method, the inaccuracies are corrected by adjusting the phosphate calculation based on the soil water mass. An adjustment is unnecessary if the data are considered relative rather than absolute. Of course, the surveyor must remain aware of the weather conditions during the survey since large changes in rainfall affect the accuracy of analysis. Comparative analysis of all phosphate concentrations allows the sample sites to be designated as high, low, or moderate phosphate. Additional sampling is conducted in the vicinity of the high areas, thereby increasing the probability of locating areas of prior habitation.

The malachite green phosphate analysis procedure can be conducted instantaneously using the dual absorbance method or after 3 minutes if the absorbance is monitored at $630 \mathrm{~nm}$. When combined with the shortened soil preparation procedures, analysis can be completed in less than 15 minutes, which allows phosphate analysis to be incorporated into large-scale archaeological surveys.

In the presence of $A s(V)$, the malachite green analysis procedure produces inaccurate results. $\mathrm{As}(\mathrm{V})$ also forms an aggregate with a maximum absorbance at 630 $\mathrm{nm}$. Therefore the $\mathrm{As}(\mathrm{V})$ must be reduced to $\mathrm{As}(\mathrm{III})$, which is incapable of forming a complex with malachite green. Both L-Cysteine and thiosulfate are effective reducing agents for $\mathrm{As}(\mathrm{V})$. L-Cysteine is compatible with both phosphate analysis time scales, but the 20-minute reduction time is non-optimal for fieldwork. Thiosulfate requires 5 
minutes for reduction and thus the method is suitable for large-scale surveys. However, the method can only be used in combination with the 3-minute analysis as a result of changes in development stemming from alterations to solution $\mathrm{pH}$. During in-stride analysis, soil samples suspected of containing $\mathrm{As}(\mathrm{V})$ can be identified by the change in the development of malachite green. As(V) positive samples should be reduced with thiosulfate and developed using the 3-minute procedure.

The soil extraction and phosphate analysis procedure were incorporated into a large-scale survey of Loudoun county Virginia. Approximately 100 soil samples were collected and analyzed for phosphate content. Each soil was labeled as high or low phosphate helped to illuminate areas that may have been homesteads. Several objects of historical significance were excavated from areas in proximity to soil deemed high, confirming the validity of phosphate testing.

Although the procedures were successfully incorporated into the Loudoun County excavation, possible limitations can be identified. Many of the issues are inherent in all phosphate analysis methods. For example seasonal changes occurring during long-term excavations affect phosphate extraction from soil, which alter the accuracy of the assignment of high and low phosphate. A year-long study is currently in progress to examine the impact of seasonal changes on phosphate extraction. Changes in the soil type affect the extraction efficiency, and it is also difficult to assign high and low distinctions to large-scale sites with changing geographic features. In this case, the problem can be remedied by analyzing the data in sub-sections with similar soil features as opposed to examining the site as a whole. 
The portable UV/Vis spectrometer used with this method can monitor the full visible spectrum, and the potential to detect other ions is great. The soil analysis procedures can be adapted to meet the specific needs of each site by detecting other ions of significance, such as iron, to fully recognize the versatility of the instrument and soil analysis technique.

The largest drawback by far is the cost incurred with these procedures. The greatest expense is associated with the portable spectrophotometer, battery pack and the hand held computer. These expenses are one time, and the continual costs of the analysis procedures are minimal. Once the equipment has been acquired the cost per sample is significantly smaller than the price of sending out samples for external analysis, and the results can be obtained in minutes rather than weeks.

Despite this drawback, the phosphate detection method explained in this work is ideal for dynamic phosphate prospection. Large sites, which can produce over a hundred soil samples, can be analyzed on-site efficiently to produce contour maps showing the relative amounts of phosphate. The immediate results allow archaeologists to adapt the survey and gather additional data in areas of high phosphate that may have otherwise been missed using traditional techniques. Ultimately, the phosphate contour map is a tool to be combined with other archaeological methods to determine boundary lines of expansive archaeological sites and delineate the individual structures within a site. 


\section{REFERENCES}

Aggarwal, S. G., Flow Injection Analysis Spectrophotometric Speciation of Iron in Rain, Fog, Dust, and Soil Samples. Journal De Physique IV 2003, 107, 13-19.

Altmann, H. J.; Fürstenau, E.; Gielewski, A.; Scholz, L., Photometrische Bestimmung kleiner Phosphatmegen mit Malachitgrün. Z. Anal. Chem 1971, 256, 274-276.

Baykov, A. A.; Evtushenko, O. A.; Avaeva, S. M., A malachite Green Procedure for Otrhophosphate Determination and its use In Alkaline Phosphatase-Based Enzyme Immunoassy. Analytical Biochemistry 1987, 171, 266-270.

Bjelajac, V., A Validatiion Test of a Field-Based Phosphate Analysis Technique. Journal of Archaeological Science 1996, 23, 243-248.

Boampong, C.; Brindle, I. D.; Le, X.-c.; Pidwerbesky, L.; Ponzoni, C. M. C., Interference Reduction by L-cystine in the Determination of Arsenic by Hyrdide Generation. Analytical Chemistry 1988, 60, 1185-1188.

Broberg, O.; Pettersson, K., Analytical Determination of Orthophosphate in Water. Hydrobiologia 1988, (170), 45-59.

Cao, X. and L. Q. Ma; Effects of compost and phosphate on plant arsenic accumulation from soils near pressure-treated wood. Environmental Pollution 2004 (132) 435-442.

Carvalho, L. H. M.; De Koe, T.; Tavares, P. B., An Improved Molybdenum Blue Method for Simulataneous Determination of Inorganic Phosphate and Arsenate. Ecotoxicology and Environmental Restoration 1998, 1, (1), 13-19.

Cavanagh, W. G.; Hirst, S.; Litton, C. D., Soil Phosphate, Site Boundaries, and Change Point Analysis. Journal of Field Archaeology 1988, 15, (1), 67-83.

Chen, H.; Brindle, I. D.; Le, X.-c., Prereduction of Arsenic(V) to Arsenic(III), Enhancement of Signal and Reduction of Interferences by L-Cysteine in the Determination of Arsenic by Hydride Generation. Analytical Chemistry 1992, 64, 667672.

Chriswell, C. D.; Schilt, A. A., Spectropotometric Determination of Iron in Acods and acidic Solutions by Extraction-Formation Reaction Inovlving 3-(2-Pyridyl)-5,6-diphenyl1,2,4-triazine as the Chromogenic-Extraction Reagent. Analytical Chemistry 1974, 46, (8), 992-996. 
Cook, D. E.; Kovacevich, B.; Beach, T.; Bishop, R., Deciphering the Inorganic Chemical Record of Ancient Human Activity Using ICP-MS: a Reconnaissance Study of Late Classic Soil Floors at Cancuén, Guatemala. 2006.

Cornforth, I.S., "The fate of phosphate fertilizers in soil", in Chemicals and Soils, Department of Soil Science Lincoln University.

Craddock, P. T.; Gurney, D.; Pryor, F.; Hughes, M. J., The Application of Phosphate Analysis to the Location and Interpretation of Archaeological Sites. Journal of Archaeological Science 1985, 142, 361-376.

Crowther, J., Soil Phosphate Surveys: Critical Approaches to Sampling, Analysis and Interpretation. Archaeological Prospection 1997, 4, 93-102.

D'Angelo, E.; Crutchfield, J.; Vandiviere, M., Rapid, Sensitive, Microscale Determination of Phosphate in Water and Soil. Journal of Environmental Quality 2001, 30, 2206-2209.

Dasgupta, P. K.; Huang, H.; Zhang, G.; Cobb, G. P., Photometric Measurement of Trace $\mathrm{As}(\mathrm{III})$ and $\mathrm{As}(\mathrm{V})$ in Drinking Water. Talanta 2002, 58, 153-164.

Duma, G., Phosphate content of Ancient Pots as Indication of Use. Current Anthropology $1972,13,(1), 127-130$.

Eidt, R. C., Detection and Examination of Anthrosols by Phosphate Analysis. Science 1977, 197, (4311), 1327-1333.

Entwistle, J. A.; Abrahams, P. W.; Dodgshon, R. A., The Geoarchaeological Significance and spatial Variability of a Range of Physical and Chemical Soil Properties from a Former Habitation Site, Isle of Skye. Journal of Archaeological Science 2000, (27), 287303.

Frank, J. Determination of Arsenic and Arsenic Species in Ombrotrophic Peat Bogs from Finland. University Heidelberg, 2005.

Fields, T,. Findings and Recommendations for the Remediation of Historic Pesticide Contamination. H.P.C.T Force. 1999

Goulden, P. D.; Brooksbank, P., Automated Phosphate Analysis in the Presence of Arsenic. Limnology and Oceanography 1974, 19, (4), 705-707.

Hassan, F.A., Rapid quantitative determination of phosphate in archaeological sediments. Journal of Field Archaeology. 1981 8: .384-387.

Holliday, V. T.; Gartner, W. G., Methods of soil P analysis in archaeology. Journal of Archaeological Science 2007, 34, 301-333. 
Hutson, S. R.; Terry, R. E., Recovering Social and Cultural Dynamics from Plaster Floors: Chemical Analyses at Ancient Chunchucmil, Yucatan, Mexico. Journal of Archaeological Science 2006, (33), 391-404.

Ivšić, A. G.; Tamhina, B., Extraction and Formation of Iron (III) Thiocyanate Complexes Application for Spectrophotometric Determination of Iron. Croatica Chemica Acta 2003, 76, (4), 323-328.

Jiang, W. and S. Zhang, Adsorption of arsenate on soils Part 1: Laboratory batch experiments using 16 Chinese soils with different physicochemical properties. Environmental Pollution 2005, 138, 278-284.

Johnson, D. L., Simultaneous Determination of Arsenate and Phosphate in Natural Waters. Environmental Science and Technology 1971, 5, (5), 411-414.

Jones, J. B. J., Laboratory Guide for Conducting Soil Tests and Plant Analysis. CRC Press: New York, 2001.

Karadjova, I. B.; Lampugnani, L.; Onor, M.; D'Ulvio, A.; Tsalev, D. L., Continuous Flow Hydride Generation-Atomic Fluorescence Spectrometric Determination and Speciation of Arsenic in Wine. Spectrochimica Acta Part B 2005, 60, 816-823.

Kawakubo, S.; Naito, A.; Fujihara, A.; Iwatsuki, M., Field Determination of Trace Iron in Fresh Water Samples by Visual Spectrophotometric Methods. Analytical Sciences 2004, 20, 1159-1163.

Kopáček, J.; Borovec, J.; Hejzlar, J.; Porcal, P., Spectrophotometric Determination of Iron, Aluminum, and Phosphorus in Soil and Sediment Extracts After Their Nitric and Perchloric Acid Digestion. Communications in Soil Science and Plant Analysis 2001, 32, (9\&10), 1431-1443.

Lenoble, V.; Delachat, V.; Serpaud, B.; Bollinger, J.-C., Arsenite Oxidation and Arsenate Determination by the Molybdene Blue Method. Talanta 2003, 61, 267-276.

Lillios, K. T., Phosphate Fractionation of soils at Agroal, Portugal. American Antiquity 1992, 57, (3), 495-506.

Linge, K. L.; Oldham, C. E., Interference from Arsenate when Determining Phosphate by Malachite Green Spectrophotometric Method. Analytica Chimica Acta 2001, 450, 247252.

Maiti, D. and D.K. Das, Evaluation of different analytical methods for the estimation of available N, P, K and Zn in soil._Archives of Agronomy and Soil Science. 2007, 53, (1), 89-94. 
Manning, B. A.; Hunt, M. L.; Amrhein, C.; Yarmoff, J. A., Arsenic (III) and Arsenic(V) Reactions with Zerovalent Iron Corrosion Products. Environmental Science and Technology 2002, 36, (24), 5455-5461.

Mas, F.; Estela, J. M.; Cerda, V., Determination of Phosphate in Waters by Flow Injection Analysis. Water, Air, and Soil Pollution 1990, 52, 359-368.

Matera, V. and I. L. Hecho, A methodological approach for the identification of arsenic bearing phases in polluted soils. Environmental Pollution 2005, 126, 51-64.

Matsubara, C.; Hosaka, T.; Takai, A.; Kazuhiro, Y.; Takamura, K., Determination of Traces of Arsenate in Medicines and Chemicals after Collection by Membrane Filtration. Nippon Kagaku Kaishi 1990, 3, 267-70.

Matsubara, C.; Kikuchi, N.; Denpouya, i.; Takamura, K., A New Preconcentration Method of Hydrophobic Substances in Water Using Polyvinylmethylether as a Thermoresponsive Polymer. Aplication to Spectrophotometric Determination of a Trace Amount of Phosphate in Natural Water. Chemistry Letters 1993, 849-850.

Matsubara, C.; Yamamoto, Y.; Takamura, K., Rapid Determination of Trace Amounts of Phosphate and Arsenate in Water by Spectrophotometric Detection of their heteropoly Acid-Malachite Green Aggregates Following Pre-concentration by Membrane Filtration. Analyst 1987, 112, 1257-1230.

Mehlich, A., Mehlich 3 soil test extractant: Amodifaicaton of the Mehkich 2 extractant. . Communications in Soil Science and Plant Analysis 1984, 15, (12), 1409-1416.

Middleton, W. D., Identifying Chemical Activity Residues on Prehistoric House Floors: A Methodology and Rationale for Multi-elemental Characterization of a Mild Acid Extract of Anthropogenic Sediments. Archaeometry 2004, 46, (1), 47-65.

Motomizu, S.; Oshima, M.; Hirashima, A., Spectrophotometric Determination of Phosphorus River Water Based on the Reaction of Vanadomybdophosphate with Malachite Green. Analytica Chimica Acta 1988, 211, 119-127.

Motomizu, S.; Wakimoto, T.; Toei, K., Spectrophotometric Determination of Phosphorus and Arsenic in Stell by solvent Extraction of their Heteropolyacids with Ethyl Violet. Analyst 1983, 108, 944-851.

Murphy, J. and J.P. Riley, A modified single solution method for the determination of phosphate in natural waters. Analytica Chimica Acta. 1962, 27, 31-36.

Newton, K. and D. Amarasiriwardena, Distribution of soil arsenic species, lead and arsenic bound to humic acid molar mass fractions in a contaminated apple orchard. Environmental Pollution 2006 143, 197-205. 
Ng, J.; Gomez-Caminero, A.; Howe, P.; Hughes, M. J.; Lewis, D. R.; Moore, M.; Aito, A.: Becking, g., Arsenic and Arsenic Compounds. In World Health Organization International Labour Organization United nations Environment Programme: 2001.

Ohno. T.; Zibilske, L. M., Determination of Low Concentrations of Phosphorus in Soil Extracts Using Malachite Green. Soil Sci. Soc. am J. 1991, 55, (892-895).

Olsen. S. R.; Sommers, L. E., Phosphorus. In Methods of Soil Analysis Part 2. Chemical and Microbiological Properties, 2 ed.; ASA-SSA: Madison, 1982; pp 403-430.

Parnell. J. J.; Terry, R. E.; Golden, C., Using In-Field Phosphate Testing to Rapidly Identify Middens at Piedras Negras, Guatemala. Geoarchaeology 2001, 16, (8), 855-871.

Peters, C. A.; French, C. L., A Study of the Ferric Thiocyanate Reaction. Industrial and Engineering Chemistry 1941, 13, (9), 604-607.

Petterson. A. K.; Karlberg, B., Simultaneous Determination of Orthophosphate and Arsenate Based on Multi-way Spectroscopic-kinetic Data Evaluation. Analytica Chimica Acta 1997, 354, 241-248.

Pillai, A.; Sunita, G.; Gupta, V. K., A New System for the Spectrophotometric Determination of Arsenic in Environmental and biological Samples. Analytica Chimica Acta 2000, 408, 111-115.

Rao. A.S., K.S. Reddy, and P.N. Takkar, Malachite green method compared to ascorbic acid for estimating small amounts of phosphorus in water, $0.01 \mathrm{M}$ calcium chorite and Olsen soil extracts. Communications in Soil Science and Plant Analysis 1997, 28, 589 . 601.

Robison. G.R. and J.D. Ayotte, The influence of geology and land use on arsenic in stream sediments and ground waters in New England, USA. Applied Geochemistry 2006, $21(9), 1482-1497$.

Rypkema, Heather; Lee, Wayne; Galaty, M.; Haws, Jonthan. Rapid in-stride soil phosphate measurement in archaeological survey: A new method tested in Ioudoun County, Virginia._Journal of Archaeological Science.2007, 34, 1859-1867.

Sanchez, A.; Canabate, M. L.; Lizcano, R., Phosphorus Analysis at Archacological Sites An Optimization of the Method and Interpretation of the Results. Archueomeny 19\%, 3., (1), 151-164.

Sarris. A.; Galaty, M. L.; Yerkes, R. W.; Parkinson, W. A.; Gyucha, A.; Billingsley, D. M.: Tate, R., Geophysical Prospection and Soil Chemistry at the Early Conper Agr

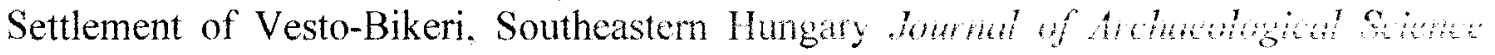
2004, 31, 972-939. 
Schlezinger, D. R., Organic Phosphorus and Elemental Ratios as Indicators of Prehistoric Human Occupation. Journal of Archaeological Science 2000, 27, 479-492.

Shi, J.-b.; Tang, Z.-y.; Jin, Z.-x.; Chi, Q.; He, b.; Jiang, G.-b., Determination of As(III) and $\mathrm{As}(\mathrm{V})$ in soils using Sequential Extraction Combined with Flow Injection Hydride Generation Atomic Fluorescence Detection. Analytica Chimica Acta 2003, 477, 139-147.

Shraim. A.; Chiswell, B.; Olszowy, Speciation of Arsenic by Hydride Generation-Atomic Absorption Spectrometry (HG-AAS) in Hydrocholoric Acid Reaction medium. Talanta $1999,50,1109-1127$.

Sinaj, S.; Stamm, C.; Toor, G. S.; Condron, L. M.; Hendry, T.; Di, H. J.; Cameron, K. C.; Frossard, E., Vadose Zone Processes and Chemical Transport. Journal of Environmental Quality 2002, 31, 319-330.

Sjobrg, A., Phosphate Analysis of Anthropic Soils. Journal of Field Archaeology 1976, 3, (4), 447-454.

Smedley, P. L.; Kinniburgh, D. G., Applied Geochemistry 2002, 17, 517-568.

Stauffer, R. E., Determination of Arsenic and phosphorus Compounds in Groundwater with Reduced Molybdenum Blue. Analytical Chemistry 1983, 55, 1205-1210.

Takamura, K., Trace Analysis Involving Preconcentration of Analyte in Solid Phases. Dojin News. 1992, 63, 3-8.

Terry, R. E.; Fernández, F. G.; Parnell, J. J.; Takeshi, I., The Story in the Floors: Chemical Signatures of Ancient and Modern Maya Activites at Aguateca, Guatemala. Journal of Archaeological Science 2004, (31), 1237-1250.

Terry, R. E.; Hardin, P. J.; Houston, S. D.; Nelson, S. D.; Jackson, M. W.; Carr, J.; Parnell, J., Quantitative Phosphorus Measurement: A Field Test Procedure for Archaeological Site Analysis at Piedras Negras, Guatemala. Geoarchaeology 2000, 15, (2), 151-166.

Todd, I. A.; Freeman, J.; Jelks, E. B.; Kent, S.; Murray, P.; Change, C.; Byrd, B. F.; Hassan, F. A., Rapid Quantitative Determination of Phosphate in Archaelogical Sediments. . Journal of Field Archaeology 1981, 8, (3), 367-390.

Tsalev, D. L.; Sperling, M.; Welz, B., Flow-injection Hydride Generation Atomic Absorption Spectrometric Study of the Automated On-line Pre-reduction of Arsenate, Methylarsonate. 2000.

Van Veldhoven, P. P.; Mannaerts, G. P., Inorganic and Organic Phosphate measurements in the Nanomolar Range. Analytical Biochemistry 1987, 161, 45-48. 
White. E. M., Cautionary Note on Soil Phosphate Data Interpretation for Archaeology. American Antiquity 1978, 43, (3), 507-508

Wilson, C. A.; Davidson, D. A.; Cressor, M. S., An Evaluation of Multielement Analysis of Historic Soil Contamination to Differentiate Space Use and Former Function in and Around Abandoned Farms. The Holocene 2005, 15, (7), 1094-1099.

Woods, W. I., The Quantitative Analysis of Soil Phosphate. American Antiquity 1977, 42 , (2), 248-252. 


\title{
FRICULUM VITAE
}

\author{
I gura Anne DeNeve \\ 4202 Lighthouse Cove \\ Jeffersonville IN 47130
}

Education: University of Louisville

Louisville, $\mathrm{KY}$

M.S. in Chemistry

Devember 2007

GPA: 3.3

Bellarmine University

Louisville, $\mathrm{KY}$

B.A. in Chemistry

May. 2004

GPA: 3.5 (Cum Laude)

Research: University of Louisville

Louisville, $\mathrm{KY}$

Soil Phosphate Analysis

January, 2005-present

Elimination of Arsenate Interference

December, 2005-present

Soil Iron Analysis

June, 2006 - July, 2006

Bellarmine University

June, 2003 - May, 2004

Work

Preparation and Characterization of Tripeptides

Experience: Sud-Chemie Inc.

Research and Development: Chemist I

Louisville, $\mathrm{KY}$

July, 2007-Present

University of Louisville

Louisville, $\mathrm{KY}$

Groundwork Education in Math and Science Fellow

July, 2005-May, 2007

University of Louisville

Louisville, $\mathrm{KY}$

Graduate Teaching Assistant

August, 2004-June, 2005

Bellarmine University

Chemistry Stock Room Assistant

Louisville, $\mathrm{KY}$

Aug., 2003-May,2004

Manuscripts: University of Louisville

Elimination of Arsenate Interference in Malachite Green Method

In preparation for the Detection of Phosphate in Archaeological Soil Samples

Posters and

Seminars:

National Science Foundation GK-12 National Meeting

March, 2007

Poster: Elimination of Arsenate Interference in Malachite Green

Method for the Detection of Phosphate in Archaeological Soil Samples 
Poster: GEMS Partnerships Established, Expanding. Envisioned:

Redefining Traditional Roles in K-12 and Graduate STEM Education

National Association for Science Teacher Education

International Meeting

Seminar: Change in Fellows' Beliefs, Attitudes, and Self-Efficacy and Elementary Teachers' Content Knowledge as a Result of Collaborative Experiences in an Urban Elementary School Setting

\section{Professional}

Memberships:

- American Chemical Society

- American Association for the Advancement of Science

- Association for Science Teacher Education 